Current Problems in Surgery ${ }^{\circ}$

\title{
The Diagnosis and Treatment of Posttransplant Lymphoproliferative Disorders
}

Michael A. Nalesnik, M.D.

Leonard Makowka, M.D., Ph.D.

Thomas E. Starzl, M.D., Ph.D.

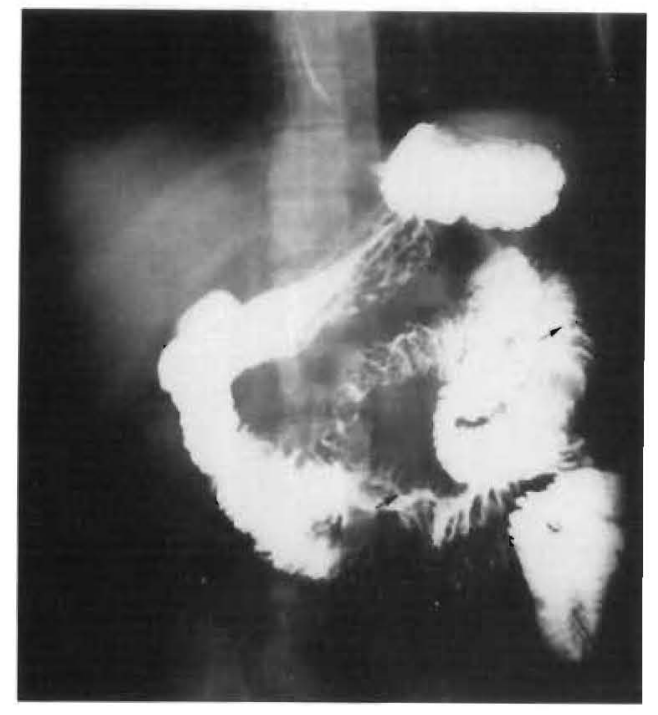

Editor-in-Chief: Mark M. Ravitch Associate Editor: Felicien M. Steichen

Editorial Board: W. Gerald Austen, Erlc W. Fonkalsrud,

Hiram C. Polk, Jr., H. William Scott, Jr., Samuel A. Wells, Jr.

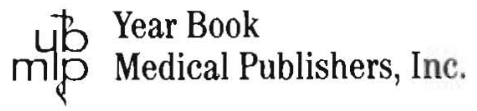




\section{Current Problems in Surgery}

MARK M. RAVITCH, M.D., Editor-in-Chief Professor of Surgery

University of Pittsburgh, Pittsburgh

FELICIEN M. STEICHEN, M.D., Associate Editor

Surgeon-in-Chief

Lenox Hill Hospital, New York

\section{EDITORIAL BOARD}

W. GERALD AUSTEN, M.D. Chief of the General Surgical Service Massachusetts General Hospital Boston

HIRAM C. POLK, JR., M.D. Professor and Chairman Department of Surgery University of Louisville Louisville
ERIC W. FONKALSRUD, M.D.

Professor and Chief of Pediatric Surgery University of California School of Medicine Los Angeles

H. WILLIAM SCOTT, JR., M.D. Professor of Surgery Vanderbilt University School of Medicine Nashville

SAMUEL A. WELLS, JR., M.D.

Professor and Chairman

Department of Surgery

Washington University

School of Medicine

St. Louis

EDITORS EMERITI

ALAN THAL, M.D.

\section{PUBLISHER'S STAFF}

Editorial Director: Nancy E. Chorpenning

Managing Editor: Craig A. Pugh

Assistant Managing Editors: James P. Fieweger; James F. Shanahan

Editorial Coordinator: Larry W. Nelson

Assistant Director, Manuscript Services: Frances M. Perveiler

Assistant Manager, Manuscript Services: Julie DuSablon

Project Manager: Nancy C. Bake

Proofroom Supervisor: Shirley E. Taylor

Vice President, Marketing: Vincent F. Douglas

Assistant Product Manager: Susan Martinsen

Special Sales Manager: Thomas Ehrhardt

Vice President, Operations: James D. Bondy

Year Book Medical Publishers, Inc.

200 N. LaSalle St.

Chicago, Illinois 60601

CUSTOMER SERVICE: 1-800-621-9262 


\section{THE DIAGNOSIS AND TREATMENT OF POSTTRANSPLANT LYMPHOPROLIFERATIVE DISORDERS*}

\author{
Michael A. Nalesnik, M.D. \\ Department of Pathology \\ University of Pittsburgh Health \\ Center \\ University of Pittsburgh \\ Veterans Administration Medical \\ Center \\ Pittsburgh, Pennsylvania
}

\author{
Leonard Makowka, M.D., \\ Ph.D. \\ Department of Surgery \\ University of Pittsburgh Health \\ Center \\ University of Pittsburgh \\ Pittsburgh, Pennsylvania
}

Thomas E. Starzl, M.D., Ph.D.

Department of Surgery University of Pittsburgh Health Center University of Pittsburgh Veterans Administration Medical Center Pittsburgh, Pennsylvania

\footnotetext{
*Supported by Research Grants from the Veterans Administration and Project Grant No. AM-29961 from the National Institutes of Health, Bethesda, Maryland, and a Research Grant from the Pathology Education and Research Foundation, Department of Pathology, Presbyterian University Hospital, Pittsburgh, Pennsylvania.

Curr Probl Surg 25(6):365-472, 1988

(C) 1988, Year Book Medical Publishers, Inc

0011-3840/88/06-365-472-\$9.95
} 
Current Problems in Surgery (ISSN 0011-3840) is published monthly by Year Book Medical Publishers, Inc., 200 N. LaSalle St., Chicago, IL 60601. Prices for 12 issues are: U.S./CANADA individuals $\$ 55.00$, institutions $\$ 72.00$, medical student or resident physician $\$ 29.95$. (Prices quoted are in U.S. dollars. Canadian orders will be billed in Canadian funds at the approximate current exchange rate. A small additional charge will be made for postage and handling.) FOREIGN: please refer below for the distributor in your area. Single issue price is $\$ 9.95$. Second-class postage paid at Chicago, IL and additional mailing offices. POSTMASTER: Send address changes to Current Problems in Surgery, Year Book Medical Publishers, Inc., 200 N. LaSalle St., Chicago, IL 60601.

\section{COPYRIGHT 두 1988 BY YEAR BOOK MEDICAL PUBLISHERS, INC.}

All rights reserved. No part of this publication may be reproduced, stored in a retrieval system, or transmitted, in any form or by any means-electronic, mechanical, photocopying, recording, or otherwise-without prior written permission from the publisher except in cases described below.

The code at the bottom of the first page in this issue indicates the publisher's consent that copies of the article may be made for personal or internal use. This consent is given on the condition that the copier pay the stated per-copy fee through the Copyright Clearance Center, Inc. (Operations Office, 27 Congress Street, Salem, MA 01970) for copying beyond that permitted by Sections 107 or 108 of the United States Copyright Law. This consent does not extend to other kinds of copying, such as copying for general distribution, for advertising or promotional purposes, for creating new collected works, or for resale.

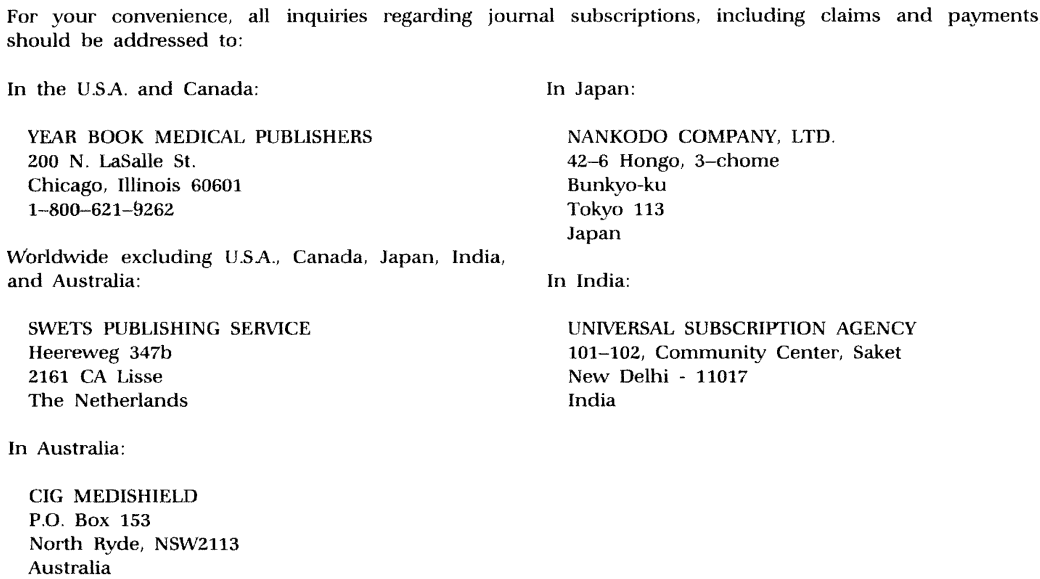

In the U.S.A. and Canada:

YEAR BOOK MEDICAL PUBLISHERS

$200 \mathrm{~N}$. LaSalle St.

Chicago, Illinois 60601

1-800-621-9262

Worldwide excluding U.SA., Canada, Japan, India and Australia:

SWETS PUBLISHING SERVICE

Heereweg $347 \mathrm{~b}$

2161 CA Lisse

The Netherlands

In Australia:

CIG MEDISHIELD

P.O. Box 153

North Ryde, NSW2113

Australia

In Japan:

NANKODO COMPANY, LTD.

42-6 Hongo, 3-chome

Bunkyo-ku

Tokyo 113

Japan

In India:

UNIVERSAL SUBSCRIPTION AGENCY 101-102, Community Center, Saket New Delhi - 11017

India

Current Problems in Surgery is listed in Index Medicus, Current Contents (CC), ISI/BioMed, and the SCI. 


\section{FOREWORD}

Dr. Thomas Starzl and his colleagues have since their Colorado days spearheaded the recognition of the occurrence of posttransplant lymphoproliferative disorders and in a series of publications have contributed to their understanding and treatment. In this issue of Current Problems in Surgery, Drs. Nalesnik, Makowka, and Starzl have produced a superb and comprehensive monograph on this subject. The article is a tour de force of combined basic and clinical sciences, together with a concrete exposition of the various clinical factors involved from etiology, recognition, and treatment to ultimate outcome. As frightening as these disorders are, the heavy price they exact still turns out to be a tolerable price for the benefits of organ transplantation in the population at risk, particularly in view of the fact, as amply shown in these pages, that with appropriate therapy the tumors may be cured or made to regress, often without sacrifice of the transplanted organ.

In what must be considered a complicated subject, much of it at the very forefront of our expanding knowledge in transplantation biology, the authors have created a lucid and readily comprehensible masterpiece. The clinical features of the disease are highlighted by case reports, each accompanied by detailed comments and appropriate illustrations.

While it is possible to operate an automobile without knowing anything about the principles of its construction, it is less satisfactory to deal with biologic problems without understanding their mechanisms and the manner in which these mechanisms are studied. The major portion of the monograph may be read by an interested physician. For those who wish to have an understanding of the basic biologic mechanisms and the scientific techniques involved, the authors have provided an appendix, a remarkably lucid exposition of the field. One suspects that this appendix in itself is destined to be a classic. It well repays reading and studying.

Mark M. Ravitch, M.D.

Editor-in-Chief 


\section{CONTENTS}

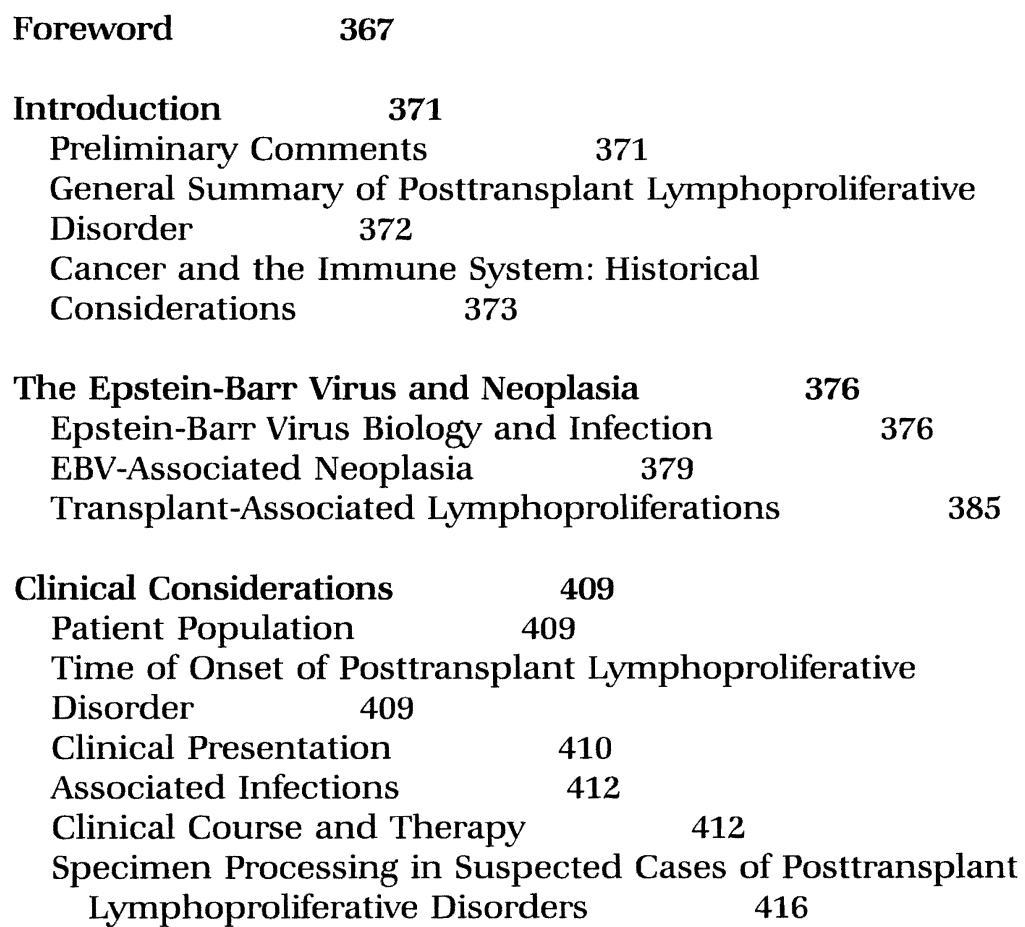

Case Presentations

417

Case Report of Kaposi's Sarcoma in a Renal Transplant

Recipient Introduction

Case Report

Comment
433

$$
433
$$

434

435

\section{Concluding Comments $\quad 436$}

Appendix: Technical Considerations Related to the Study of Posttransplant Lymphoproliferative Disorders 438 Review of Immune Interactions and Relevant Methods of Analysis 438 Consideration in the Diagnosis of Benign Versus Malignant Lymphoid Neoplasms 454 


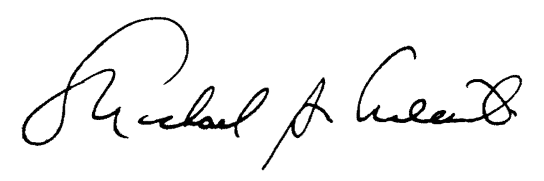

Michael A. Nalesnik, M.D., is an Assistant Professor of Pathology at the University of Pittsburgh School of Medicine and Chief of Anatomic Pathology at the Oakland Veterans' Administration Hospital. He undertook the study of transplant-associated lymphoproliferations in 1983, shortly after joining the Pathology staff at the Presbyterian University Hospital in Pittsburgh. The results of these studies reaffirmed and expanded the concept of reduced immunosuppression as a primary therapeutic maneuver in such cases. Dr. Nalesnik continues to work closely with the transplant team, providing pathologic analyses in the areas of experimental immunosuppression, organ preservation and artificial organ support.

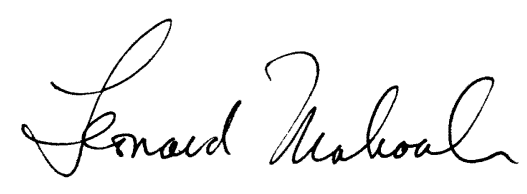

Leonard Makowka, M.D., Ph.D., is an Associate Professor of Surgery at the University of Pittsburgh School of Medicine. His clinical practice is at the Presbyterian University Hospital and at Childrens' Hospital of Pittsburgh. For some years, he has been interested in cellular growth control, particularly with reference to the hepatocyte. He has recently applied this interest to the growth characteristics of lymphoproliferative lesions that develop under the influence of immunosuppressive agents. 


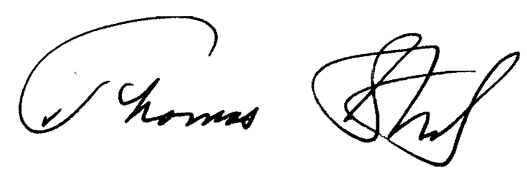

Thomas E. Starzl, M.D., Ph.D., has been a worker in transplantation since 1958. His earliest work was with experimental transplantation of the liver. He has been active in clinical renal transplantation since early 1962. In 1968, Dr. Starzl and Dr. Israel Penn described the first examples of de novo malignancies in chronically immunosuppressed patients. They noted a disproportionately large number of "reticulum cell sarcomas," now known to be $B$ cell lymphomas or lymphoproliferative disorders. As early as 1970, Dr. Starzl published evidence that these lymphomas would undergo spontaneous regression if immunosuppression was lightened, and this concept has gained both importance and credibility since then. 


\section{THE DIAGNOSIS AND TREATMENT OF POSTTRANSPLANT LYMPHOPROLIFERATIVE DISORDERS}

\section{INTRODUCTION}

PRELIMINARY COMMENTS

Posttransplant lymphoproliferative disorders (PTLDs) represent a dramatic and potentially life-threatening range of conditions which occur in approximately $2 \%$ of organ allograft recipients. ${ }^{165}$ A number of factors must intersect to cause this syndrome, and the immune system plays a key role at each step along the way. The physician caring for the patient with PTLD is witness to the end result of a number of immune system disturbances, namely, (1) a state of general immunosuppression which has been induced to permit survival of a foreign graft, but which also has caused concurrent interference with the major defense system against viral infection; (2) infection of the lymphoid system itself with the Epstein-Barr virus (EBV) in most cases; (3) proliferation of lymphoid cells, induced by the virus; (4) severe curtailment of downstream host immune control mechanisms against excessive lymphoproliferation; (5) the emergence of lymphocyte clones with distinct survival advantage, caused by stochastic events occurring during cell proliferation and leading to the formation of progressively more "malignant" clones.

This pathophysiologic gridlock is further tightened by two therapeutic considerations: (1) intensive chemotherapy in a previously immunosuppressed patient may cause the rapid onset of disseminated infection; and (2) discontinuing immunosuppression, either to reestablish host immune control mechanisms or as a prelude to chemotherapy, may lead to graft rejection.

It is obvious that manipulation of the immune system for purposes of organ transplantation must also take into account the multiple roles of this system in defense against both viral infection and tumor development. This monograph will review these various functions of the immune system and will point out how maladjustments of these interrelated functions can lead to posttransplant lymphoproliferative disorders. In summarizing our experience at the Univer- 
sity of Pittsburgh, we will show the range of proliferations that constitutes the syndrome of PTLD and we will stress the efficacy of a reduction in immunosuppression as a primary therapeutic intervention. We will also demonstrate the fatal outcome when diagnosis and therapy are delayed for whatever reason.

Throughout the course of this review references are made to recombinant DNA techniques, oncogenes, methods of clonal determination, and the cellular and humoral aspects of the immune response. A more detailed contextual discussion of these subjects is deferred to the Appendix. This will serve more effectively to direct the reader's attention to those basic concepts that lead directly to an appreciation of the clinical and therapeutic aspects of PTLD.

\section{GENERAL SUMMARY OF POSTTRANSPLANT}

\section{LYMPHOPROLIFERATIVE DISORDER}

Posttransplant lymphoproliferative disorder is used in this monograph as a generic term to describe proliferations of B-lymphocytes that occur in immunosuppressed transplant recipients ${ }^{90,244}$ Most if not all cases are associated with infection by EBV, ${ }^{121,218}$ which is the causative agent of infectious mononucleosis and a cofactor in nonHodgkin's lymphoma of the Burkitt subtype. ${ }^{31,32,81}$ Most transplant patients who are infected with this virus do not develop PTLD. ${ }^{154}$ Therefore, other cofactors must also be operative.

Infectious mononucleosis in normal individuals causes a proliferation of B-lymphocytes. ${ }^{210}$ This proliferation is controlled by multiple host defense mechanisms, especially by the proliferation and activity of cytotoxic T-lymphocytes. ${ }^{210}$ In organ transplant recipients, this Tlymphocyte defense system is suppressed to varying degrees by antirejection agents. Therefore the ability to control the B-lymphocyte proliferation may be seriously impaired.

There are several clinical categories of presentation for PTLD. ${ }^{107-}$ ${ }^{111,244}$ The patient may have an infectious mononucleosis-like syndrome, usually of exaggerated severity with respiratory compromise or cervical mass. In other cases, isolated organ dysfunction may occur due to infiltrative or tumorous PTLD. This presentation may localize to the allograft organ. The gastrointestinal tract and central nervous system are also common sites of involvement. There is some variability of localization depending upon the particular immunosuppressive regimen used. ${ }^{185}$ Finally, the patient may present with widely disseminated disease, often in association with serious infection.

Diagnosis is made by biopsy. The pathologic appearance of PTLD reflects its lymphoid origin. ${ }^{90}$ Early lesions appear reactive and show maturation of B-lymphocytes. The intense B-lymphocyte proliferation and subsequent maturation produce a mixed (polymorphic) ap- 
pearance that may be mistaken for non-Hodgkin's lymphoma. If the proliferation is allowed to continue, the likelihood of malignant transformation increases. Histology is a helpful but not absolute indicator of this change. At the time of diagnosis, serologic evidence of EBV infection should be sought and tumor tissue should be karyotyped and evaluated for clonal composition and presence of EBV genome.

Therapy is directed toward surgical relief of local complications and toward regression of tumor. ${ }^{244}$ Regression is effected by a partial or sometimes complete reduction of immunosuppression. In many cases an equilibrium can be achieved that will allow enough immune reconstitution to involute the proliferation, but not enough to reject the allograft. Control of the lymphoproliferation is of overriding concern in most cases.

The large majority of tumors fully respond to these measures. Early lesions may also respond to acyclovir therapy. ${ }^{109}$ Traditional antilymphoma therapy should be reserved for the rare case not responsive to these measures. Such nonresponsive tumors often result from a failure to recognize and treat the tumor during its earlier stages of development.

The significance of PTLD, beyond its immediate import to the afflicted patient, lies in its central position via-à-vis the processes of viral oncogenesis, immunosurveillance against neoplasia, and tumor progression in the human system.

\section{CANCER AND THE IMMUNE SYSTEM: HISTORICAL} CONSIDERATIONS

Interactions between the neoplastic process and the immune system have long been a subject of investigation and controversy. The first expression of a relationship between these two subjects is attributed by Klein ${ }^{145}$ to Paul Ehrlich:

Ich bin überzeugt, dass aberrierende Keime bei dem kolossal komplizierten Verlauf der fötalen und post-fötalen Entwicklung ausserordentlich häufig vorkommen, dass sie aber glücklicherweise bei der überwiegenden Mehrzahl der Menschen vollkommen latent bleiben, dank der Schutzvorтichtungen des Organismus. Wurden diese nucht bestehen, so könnte man vermuten, dass das Karzinom in einer geradezu ungeheuerlichen Frequenz auftreten wurde.

(I am convinced that aberrant germs appear very often during the fetal and neonatal development periods, which are extremely complicated. Fortunately, these germs remain inactive in the majority of people because of the immune system. If this self-protection did not exist, we could expect that carcinomas would appear with overwhelming frequency.)*

${ }^{*}$ From Klein G: Tumor immunology. Transplant Proc 1973; 5:31-41. Used by permission. 
Attributing a "policeman's" role to the immune system in the protection of the host against cancer development was a theme that would be iterated a half century later by Lewis Thomas. ${ }^{255 a}$ Discussing the implications of the newly relevant organ transplant rejection phenomenon, Thomas suggested:

"Is it possible . . . that the underlying mechanism brought into play was not originally intended to cope with either bacteria or grafts? . . . Perhaps, in short, the phenomenon of homograft rejection will turn out to represent a primary mechanism for natural defense against neoplasia.

. . . I have one other possibility to suggest. This concerns the fetal placenta and, perhaps, the phenomenon of parturition, which may also involve mechanisms symbolized by homograft rejection."

While the ability of the placenta to withstand immunologic rejection may indeed have implications for organ transplantation at some point in the future, it was the suggestion of a role of the immune system in cancer control that dominated investigative thought.

The concept of "immunologic surveillance" against tumors was most strongly promulgated in the writings of Burnet, ${ }^{32-35}$ who also cited Ehrlich as the source of much of his thinking. Burnet, who expounded the theory of clonal selection first proposed by Jerne in 1955, had previously and correctly championed this theory as the basis for the diversity of antibody production. Applying the concept of clonal selection to the subject of neoplasia, he suggested a competitive struggle for survival not only between the host and tumor but also among the tumor cells themselves.

In his model Burnet hypothesized that, during the course of evolutionary development, it became necessary for organisms to distinguish self from non-self. This ability conferred a survival advantage on those species able to recognize and thus to defend against parasitic life forms. However, the genetic diversity needed to generate a mechanism for the recognition of individuation introduced an element of looseness into previously restricted genetic control mechanisms. The benefit of this laxity was the development of the immune system. The price was the propensity to develop cancer, which arose from somatic mutations. The two processes developed around the same time in evolutionary terms. (We will later demonstrate how this "looseness" can be exploited to diagnose PTLDs and how it may also contribute to their pathogenesis in some cases.)

More specifically, Burnet used the term "immunologic surveillance" to refer to "the concept that a major function of the immunologic mechanisms in mammals is to recognize and eliminate foreign patterns arising in body (sic) by somatic mutation or some equivalent process. From the point of view of survival, this is important primarily as providing a means by which the appearance of malignant disease may be effectively cut short." ${ }^{36 a}$ Further, he attributed 
this role to the thymus dependent system, in light of what was known at the time regarding the immune system and its role in organ rejection phenomena.

The immune surveillance theory logically predicted that individuals with immunodeficiency would have an increased incidence of cancer. Studies of congenitally immunodeficient patients revealed just such an increased incidence of malignancy, up to $10 \%$ in some groups. ${ }^{94,95,98,136}$ In addition, an increased incidence of neoplasia was also found in immunosuppressed transplant recipients. ${ }^{186,245,246}$ However, it soon became apparent that this did not reflect an across-the-board increase in all forms of cancer ${ }^{86,125,136}$ In particular, the more common forms of neoplasia were underrepresented, and an inordinately high frequency of "lymphomas" was seen. ${ }^{188}$ This epidemiologic finding was one of several phenomena unanticipated by the immune surveillance hypothesis. The narrow range of tumors found with some regularity in immunodeficient patients led to serious doubts regarding the general application of immune surveillance to all human tumors. ${ }^{155}$

The statistics regarding the increased incidence of neoplasia were also questioned on the basis that the population of patients at risk was unknown. This particular criticism has been addressed with time. For example, one center for the reporting of these lesions was the Immunodeficiency Clinic at Memorial Sloan-Kettering Hospital, founded in 1973 by Good, a student and colleague of Thomas. (The Center moved to Mount Sinai Medical Center in 1976.) As of 1987 this center recorded 114 cases of malignancy in patients with common variable immunodeficiency. The most recent report from this center ${ }^{62}$ showed cancer in 11 of 98 patients $(11 \%)$ with common variable immunodeficiency prospectively followed for periods of 1-13 years. However, eight of the 11 cases were lymphoid in origin. Other similar studies have also found increases in gastric carcinomas in these patients. ${ }^{118,138}$ The general estimation of a $10 \%$ incidence of cancer in congenitally immunodeficient patients, such as those with Wiskott Aldrich syndrome, or ataxia telangiectasia, has also been upheld. ${ }^{194}$

A separate line of criticism against immunosurveillance concerned the development of tumors in nude mice. These congenitally athymic mice are characterized by a lack of T-lymphocyte activity. Accordingly, they should be expected to have an increased incidence of neoplasia. However, this was not the case. ${ }^{174,223}$ Additional evidence against the T-cell immune system as the executor of immune surveillance was the fact that this system in general required a certain time period to respond to an antigenic challenge and possibly did not recognize extremely low levels of antigens. Therefore, the role of the thymic dependent T-lymphocyte as the specific effector arm of immune surveillance was seriously questioned. In re- 
sponse to this, attempts were made to transfer the effector role over to other cell types, specifically macrophages or natural killer cells. ${ }^{250}$

A third logical inconsistency developed when it was discovered that, although some antigenic tumors were able to "sneak through" the immune system in experimental models, ${ }^{22,67}$ many spontaneous or induced tumors lacked strong antigenicity. ${ }^{145,148}$ It could be argued that this lack of antigenicity allowed them to subvert the surveillance mechanisms. ${ }^{223}$ However, one was also led to question the utility of an immune monitoring system based on detection of antigenic differences when the targets often did not express these antigens. ${ }^{196}$ Indeed, evidence was presented that, under certain conditions, the immune system might actually stimulate tumor growth. ${ }^{195}$

Though one can disprove the original premises of an argument, it does not necessarily follow that the conclusion is false. The undisputed fact that a select group of neoplasms emerge in the setting of an immunocompromised state may indicate that the normal host has developed a powerful defense system against a limited number of malignancies. ${ }^{200}$ This point of view was expressed by multiple investigators, in particular, the Kleins, ${ }^{140-148}$ who stressed the role of the immune response in protecting against viral oncogenesis. Even critics of the immune surveillance hypothesis as originally proposed have acknowledged the existence of an analogous system in a select population of cases. ${ }^{155,251}$ Conversely, strong proponents of immunosurveillance have agreed that the original concept of a T-cell defense against all forms of cancer is no longer tenable. ${ }^{142,148}$

It should be noted that reviews of immunodeficient or immunosuppressed populations have shown low-grade increases in tumors other than lymphomas. ${ }^{62,138,185,241}$ It is likely that the full range of immunodeficiency-associated cancers is not yet defined. In this regard transplantation offers a unique vista from which to understand and devise treatments for such disorders. The various immunosuppressive regimens necessary to sustain graft survival alter the immune system in divergent, yet reproducible, ways. By analyzing the behaviors of the consequent tumors and their response to a reversal of immunosuppression, we may learn which host factors are crucial for tumor control. This may ultimately be of use for the prevention of PTLDs, and for the treatment of tumors arising in constitutionally immunosuppressed patients.

\section{THE EPSTEIN-BARR VIRUS AND NEOPLASIA}

\section{EPSTEIN-BARR VIRUS BIOLOGY AND INFECTION}

Epstein-Barr virus is a ubiquitous virus that infects most of the world's population. From an evolutionary perspective, a symbiosis with man has developed such that infection with the virus at an 
early age is associated with no clinical manifestations. With the advent of industrial society and improved hygiene, infection is often delayed until the second or third decade. In these cases infection usually results in infectious mononucleosis. Once infected, a stable, lifelong virus carrier state develops regardless of the age at exposure. Powerful host control mechanisms that maintain this host-virus balance have only recently begun to be understood. A review of normal EBV infection and its control by the host is helpful in understanding the derangements that can lead to PTLD.

Epstein-Barr virus is a double-stranded DNA virus of the Herpesvirus family. ${ }^{173 a}$ The complete nucleotide sequence of the virus was deciphered in $1984 .{ }^{10}$ It was first isolated by Epstein and his coworkers in 1964 from specimens sent to his laboratory in Bristol, England, by Sir Dennis Burkitt, who was investigating a childhood tumor in Africa later to be known as Burkitt's lymphoma. Thus, at its very outset EBV was strongly implicated as a human tumor virus. In 1967, a laboratory technician contracted infectious mononucleosis while working with the recently discovered virus. In this manner the etiologic agent of mononucleosis was discovered. ${ }^{114}$ The virus has also been associated with nasopharyngeal carcinoma, lymphoproliferative disease in immunosuppressed humans and in some nonimmunosuppressed species of subhuman primates, ${ }^{233}$ some cases of CNS lymphomas, ${ }^{4}$ some salivary gland tumors, ${ }^{51}$ and a more recently observed disorder termed chronic infectious mononucleosis. ${ }^{131,211,248}$ Expanded numbers of EBV-containing cells have been described in patients with chronic lymphocytic leukemia ${ }^{5}$ and rheumatoid arthritis. ${ }^{258}$ The virus is not thought to be causative in these two disorders, and the significance of these associations is not known. Garden variety non-Hodgkin's B-cell lymphomas do not normally exhibit evidence of EBV involvement. ${ }^{4}$

The virus replicates in epithelial cells of the oropharynx ${ }^{236}$ and in the parotid gland ${ }^{267}$ From these sources, the circulating B-lymphocyte pool is also infected. ${ }^{268}$ The shedding of infectious virus in the saliva also accounts for the mode of transmission.

The virus enters B-lymphocytes via the complement receptor C3 ${ }^{130,179}$ and penetrates the cell within 12 hours in vitro. ${ }^{173 a}$ There are four stages to the life cycle: latent, followed by early, middle, and late replicative. All infected cells express Epstein-Barr nuclear antigens (EBNAs) of which several types are known. Concurrently, lymphocyte-determined membrane antigen (LYDMA) is produced. Early antigens (EAs) are produced during the early replicative phase and are necessary for DNA synthesis, which occurs during the middle replicative phase, or during the S-phase of the cell cycle. ${ }^{104}$ DNA replication in productively infected cells apparently utilizes viral DNA polymerase, which is sensitive to acyclovir. ${ }^{52}$ In latently infected cells acyclovir has no effect on virus replication, suggesting that that virus 
utilizes a cellular DNA polymerase ${ }^{52,237}$ Latency may be dependent on cellular as well as viral factors. ${ }^{83}$ Additional membrane antigens (MA) and viral capsid antigens (VCA) are formed during the late replicative period. The virus is often present in multiple copies. It may integrate into the host genome ${ }^{171}$ or may exist as free episomes.

In addition to viral replication, polyclonal proliferative activity is also induced within the B-lymphocyte itself ${ }^{28,29}$ In vivo, approximately $5 \%-20 \%$ of circulating B-cells are positive for EBNA during the first week of IM. ${ }^{213}$ These cells resemble plasma cells ${ }^{213}$ and have been shown to produce immunoglobulins in vitro. ${ }^{214}$ By the second week of IM, $<2 \%$ of circulating B-cells are positive for EBNA, and the majority of these infected B-cells no longer show plasmacytic differentiation. ${ }^{213}$

The host response to infection is complex, interactive, and incompletely understood. Early host response to infection includes NK cell activity and interferon production. ${ }^{133}$ Interferon production probably begins before the onset of clinical illness ${ }^{253}$ and decreases rapidly thereafter. ${ }^{25}$ An enzyme induced by interferon, $2^{\prime}, 5^{\prime}$-oligoadenylate synthetase, also decreases shortly thereafter. Levels of this enzyme have been correlated to levels of CD8 + cytotoxic/suppressor T-cells that occur early in the disease. ${ }^{25}$ NK cells have activity against productively infected cells, but show less activity against latently infected cells. In vitro, T-cell-derived factors (probably gamma interferon) delay, but do not prevent, B-cell transformation by the virus. ${ }^{210}$ The early T-lymphocyte response, referred to above, is thought to provide cytotoxic activity in a non-HLA-restricted manner and accounts for the atypical lymphocytosis of the disease. This activity is later surpassed by the emergence of a strong T-cytotoxic/suppressor (Tc/s) population ${ }^{71,140,210}$ which appears around 2 weeks after infection. ${ }^{133}$ These latter cells are directed against LYDMA, are HLArestricted $^{210}$ and are also thought to play a role in suppressing the maturation of B-cells into plasma cells. The exact contributions of cytotoxic versus suppressor activities are a matter of some debate at present. Some T-cell activity may also be stimulated by B-cell proliferation per se. This $\mathrm{T}$ response may represent an exaggerated form of physiologic control mechanisms operative in dampening a normal immune response. ${ }^{140}$ The Tc/s cells, by their cytotoxic actions, liberate EBNA from infected cells. Although EBNA production is seen 2448 hours after virus infection of B-cells in vitro, anti-EBNA antibodies are not detected until 30-50 days after the onset of illness. It is thought that this delay reflects the time necessary for the host to first destroy EBNA-containing cells, thus making this nuclear antigen accessible to the humoral immune system. ${ }^{210}$ In vivo, antibody responses are first seen against early antigens (anti-EA) and viral capsid antigen (anti-VCA). The anti-VCA antibodies are initially IgM, later superseded by IgG. Antibodies are also produced against the mem- 
brane antigen complex. Defective antibody responses to EBV infection may be seen in immunocompromised patients. ${ }^{115}$ The serologic changes of infectious mononucleosis are more fully discussed in the classic review of Henle and Henle. ${ }^{116}$

Although the infection and subsequent polyclonal B-lymphocyte proliferation are eventually brought under control by the host immune response, the infection persists indefinitely in a latent form. Recent evidence has suggested that a low-grade steady state equilibrium is established among virus replication in the oropharynx, B-cell infection, and B-cell destruction by immune effector mechanisms. ${ }^{268}$ It is hypothesized that virus replication occurs in the oropharynx, and it is at this site that B-lymphocytes are infected in low numbers. As they recirculate, a stage is reached at which LYDMA and EBNA are expressed in the infected cells. At this point they are subject to immune destruction, mainly by the cytotoxic T-lymphocytic component of the immunosurveillance system. ${ }^{210}$ It has been estimated that $0.01 \%-0.1 \%$ of circulating T-lymphocytes in serologically positive individuals have anti-LYDMA activity. It is thus argued that this immunosurveillance system alone should be capable of destroying isolated nests of latently infected cells unless they are replaced by newly infected cells. In addition, the observation that anti-EBNA levels are generally elevated at a constant level for life also suggests that a steady state has been reached whereby the destruction of small numbers of EBNA-positive infected B-lymphocytes maintains a detectable level of antibody production.

The concepts presented here are derived in large part from the recent excellent review of EBV-host interaction by Rickinson. ${ }^{210}$

\section{EBV-ASSOCIATED NEOPLASIA}

\section{Introduction}

The search for a viral origin of cancer was prompted by demonstration of similar systems in animals. However, despite the presence of some spontaneous systems of this sort operative in nature (e.g., Marek's disease in chickens) most work has been done with highly inbred animal species. Thus the applicability of these results to a highly outbred species such as man was difficult to establish.

Today of course, retroviruses are known to be able to contribute to cancer in both direct and indirect fashions. ${ }^{20,147,272}$ However, it was the discovery of the Epstein-Barr virus that marked the first solid link between a human virus and human cancer. ${ }^{81}$ Here we will consider the association of EBV in those cases of lymphoid neoplasms and disregard its role in other EBV-associated diseases such as nasopharyngeal carcinoma and undifferentiated carcinoma of the salivary glands. EBV-associated neoplasms in nontransplant populations will be discussed prior to a detailed consideration of PTLDs. 


\section{Burkitt's Lymphoma}

The discovery of Burkitt's lymphoma ${ }^{32}$ is a tribute to the perspicacity and tenaciousness of Sir Denis Burkitt. At the time a surgeon at the Mulago Hospital in Uganda, he noted the prevalence of jaw tumors in children coming to his clinic. Frequently these tumors were seen in association with other concurrent neoplasms. Depending upon which specialist examined a given child, a different diagnosis was reached in each case. Burkitt realized that this fragmented approach could not decipher the true origin of these neoplasms, which appeared to constitute a distinct entity. ${ }^{31}$ When he discovered that the children were all from a geographically distinct area, he applied for, and received, two research grants totalling $\$ \mathbf{7 5 . 0 0}$. With these funds he began his now famous epidemiologic studies that demonstrated the concordance in the distributions of Burkitt's lymphoma and hyperendemic or holoendemic malaria. In the course of these investigations, the Epstein-Barr virus was discovered in Burkitt lymphoma tumor samples. The events leading to this discovery are captured by Burkitt* with characteristic modesty:

In 1961, I was lecturing to medical students at the Middlesex Hospital in London. The notice said something to the effect that an unknown guy from Africa would talk about an uninteresting tumor, if anyone happened to come along. For some reason Tony Epstein, who worked in a lab two blocks away, came and sat in the back. I had not spoken for more than 10 minutes when Tony recognized what I was saying as the missing piece of his jigsaw puzzle. He was looking for a human cancer that could be caused by viruses. He came up to me afterwards and asked if I could send him pieces of all our tumors. He would pay all the expenses, so I naturally accepted. We gave the tumors to the pilot in the BOAC plane, and he would bring them home to Tony, who would have them picked up at the airport.

For three years, Tony and his colleagues looked for viruses but could not find any. Then they got the idea that if, perhaps, they cultured the biopsy first, it might be more helpful. So they did culture the biopsy. The first slide Tony looked at after culturing was full of viruses. He was so excited he was afraid the electron microscope might burn up his slide, so he switched it off and walked around the block to cool his head. When he came back and switched it on again the viruses were still there. ${ }^{31}$

Epstein further tempered his enthusiasm in his initial 1964 report by stating that the virus probably represented a simple "passenger" in the tumor cells. ${ }^{81}$ However, the implications of this report were apparent.

In 1971, Manolov and Manolova discovered the characteristic reciprocal translocation between the long arms of chromosome 8 and

*From Burkitt DP: Discovering Burkitt's lymphoma: A special address to the Second International Symposium on the Epstein-Barr virus and associated diseases, in Levine PH, Ablashi DV, Nonoyama M, et al: Epstein-Barr Virus and Human Disease. Clifton, NJ, Humana Press, 1987. Used by permission. 
$14[t(8 ; 14)(q 24 ; q 132)]$ in Burkitt lymphoma cells. ${ }^{166}$ This central discovery was the first demonstration of what was later realized to be a mechanism for the activation of an oncogene, c-myc, in Burkitt's lymphoma ${ }^{64}$ Variant translocations between chromosome 8 and either chromosome 2 or 22, occurring in a minority of Burkitt lymphoma cases, would be found to have the same consequences. ${ }^{58-60}$ In each case, the c-myc oncogene on chromosome 8 would be brought into apposition with the immunoglobulin heavy (chromosome 14) or light (kappa 2, lambda 22) chain genes and be activated by mechanisms that are not yet clear. ${ }^{69,143,156}$ These different translocations apparently "freeze" the tumor cells at slightly different stages of maturation. ${ }^{79}$

By 1979, a model of a multistep mechanism for the development of Burkitt's lymphoma was proposed by Klein. ${ }^{144}$ According to this model, EBV infection of B-cells produced immortalized lymphocytes in vivo. Subsequently, chronic malaria resulted in B-cell stimulation secondary to the sustained presentation of antigen. Additionally, malaria induced a state of T-lymphocyte suppression that resulted in a decrease of T-cell control over B-cell proliferation. Given this background, continued cell proliferation favored the occurrence of a genetic accident. In this case, any of the several translocations involving c-myc could be associated with the entity known as Burkitt's lymphoma. Activation of additional oncogenes (e.g, B-lym $)^{72}$ was also considered likely in the further development of this disorder. ${ }^{143}$ The exact sequence of events and the role of the additional oncogenes are a matter of current debate. ${ }^{156}$

Lymphocytes infected with EBV do not normally form tumors when inoculated into the immunodeficient nude mouse ${ }^{180}$ Recently, it has been shown that insertion of the c-myc oncogene into lymphoblastoid cells infected with EBV resulted in tumorigenicity in this system, the degree of which was proportional to the amount of cmyc expression. ${ }^{163}$ The authors speculate that the interaction of these two factors, EBV transformation and c-myc activation, may provide a necessary and sufficient condition for malignant transformation of these cells. If this is so, then this model represents an in vitro recapitulation of the in vivo events leading to Burkitt's lymphoma, acquired immune deficiency syndrome (AIDS)-lymphoma and probably fully malignant PTLD as well. The model does not exclude the possibility of further or alternative forms of tumor progression. For example, evidence exists showing that EBV may activate other oncogenes indigenous to the host cell itself. ${ }^{206,254}$

Reminiscent of some cases of PTLD, there are documented instances of regression of Burkitt's lymphoma in humans. ${ }^{145}$ This phenomenon is also rarely observed in garden variety non-Hodgkin's lymphomas as well. ${ }^{151}$ 
Lymphoid Tumors in Congenital Immune Deficiency Syndromes

In 1969, Purtilo performed an autopsy on an 8-year-old male who succumbed to infectious mononucleosis. Over the following 6 years he diligently pursued the underlying pathogenesis leading to the unfortunate and similar deaths of five other males in this family. This work led to the elucidation of the X-linked lymphoproliferative syndrome (XLP) in $1975 .^{199}$ In this disorder, a specific defect exists in the host's ability to handle infection by the Epstein-Barr virus. ${ }^{204}$

Following infection with EBV, approximately two thirds of males with XLP will develop life-threatening or fatal infectious mononucleosis. ${ }^{201}$ In many of these cases, the cytotoxic T-cell response to $\mathrm{EBV}$-infected B-cells is uncontrolled, and this immunologic war leads to destruction of innocent bystander organs, notably the liver and bone marrow.

In approximately $35 \%$ of XLP cases malignant lymphoma may develop, due to uncontrolled B-lymphocyte proliferation following EBV infection. ${ }^{197}$ These tumors are very similar to PTLD occurring in allograft recipients, and they appear to have a similar pathogenesis.

Purtilo's hypothesis for the development of these lymphomas ${ }^{197}$ is based on an initial infection of B-lymphocytes by EBV. In the presence of immune hyporesponsiveness, an uncontrolled polyclonal tumor may develop. With continued proliferation a cytogenetic error occurs and leads to the overgrowth of a malignant monoclonal lymphoma. Ths schema has recently been expanded to accommodate the multistep theory of carcinogenesis. ${ }^{201}$ Thus, two alterations of the B-cell are postulated as the intermediate steps leading from a polyclonal to a monoclonal tumor. These "hits" on the B-cell may correlate to the oligoclonal tumors described as part of this syndrome ${ }^{201}$ and in transplant patients. ${ }^{45}$ The multiple hit concept also expands the hypothesis to accommodate the possibility that some proliferations, though of restricted clonality, may also be less than fully malignant.

The term "opportunistic cancer"201 has been applied to these and other malignancies arising in immunosuppressed patients. This term emphasizes the link to an infectious, i.e., viral, etiology and the necessity for host immune defects in such disorders. Thus, it is a particularly apt descriptor.

Using restriction-endonuclease probes, ${ }^{228}$ it has been possible to approximate the XLP locus on the sex $(\mathrm{X})$ chromosome. This opens the possibility of diagnosis of the disease prior to EBV infection and the use of immune prophylaxis (e.g., gamma globulin) in such cases.

Inherited immunodeficiencies in general represent a complex family of syndromes resulting from a large number of defects occurring during immune system development. Immunologically, they can be simply divided into defects of the T- or B-cell systems, or both. 
Lymphomagenesis in populations of congenitally immunodeficient patients has been referred to above. The overall risk of developing a malignancy with a congenital immune deficiency is currently estimated at $4 \%$, which represents a risk 10,000 times that of the normal population. ${ }^{127}$

In addition to reports from the Immunodeficiency Clinic in New York $^{62}$ (vide supra), the large Immunodeficiency Cancer Registry established at the University of Minnesota in $1973^{85,91,136}$ produced data that has recently been reviewed. ${ }^{127}$ We will not discuss this general subject further except to note that children with Wiskott-Aldrich syndrome, a clinically distinctive but pathogenetically obscure defect affecting both T- and B-cell systems, have the highest risk of developing malignancies, estimated at over $10 \% .{ }^{62,65,194}$ This is particularly poignant in light of the fact that these unfortunate children do not usually survive beyond the first years of life.

The most common tumors in all groups of congenitally immunodeficient patients are non-Hodgkin's lymphoma. Again, Purtilo ${ }^{197,200}$ has emphasized the etiologic role of EBV in a number of these tumors.

Similar to the case in transplant tumors, ${ }^{224}$ review of older reports of lymphomas in congenitally immunosuppressed patients has continuously modified and updated the pathologic interpretation. ${ }^{91}$ Thus, we agree with Bird and Britton ${ }^{15}$ that noncritical propagation of the earlier (circa-1960-1970s) literature on this subject may lead to fallacious conclusions. One case report of an EBV-associated lymphoproliferation in a congenitally immunodeficient patient serves to illustrate this. The case was originally considered to be polyclonal on the basis of immunophenotypic studies. ${ }^{212}$ Later studies of cells grown from this patient indicated a monoclonal component within the proliferation. The authors termed this an oligoclonal process, since other cells grown from the patient showed different clonal rearrangements. ${ }^{29}$ It is likely that many other cases would also be found to fit closely into the spectrum of lymphoproliferations described for XLP patients ${ }^{201}$ and transplant recipients ${ }^{90}$ if the original material were recoverable.

Some other cases have features suggestive, but not diagnostic, of an ineffective host response to EBV, sometimes with lymphoproliferative complications. Since T-lymphocytes constitute the major pool of proliferating cells in the host's response to EBV infection, the possibility also exists that these cells may also give rise to a malignancy. Aronson et al. ${ }^{7}$ have reported a T-cell leukemia, diagnosed by clonal analysis of the T-cell receptor, that occurred in a young male 1 year after an atypical EBV infection. They postulate that the leukemic cells, which demonstrated some features of NK cells, may have represented a neoplastic clonal outgrowth of reactive cells that had originally been mobilized to fight the infection. Sullivan et al. ${ }^{252}$ doc- 
umented an exuberant T-cell proliferation that could have been easily mistaken for lymphoma in a lymph node of a patient with virusassociated hemophagocytic syndrome. These authors considered this to be a manifestation of disordered host response to EBV infection in this case.

Very rarely, acute EBV infection may be associated with the appearance of non-Hodgkin's lymphoma in patients with no obvious immune deficiencies. ${ }^{221}$

\section{Lymphoid Tumors in the Acquired Immune Deficiency Syndrome}

An increase in B-cell lymphomas in AIDS patients was first recognized in $1982 .{ }^{209}$ By 1984, the clinicopathologic features of these tumors were delineated in a multi-institutional report of non-Hodgkin's lymphomas seen in 90 homosexual men with AIDS. ${ }^{272}$ The tumors arose as growths of monoclonal B-lymphocytes and were frequently, but not always, preceded by a lymphadenopathy syndrome (LAS) as part of the AIDS-related complex. ${ }^{157}$

The AIDS lymphomas differed from regular non-Hodgkin's lymphomas in that they tended to occur in extranodal locations (especially the central nervous system, bone marrow, and bowel) and responded poorly to therapy in many cases. ${ }^{239,272}$ In this regard, we ${ }^{244}$ noted the similarity between PTLD and AIDS lymphomas and suggested that the insights provided by the reversibility of transplant lymphoproliferations with reduction of immunosuppression might provide a stimulus to the use of immunoaugmentation techniques in AIDS-associated lymphomas. The fruits of this approach have not yet been realized, although the use of interferon to induce regression in AIDS-related Kaposi's sarcomas is becoming an accepted therapeutic modality. This response of Kaposi's sarcoma was also anticipated by the regressibility of similar tumors in transplant recipients subjected to a reduction of immunosuppression. $^{165}$

Subsequent studies of AIDS lymphomas have confirmed that their pathogenesis is strikingly similar to that of PTLDs. The polyclonal lymphadenopathy referred to above (LAS) was suggested as the soil from which monoclonal tumors could develop. ${ }^{271}$ Immunoglobulin gene rearrangement studies further revealed some of the LASs to be oligoclonal; that is, they were individual lesions composed of several proliferating B-cell clones. Rearrangement of the c-myc oncogene was observed in AIDS lymphomas but not in LAS biopsies. ${ }^{182}$ The HIV genome was not detected in lymphoma biopsies, suggesting that the virus did not play a direct part in the pathogenesis of this disorder. The current opinion is that these lymphomas may be a consequence of EBV infection in many cases. This is supported by evidence that the EB-viral load appears to increase in the saliva of AIDS patients as the severity of their underlying disease increases. ${ }^{2}$ How- 
ever, the role of EBV in the initial polyclonal activation of B-cells remains a matter of debate. ${ }^{57}$

The increase in AIDS-associated lymphoproliferations has led to closer study of the pattern of lymphoma development in general. One such study ${ }^{23}$ found an increase in the percentages of high-grade lymphomas and extranodal lymphomas in males with no known risk factors for AIDS. The study left open the question of whether or not this represented an absolute increase in these diseases, although the possibility was raised. This disturbing finding requires corroborative support. The role of EBV in these cases has not been investigated. An association of central nervous system lymphoma and EBV in patients with no evidence of immunodeficiency has also been reported. ${ }^{12}$

\section{Other Neoplasms in Immune Deficient States}

Other forms of cancer, not clearly associated with EBV, have also been reported in many of the populations at risk for EBV-induced neoplasia. Indeed, the preponderance of lymphoid tumors serves to distract attention from the milder increases in other forms of cancer. $^{18,118,124,125,136,138,189,241}$ It is not the purpose of this review to deal with cancers other than PTLDs, but one point relevant to transplantassociated neoplasia should be made in passing.

In the case of organ transplant recipients, there are extraordinary case reports of inadvertent transplantation of cancer cells that produced clinical disease in the recipient but regressed upon cessation of immunosuppression. ${ }^{95,266,274}$ One hepatic allograft recipient even rejected a donor hepatocellular carcinoma and its widespread pulmonary metastases. ${ }^{152}$ Unfortunately, this happy outcome is not the rule in most cases. ${ }^{231}$ However, it does demonstrate the power of the host immune response to reject foreign cells, even in some cases in which they are fully malignant.

The interested reader is referred to the reviews of Penn, ${ }^{185,188-193}$ Sheil ${ }^{229-231}$ and Birkeland ${ }^{18}$ for discussions of the general incidences of different types of neoplasia in transplant recipients. When comparing frequency rates of neoplasia from different transplant series, however, it is necessary to ascertain whether or not skin cancers are included in the statistics. These tumors, related to geographic locations in general and sun exposure in particular, may significantly increase the number of tumors reported from a particular center. ${ }^{103}$

\section{TRANSPLANT-ASSOCIATED LYMPHOPROLIFERATIONS}

\section{Introduction}

The existence of transplant-associated lymphoproliferations was discovered by Starzl in $1968 .{ }^{246}$ That same year, Penn ${ }^{192}$ instituted an informal registry of transplant tumors, located first in Denver and 
later in Cincinnati. In 1969, the first ${ }^{186}$ of a series of reports from this Center $^{185,187-193}$ defined the clinical dimensions of transplant-associated tumors. In 1970, the suggestion of reducing immunosuppression as a form of therapy for these tumors was first proposed. ${ }^{235,245}$

By the time of the description of X-linked lymphoproliferative disorder, it was also known that allograft recipients had increased oropharyngeal excretion of EBV. ${ }^{41,247}$ In 1975, Matas et al. ${ }^{170}$ hypothesized that the combination of Herpesvirus infection and iatrogenic immunosuppression was the cause of many of the tumors occurring in transplant recipients. In 1976, Matas et al. ${ }^{169}$ tentatively suggested an association between EBV and PTLD. A case report of diffuse lymphoma in a renal transplant recipient occurring in association with infection by EBV, Herpes simplex, and parainfluenza appeared in $1978 .{ }^{27}$ In that same year Iwatsuki, Geis, and their collaborators ${ }^{96,128}$ also raised the question of an association of EBV and PTLD. Their report is memorable in that the lesions in their five patients all resolved without the use of antitumor therapy.

In 1979, Marker et al. ${ }^{167}$ documented two cases of polyclonal PTLD arising in association with EBV infection. A similar association was soon reported by Nagington and Gray, ${ }^{177}$ who demonstrated EBV DNA within the tumor cells. In 1980, Crawford published a case report of an EBV-positive PTLD occurring in a Cyclosporin A treated renal recipient. ${ }^{56}$ Purtilo, in $1980,{ }^{203}$ suggested that immunosuppression of T-lymphocytes directed against EBV-infected B cells might provide a mechanism for the tumorous expansion of B-lymphocytes. These views were also adopted by Starzl et al. ${ }^{243}$

Ongoing collaborative efforts initiated by Purtilo and Klein and which centered on the University of Minnesota transplant population provided definitive evidence for the role of EBV in PTLDs. ${ }^{110,218}$ Similar results were later reported in studies of the transplant populations at Stanford and at the University of Pittsburgh. ${ }^{50,244}$

By 1981, Frizzera, extending his work on lymphoid tumors in congenitally immunodeficient patients, ${ }^{91}$ recognized that EBV-associated transplant lymphomas actually represented a spectrum of disorders ranging from benign to malignant. ${ }^{90}$ Attention turned to the clinicopathologic categorization of patient populations, ${ }^{107-111,244}$ earlier diagnosis, ${ }^{259,260}$ the specific role of EBV infection, ${ }^{121}$ and the clonal composition of the proliferations. ${ }^{45,50,178,244}$ Treatment modalities by this time included acyclovir, reduction of immunosuppression, surgical resection, chemotherapy, and radiotherapy in varying proportions. ${ }^{107-111,244}$

\section{Iatrogenic Immune Deficiency State of the Organ Transplant Recipient}

It would be pedantic to belabor the point that organ allograft recipients sustain a state of general immunosuppression in order to 
maintain a state of graft tolerance. Despite the fact that minor degrees of accommodation for coexistence occur on the parts of both the host and the allograft, ${ }^{135}$ it is the rare and unpredictable patient who develops a state of complete tolerance toward the transplant organ.

It is worthwhile, however, to point out that immunosuppressive regimens differ qualitatively as well as quantitatively. In light of the observation that differences in phenotypic expression of PTLDs exist among patient populations suppressed with different drug regimens, such differences may have clinical relevance.

One integrated model of immunologic sites subject to pharmacologic modulation is shown in Figure $1{ }^{135}$ It can be seen that separate but overlapping sites of action are proposed for the major immunosuppressive agents, cyclosporine (CsA), corticosteroids (S) and azathioprine (Aza).

Early reports of CsA use suggested that this agent was associated with a higher rate of lymphoma development than were other immunosuppressants. ${ }^{14,38,56}$ Further experience has not borne out these claims, ${ }^{15,37,255}$ even though the paralysis of T-cell function induced by this drug has been exploited in vitro to promote spontaneous outgrowth of EBV-infected B-lymphocytes. ${ }^{17,184}$ Cyclosporine inhibits mitogen- or alloantigen-induced lymphocyte proliferation, decreases release of IL-2 from T-cells, inhibits expansion of cytotoxic T-cell precursors, and inhibits the delayed hypersensitivity reaction. It also may selectively inhibit the T-dependent B-cell response and exhibit a relative sparing of $\mathrm{T}$-suppressor cell activities in certain instances. ${ }^{153,232}$

The purine analog azathioprine becomes active during metabolic degradation. The resulting thioinosinic acid inhibits DNA, RNA, and protein synthesis and acts particularly to inhibit the proliferative activities of T- and B-cells.

Corticosteroids bind to nuclear chromatin and can decrease the ability of lymphocytes to become activated, inhibit lymphocyte proliferation, and inhibit memory cell generation. An interaction between steroids and CsA has been shown. ${ }^{149}$ In addition, steroids exert other, unrelated effects on immune responses as well as on the expression of MHC antigens on the donor organ. ${ }^{113}$

Some biologic reagents are also used as immunosuppressants. The newest addition to our biologic armamentarium is the monoclonal antibody OKT $3{ }^{97}$ which binds to the CD3 antigen present on the surface of all T-cells. ${ }^{208}$ The CD3 (or T3) antigen normally acts to transduce the signal received by the cell when the T-cell receptor binds specifically to the antigen against which it is directed. The use of OKT3 antibody causes the CD3 molecule to disappear from the surface of the cell, resulting in cell paralysis. One major effect of this paralysis is the inhibition of T-cell cytotoxicity. This can be thera- 


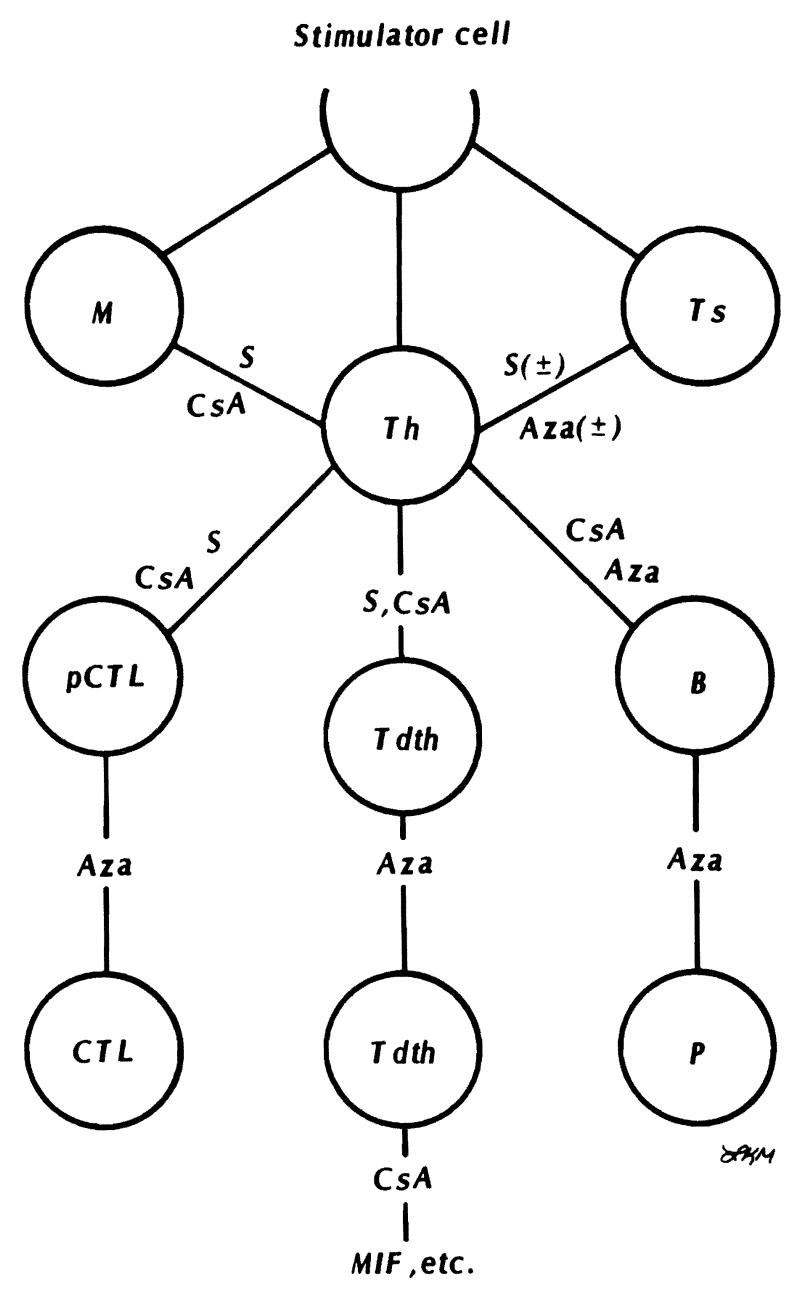

FIG 1.

Recognized sites of action of immunosuppressive agents in the allogeneic response. Aza, azathioprine; CsA, cyclosporin A; $S$, steroids; $M$, macrophage; TS, T suppressor lymphocyte; Th, T helper lymphocyte; $P C T L$, precytotoxic $T$ lymphocyte; $T d t h$, T delayedtype hypersensitivity cell; $B, B$ lymphocyte; $C T L$, cytotoxic T lymphocyte; $P$, plasma cell, MIF, migration inhibition factor; etc. includes other lymphokines associated with the delayed hypersensitivity response. (Adapted from Keown PA, Stiller CR: Control of rejection of transplanted organs, in Stollerman GH et al (eds): Advances in Internal Medicine, vol 31. Chicago, Year Book Medical Publishers, Inc., 1986. Reproduced with permission.). 
peutically useful for the reversal of acute rejection episodes. Our own experience with liver transplant recipients has substantiated this claim. ${ }^{92}$ One problem with this approach has been the development by the host of antibodies directed against the OKT3 antibody. ${ }^{97}$

Other biologic agents, such as Minnesota antilymphocyte globulin, are currently in use for the same purpose. The relative merits of OKT3 versus this agent have yet to be evaluated. All of these agents, even among themselves, show differential effects on circulating leukocytes. ${ }^{102}$

The list of drug effects could be extended further: cyclophosphamide shares many of the actions of azathioprine and the new and powerful experimental immunosuppressant FK506 (formerly FR900506 $)^{100 a}$ appears to function in a manner similar to, but more potent than, CsA. However, the significant observation is that each immunosuppressive agent has its own mechanism of action. In addition to site differences, differences probably also exist in regard to the reversibility of effect following withdrawal of drug. In this regard, the immunosuppressive effects of CsA have been shown to be rapidly reversible following withdrawal of the drug.

In attempts to compare PTLDs from different centers it is important to realize that the particular drug regimen used in a given series may be a confounding variable. Our own series is heavily skewed toward patients who have received CsA-containing regimens. ${ }^{244}$ The use of this drug at our institution has resulted in increased survival in general, due in large part to the better control of the acute rejection process within the first 6 months following transplant. ${ }^{99}$

\section{Risk Factors for the Development of Posttransplant Lymphoproliferative Disorder}

The occurrence of PTLD is dependent upon the presence of immunosuppression, Epstein-Barr virus infection, and other, poorly characterized factors.

The type of immunosuppression is not as important as its presence and intensity. In the Australia/New Zealand renal transplant series, a 3\% frequency of "lymphomas" was observed. ${ }^{229,230}$ These patients were treated with azathioprine, steroids, and occasionally antilymphocyte globulin. No figures are yet available for the frequency of PTLD with the use of OKT3, although the tumors have been documented to occur with the use of other monoclonal antibodies directed against the CD3 antigen. ${ }^{168}$ The extensive series of Penn ${ }^{185-193}$ also demonstrates the occurrence of tumors with all forms of antirejection immunosuppression.

In a separate study of 5,550 allograft recipients treated with CsA, the overall frequency of PTLD was $0.7 \% .^{12}$ This was identical to the $0.6 \%$ frequency within the subgroup of 4,000 renal recipients in- 
cluded in that series. However, when the renal subgroup was broken down by type of immunosuppression, differences were seen. Of 3,900 renal recipients treated with CsA and low dose steroids, a $0.4 \%$ incidence was seen, whereas this rose to $8.0 \%$ in 100 renal recipients treated with CsA combined with ALG, ATG, cytostatic drugs, or thoracic duct drainage.$^{12}$ On this basis Beveridge et al. stressed the need to avoid overimmunosuppression. They considered that maintaining blood trough levels of CsA between 200 and $1,000 \mathrm{ng} / \mathrm{ml}$, or serum trough levels between 50 and $200 \mathrm{ng} / \mathrm{ml}$, represented a desirable step in this direction.

A case report of PTLD occurring in a renal transplant patient receiving CsA-prednisone, despite meticulous attention to maintaining acceptable trough levels of CsA, was reported from the Yale series the following year. ${ }^{13}$ On the basis of immunologic function tests in this patient, the authors suggested that the actual degree of functional immunosuppression, rather than the blood level of drug, was the critical variable. Likewise, a separate case report documented two monoclonal paraproteins occurring in the presence of normal blood CsA levels. ${ }^{75}$ In this patient, who was receiving sulfinpyrazone, high doses of CsA were required to maintain normal serum levels of the drug. When the CsA dosage was lowered, the paraproteinemia abated.

The constitutional role of the host immune system in tumor development was analyzed in the Scandia Transplant material. ${ }^{19}$ Serial immune monitoring was performed in 10 patients who developed tumors following renal transplantation. Since only one of the tumors was lymphoid (Hodgkin's disease) and the immunosuppressive regimens did not include CsA, the results cannot be directly applied to CsA-associated PTLDs. Nevertheless, this pioneer study showed significantly decreased lymphocyte blastogenic responses in pretransplant specimens from patients who later developed tumors, when compared to a control group of transplant recipients who did not develop tumors. In a closely related area of investigation, Cunningham and Ascher $^{61}$ reported an increased prevalence of viral infections in patients in whom pretransplant CD4:CD8 (helper: suppressor) ratios were $\leq \mathbf{1 . 5}$ and who underwent conventional immunosuppression. The study stressed CMV infection and did not mention EBV. Similar results were not obtained when CsA-containing regimens were used.

Guttmann and Meakins ${ }^{105}$ followed renal transplant patients with serial skin testing. They found that 14 of 18 patients with tumors developing after transplant had been documented to be anergic for a mean period of 2.8 years before tumor diagnosis. However, most tumors in this series were nonlymphoid, and not every patient with anergy went on to develop tumor.

The role of EBV infection in the pathogenesis of PTLD was dis- 
cussed above. It should be emphasized that not every transplant patient with Epstein-Barr virus infection will go on to develop PTLD. ${ }^{30,117,121,154}$ Primary infection, ${ }^{121}$ infection in the face of high immunosuppression, ${ }^{30}$ and high virus shedder status ${ }^{268,269}$ appear to be likely candidates as variables whose presence augurs a significant risk of PTLD development.

Since EBV is strongly implicated in the development of PTLD, the question of case clustering arises. We have not examined this statistically in our own patient population, but have the impression that a weak temporal clustering may exist. More rigorously, however, Kinlen and Hoover ${ }^{137}$ were unable to find evidence of space-time clustering of PTLDs, despite a variety of statistical approaches.

An association between EBV infection and mean cyclosporine blood levels as risk factors for the development of lymphoma was elucidated in the Stanford heart and heart/lung transplant series. ${ }^{30}$ By EBV infection alone these investigators were unable to discriminate those patients who would go on to develop lymphoma from those who would not. ${ }^{117}$ However, when they coupled this with the mean CsA level, they were able to construct a logistic regression profile that to some degree separated out those patients at high risk for tumor.

Chronic antigenic stimulation from the graft itself has been suggested as a contributory factor in the development of PTLD. Small but measurable differences of tumor frequency are seen when recipients of different organ types are compared. Renal recipients generally have the lowest, and heart/lung recipients the highest, frequencies. At our institution, the present frequency of PTLD is $1.0 \%$ in renal transplant patients, $1.8 \%$ in heart, $2.2 \%$ in liver, and $4.6 \%$ in heart/lung recipients. Several qualifying remarks must be made, however. First, the allografts associated with the higher frequencies of PTLDs also require higher immunosuppression. This alone places the patient at increased risk. Second, one study of heart and heart/ lung recipients showed no difference in the number of rejection episodes between those patients who did and those who did not subsequently develop lymphoma. ${ }^{30}$ Third, a learning curve is probably operative in transplant centers, and early frequency estimates of PTLDs may later decrease as the denominator increases. For instance, the estimated $33 \%$ frequency in heart/lung recipients in our 1984 report $^{244}$ now stands at only $4.6 \%$ in 1987 . Last, in one series $^{229,230}$ there appeared to be a greater tendency for patients with cadaver kidney transplants to develop tumors than for patients with living related kidney transplants to develop tumors. This argued that the additional antigenicity of the unrelated cadaver kidney may have played a pathogenetic role in this process. However, subsequent correction for age showed that the two patient groups were equally susceptible to cancer. ${ }^{231}$ In contrast to these findings, Birkeland has 
reported a relationship between tumor development and degree of HLA mismatch. ${ }^{18}$ However, this Scandinavian series included a large number of nonlymphoid tumors.

Regardless of these disclaimers, our own cases indicate that the lesions may initially present in the allograft organ, suggesting a cause and effect relationship. A tantalizing case report of a lymphoproliferative disorder occurring in a bone marrow recipient documented the emergence of a neoplasm (of host origin) shortly after the appearance of a Coombs' positive hemolytic anemia. ${ }^{21}$ One obvious inference is that the blood group mismatch stimulated host cells to produce antibody to the donor blood group antigen. From this stimulated B-cell pool a malignant clone emerged. Curiously, EBV could not be documented in this tumor despite the presence of reactivation infection. The absence of virus, which has occasionally been noted ${ }^{121}$ may represent a technical artifact or may point to other, unknown cofactors in the development of PTLD. We ${ }^{68}$ have shown atypical lymphoid hyperplasia in nontransplanted rats who were fed oral CsA. This finding had not previously been reported ${ }^{84,256,257}$ These results are of interest since EBV does not have the ability to infect this species.

In the case of bone marrow transplants, the risk for PTLD is significantly increased when T-cell-depleted mismatched donor marrow is used. ${ }^{226}$ In one series, ${ }^{226}$ a $24 \%$ frequency of PTLD was seen in this setting, compared to approximately $0 \%$ frequency when either matched T-cell-depleted or matched non-T-cell-depleted marrow was used.

The association of the original underlying disease that led to transplant and the propensity to develop PTLD has not been analyzed in our series. Some investigators have put forth statistical data to support such an association for the conditions of idiopathic cardiomyopathy $^{3,14}$ or polycystic kidneys ${ }^{124}$ and subsequent tendency to develop PTLD. An association between chronic interstitial nephritis and tumor development (including non-PTLD types) has also been suggested. ${ }^{18}$

\section{Pathology of Posttransplant Lymphoproliferative Disorder}

Macroscopic Appearance.-PTLDs demonstrate a number of appearances depending upon their growth patterns and locations. In CsA-associated proliferations, most cases present with mass lesions, but a minority of PTLDs may assume an infiltrative form of growth. Tumorous lesions often contain a prominent necrotic component, a feature that may also be of use in the radiologic evaluation of these patients. ${ }^{263}$

In our series, the head and neck and the gastrointestinal tract were the two areas most commonly involved by PTLDs. In the head 
and neck, tonsils, lymph nodes, and salivary glands were the usual sites of tumor. The brain appears to be a target organ for the disease only in those allograft recipients who have received conventional (non-CsA-containing) immunosuppression. ${ }^{185,193}$ In the Minnesota series, $14 \%$ of patients had disease confined to the CNS and a total of $45 \%$ had CNS disease, usually in association with widespread disease. $^{108}$

The tonsils, when involved, usually enlarge quite dramatically, often contain prominent necrosis and may cause acute respiratory embarrassment. Involved lymph nodes in the early stages maintain a smooth, circumscribed capsule and on cut surface display a uniform, gray-white to tan surface. The presence of necrosis is variable. With more advanced lesions, the capsule is breached, and the proliferation invades into the surrounding soft tissues with attendant difficulties in any surgical resection. The appearance of the fully involved node is indistinguishable from that of diffuse lymphoma.

In the neck, tumors may also arise from the salivary glands. It is not certain whether these arise from salivary gland de novo or from lymph nodes in an intrasalivary location.

As a general rule, the histologic changes in individual cases appear to be more fully expressed in the largest lymph nodes. This fact should be remembered when selecting a node for biopsy. Also, patients with predominant head and neck disease may have generalized adenopathy in addition to their major disease focus. The histologic changes in lymph node biopsies taken from areas far removed from the main area of disease may be easily dismissed by the uninitiated as nonspecific reactive hyperplasia. This serves as another caveat to the surgeon in selecting a biopsy site (see histologic changes, below).

Tumors involving the gut tend to be large, rapidly growing, and multiple (Fig 2). These lesions are seen more frequently in patients receiving CsA-containing regimens, ${ }^{185,244}$ although they are seen occasionally with conventional immunosuppressive regimens. ${ }^{129,134}$ Eleven of our first $\mathbf{4 4}$ patients had a primary gastrointestinal manifestation of PTLD. Involvement of areas rich in gut-associated lymphoid tissue is the usual case, although individual tumors have been seen in the stomach. The propensity of the tumors for local invasion and consequent tissue necrosis can quickly lead to perforation of the bowel wall; multiple perforations are not uncommon in untreated cases. Perforations may cause a free rupture into the peritoneal cavity or may result in a lárge mass composed of tumor and matted loops of bowel with signs and symptoms of complete or incomplete intestinal obstruction.

Viewed from the mucosal aspect, the fully developed intestinal PTLD presents an ulceronodular mass with a green-yellow pseudomembrane. In small lesions, the epicenter appears to arise from the 


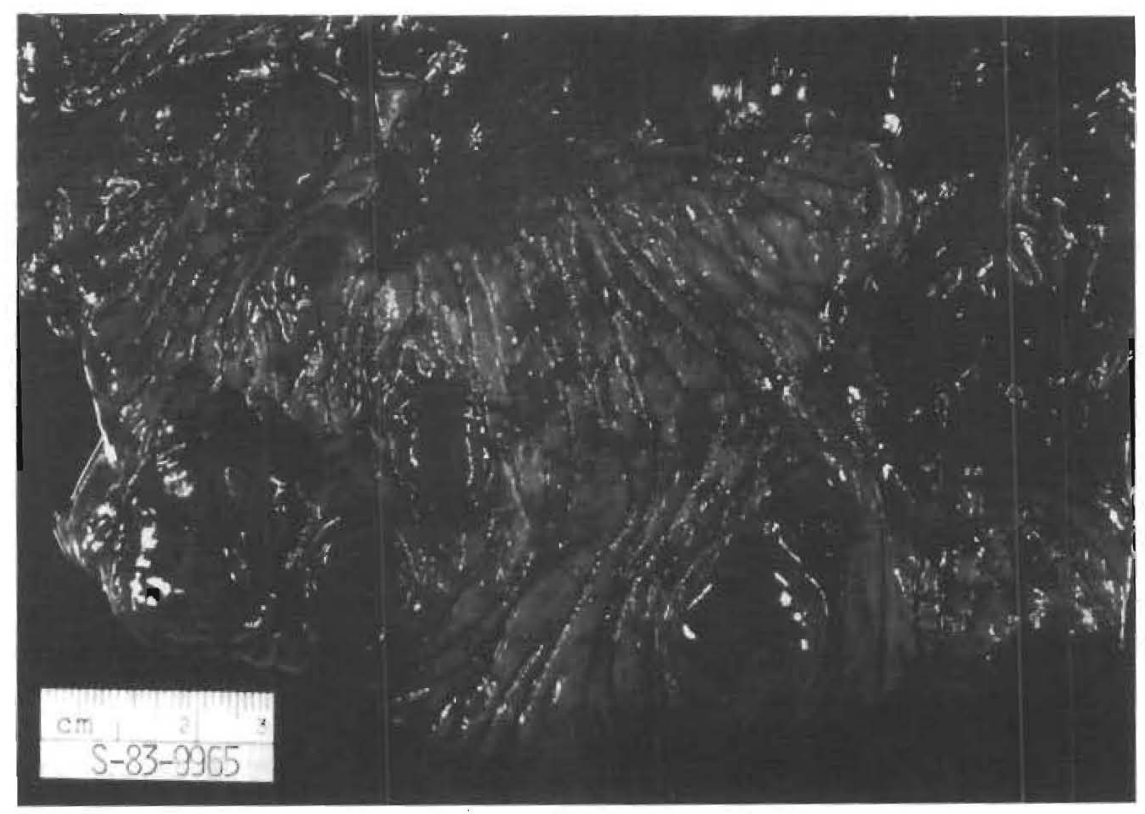

FIG 2.

Region of ileocecal valve and ascending colon from a liver transplant recipient with PTLD The specimen has been opened and is viewed from the mucosal aspect. Multiple ulceronodular tumors are evident. The patient is alive and well 4 years following tumor resection and reduction of immunosuppression. (From Makowka L, Nalesnik MA, Stieber A, et al: Control of post-transplant lymphoproliferative disorders and Kaposi's sarcoma by modulation of immunosuppression, in Good RA, Lindenlaub E (eds): Symposia Medica Hoechst: The Nature, Cellular, and Biochemical Basis and Management of Immunodeficiencies. Stuttgart-New York, FK Schattauer Verlag, 1987, pp 567-624. Reproduced with permission.)

submucosa. In larger Jesions the entire thickness of the bowel wall is obliterated by tumor. In one case in which clinical regression was documented (case 4, below), resection of regressing tumors showed shallow ulcers with white membranes forming on the surface of the ulcer. These membranes represented regenerating mucosa.

In the lungs the tumors have a nodular shape and may be either single or multiple. This appearance is also appreciated on X-ray examination (see case 5, below). On cut surface the lesions are graywhite, again raising the pathologic likelihood of a lymphoma. These lesions very often occur in association with nearby pneumonia. In our series, Pneumocystis carinii or CMV represented the organisms most often involved in this process. Pulmonary lesions may be the only site of disease or may be seen in association with disease in other organs.

Lesions in solid organs may be tumorous or infiltrative. Tumors 
are lobulated, gray-white and often contain obvious areas of necrosis. Infiltrative lesions may be inapparent on gross examination, or may impart a mottled appearance to the organ. The variegated appearance may be further accentuated by necrosis of the parenchymal tissue. If the organ is an allograft, the possibility of rejection may be raised on gross examination. This is also a differential diagnostic consideration in the microscopic analysis.

Microscopic Appearance.-It is well known that the lymphoid lesions associated with EBV infection may mimic malignant lymphoma of either Hodgkin's ${ }^{172,219}$ or non-Hodgkin's type. ${ }^{42,74,78,101,219}$ This feature appears to be shared among several of the Herpes viruses, since Herpes lymphadenitis may, on occasion, also mimic lymphoma. ${ }^{9}$

The term posttransplant lymphoproliferative disorder is used to refer to a spectrum of entities that merge into the histologic pattern of infectious mononucleosis on the one hand and the histologic pattern of true non-Hodgkin's malignant lymphoma on the other. The relation of Hodgkin's disease to PTLD is uncertain at this time. Hodgkin's disease has uncommonly been reported in transplant recipients ${ }^{76,187,188}$ and is not increased in frequency in congenitally immunodeficient states. ${ }^{85}$ Likewise, the relationship of PTLD to the rarely occurring T-cell lymphomas in transplant patients is also unclear. $^{132,162}$

In essence, PTLD is an uncontrolled or poorly controlled proliferation of lymphocytes. The cells grow diffusely, often causing destruction of underlying structures. This appearance is subject to considerable modification dependent upon the balance between the state of tumor progression and host response. A full histologic report of PTLD should include evaluation of: (1) the nature of the growth pattern (diffuse, invasive, etc.); (2) the degree of necrosis; (3) the polymorphic/monomorphic nature of the cells; (4) the presence or absence of plasmacytoid differentiation; and (5) the presence or absence of atypical mononuclear blast forms. The nodal or extranodal location of the tumor should be specified. The presence or absence of retention of underlying architecture should also be commented upon.

A diffuse pattern of growth is characteristic of PTLD. ${ }^{90}$ Uncomplicated infectious mononucleosis involving lymph nodes usually shows a combination of diffuse areas and residual nodular areas, which represent germinal centers, although the latter may be apparent only by the use of special stains. ${ }^{42}$ Much attention has been given to the differentiation of PTLD from garden variety malignant lymphoma. It may be equally difficult to distinguish PTLD from infectious mononucleosis. The difference appears to be quantitative rather than qualitative and in the iatrogenically immunosuppressed 
patient may be a matter more of academic than of clinical relevance.

Necrosis is often a prominent finding of PTLD ${ }^{90}$ (Fig 3). Large areas of confluent necrosis may reflect the gross impression. In other cases individual cell necrosis predominates. The cause of necrosis is probably related to a combination of rapid cell growth, destruction of nutrient blood vessels by angioinvasive lymphoid cells, host immune effector mechanisms, or lytic infection of some of the cells. Similar degrees of necrosis have been observed in some cases of XLP. ${ }^{198}$ The relative contributions of each of these factors and the possible existence of other mediators of tumor necrosis have not yet been evaluated.

The main cellular constituent of the PTLD is the B-lymphocyte. This cell normally varies in size and shape as it undergoes differentiation. B-lymphocytes at all stages of differentiation may be seen within a PTLD. The term polymorphic has been applied to characterize the mixed appearance that may result from this phenomenon. ${ }^{90}$ A polymorphic appearance is thus composed of small lymphocytes, small cleaved, large cleaved, small noncleaved, large

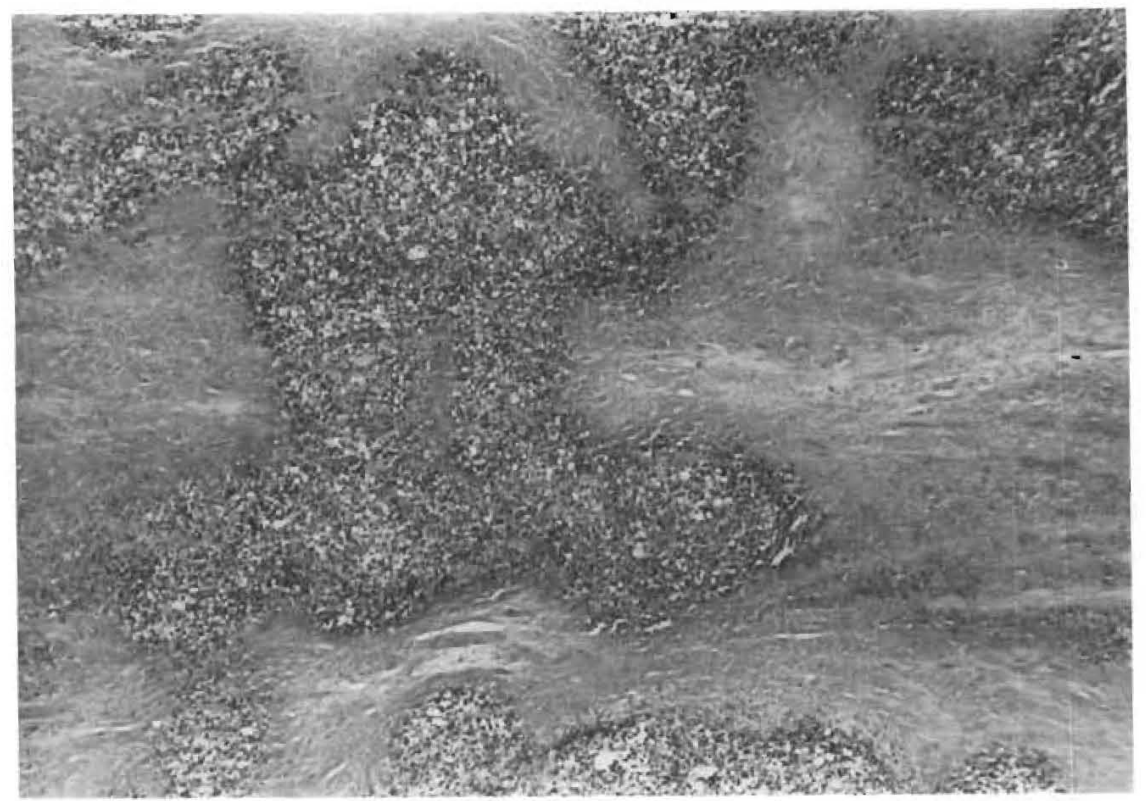

\section{FIG 3.}

Low power photomicrograph of PTLD demonstrates large areas of confluent necrosis ( $\mathrm{He}$ matoxylin-eosin stain). (From Makowka L, Nalesnik MA, Stieber A, et al: Control of posttransplant lymphoproliferative disorders and Kaposi's sarcoma by modulation of immunosuppression, in Good RA, Lindenlaub E (eds): Symposia Medica Hoechst: The Nature, Cellular, and Biochemical Basis and Management of Immunodeficiencies. Stuttgart-New York, FK Schattauer Verlag, 1987, pp 567-627. Reproduced with permission.) 
noncleaved lymphocytes, immunoblasts, and plasma cells (Fig 4). Infectious mononucleosis, as may be expected, also produces a variety of B-lymphocyte forms. Few other entities demonstrate a diffuse proliferation of such a wide range of B-lymphocytes.

In addition to the polymorphic display of B-lymphocytes, large bizarre cells may be seen. These cells have been called atypical immunoblasts (Fig 5). ${ }^{90}$ They may resemble Reed-Sternberg cells, and similar cells have also been described in uncomplicated infectious mononucleosis. ${ }^{42,74,101,164,172,219,249}$ In CsA-associated proliferations, such cells usually constitute a background finding in PTLD. They are often, but not always, seen in association with areas of necrosis, suggesting that the appearance may represent a response of the cell to a toxic microenvironment. In patients who develop PTLD in association with routine immunosuppression, such cells may occur in more abundant numbers and have been interpreted as malignant cells. Our own studies indicate that the cells have no prognostic significance in CsA-associated PTLDs. Further studies are required to clarify the range of cells that constitute this histologic entity.

The most important histologic feature, at least in CsA-associated PTLDs, is the distinction between monomorphic and polymorphic proliferation. The polymorphic mixture of lymphocytes at varying

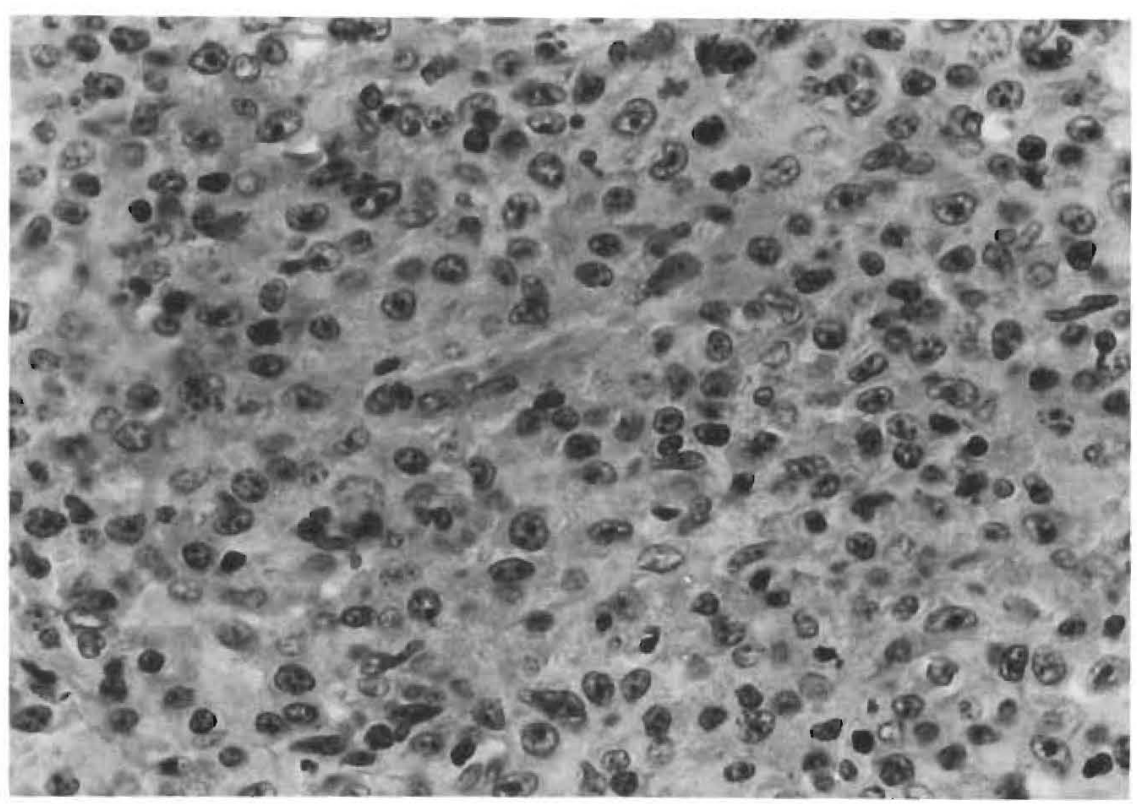

FIG 4.

Representative polymorphic PTLD. In this high-power photomicrograph, the diffuse proliferation of lymphoid cells shows considerable variation in size and shape. (Hematoxylineosin stain). 


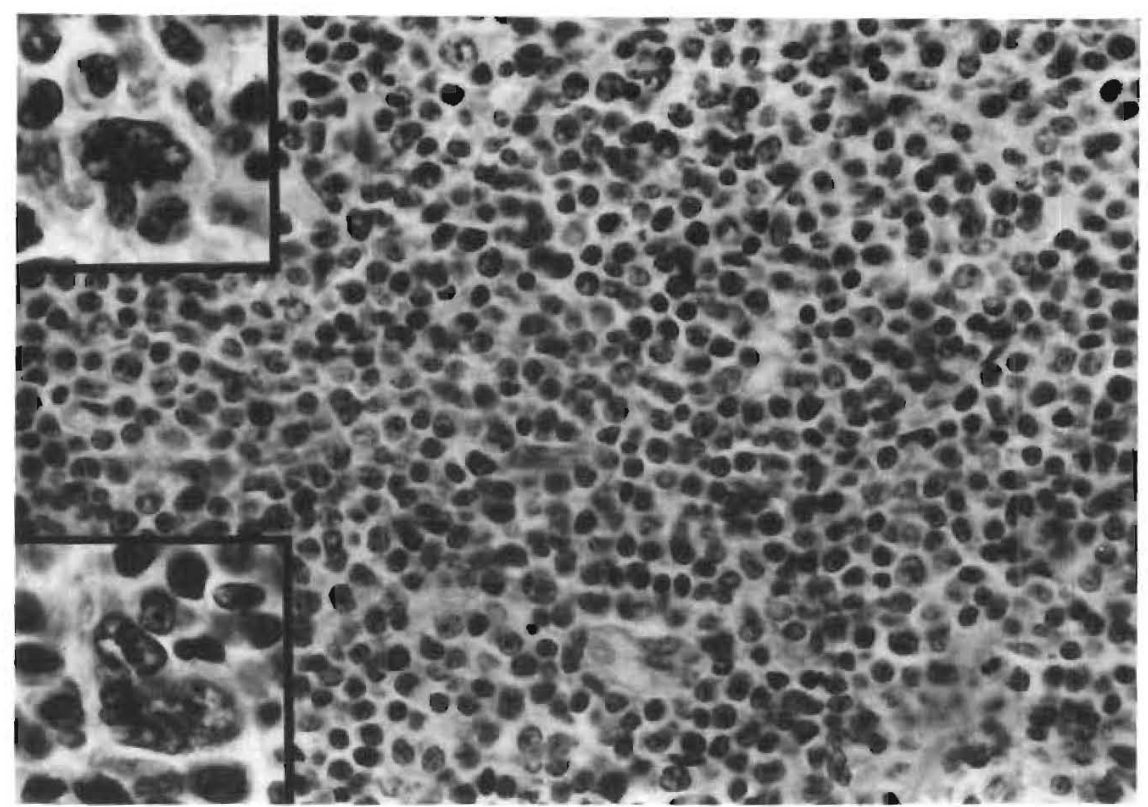

FIG 5.

Minimally polymorphic PTLD which was shown to be monoclonal by immunoglobulin gene rearrangement analysis. Inset: rare large and atypical-appearing lymphoid cells found within the tumor. (Hematoxylin-eosin stain. From Makowka L, Nalesnik MA, Stieber A, et al: Control of post-transplant lymphoproliferative disorders and Kaposi's sarscoma by modulation of immunosuppression, in Good RA, Lindenlaub E (eds): Symposia Medica Hoechst: The Nature, Cellular, and Biochemical Basis and Management of Immunodeficiencies. Stuttgart-New York, FK Schattauer Verlag, 1987, pp 567-624. Reproduced with permission.)

levels of differentiation was one feature that first led Frizzera to realize that the lesions were not identical to non-Hodgkin's lymphoma. ${ }^{90,110} \mathrm{~A}$ polymorphic proliferation is also used as one distinguishing feature between uncomplicated infectious mononucleosis and non-Hodgkin's lymphoma ${ }^{42}$ Conversely, the earliest report of PTLD from the Minnesota series ${ }^{169}$ stressed the monomorphic appearance of two cases.

In our cases, the majority of lesions display a polymorphic pattern. In a minority of tumors, however, all cells appear to be at the same level of maturation. This monomorphic appearance suggests a divergent stage in tumor development (Fig 6). The uniformity of cells is strong circumstantial evidence that a single clone has emerged from a polyclonal background due to an expression of those characteristics conferring a selective growth advantage. The actual situation is slightly more complex and is considered below in the discussion of the relationship of tumor clonality and histology. 


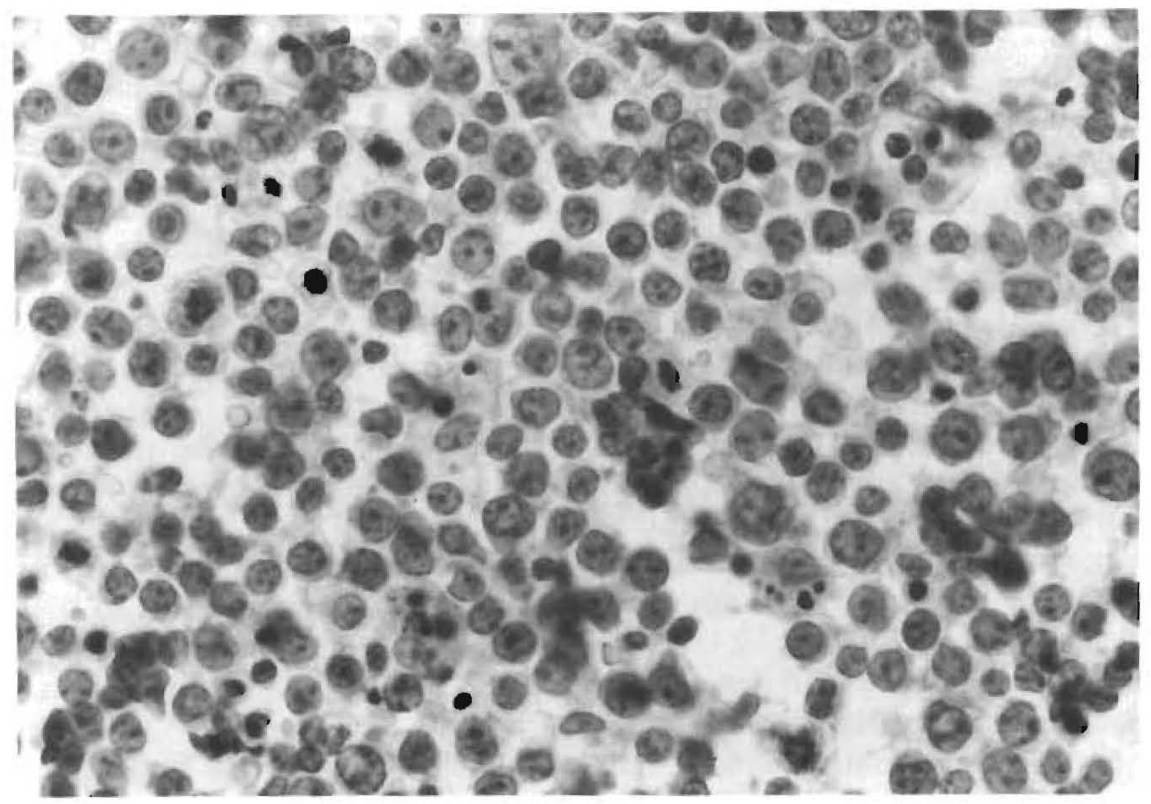

FIG 6.

Touch imprint preparation from a monomorphic PTLD. In this case, there is significant homogeneity in the appearance of the nuclei. Mitotic activity is apparent. Scattered macrophages containing ingested material can also be seen (hematoxylin-eosin stain). This cervical tumor was monoclonal by immunophenotypic and immunogenotypic analysis. The patient is alive and well following local resection and reduction of immunosuppression.

An appearance between that of the polymorphic and monomorphic conditions has been seen by us on several occasions. This appearance is seen in lesions containing a predominance of plasma cells and plasmacytoid cells. It may be seen in at least two separate settings. In the first case, a mature, diffuse plasma cell infiltrate may be seen in lymph nodes in association with more typical PTLD elsewhere. It is not clear whether this benign-appearing infiltrate represents a reaction to infection elsewhere or the earliest manifestation of PTLD. In any case, this appearance should prompt a thorough evaluation for EBV infection and the presence of occult mass lesions.

In the second instance, this appearance has been seen in association with regression of lesions. It may be that regression in such cases is associated with maturation of a population of B lymphocytes. This plasmacytic appearance may be infiltrative and is accompanied by the residua of previous damage due to the lesion. The cells may be interpreted as signifying an inflammation at this point if read out of context. (The process of B-cell maturation in PTLD may represent a benign counterpart of multiple myeloma. In this latter disease, evidence has been presented to suggest the maturation of 
monoclonal lymphocytes into plasma cells. ${ }^{173}$ In multiple myeloma, of course, the maturation is not associated with regression).

Secondary microscopic changes may vary according to the location of the tumor. In the gut, the overlying ulcer often shows intense overgrowth of bacteria. It is possible that antigenic stimulation by these microorganisms may confer a selective proliferative advantage upon cells with the appropriate antibody specificity. No studies have yet addressed this possibility.

In solid organs, parenchymal necrosis may be seen in conjunction with tumor. In one of our cases, a moderate portal infiltrate was associated with almost complete hepatocellular necrosis.

Our interpretation of CsA-associated PTLDs stresses the distinction between polymorphism and monomorphism as being of primary importance. The first, and most widely used, classification system, however, is that of Frizzera, ${ }^{90}$ who recognized a continuum of histologic disease in this syndrome. The spectrum delineated in his classification consists of nonspecific reactive hyperplasia, polymorphic diffuse B-cell hyperplasia, polymorphic B-cell lymphoma, and immunoblastic sarcoma.

In this classification system, polymorphic diffuse B-cell hyperplasia (PDBH) represents a diffuse and invasive proliferation of B-lymphocytes at varying stages of development. Progression to polymorphic B-cell lymphoma (PBL) is heralded by the appearance of confluent (geographic) necrosis and atypical immunoblasts. Immunoblastic sarcoma is described as a terminal histologic stage of malignancy.

In our opinion, we are describing the same histologic spectrum originally seen by Frizzera. We recognize both PDBH and PBL as progressive steps in this development, but lump them together as polymorphous PTLD, since in our patients both forms are usually easily controllable by the same therapeutic approach. The immunoblastic sarcoma of Frizzera most likely corresponds to what we would call monomorphous PTLD. Since monomorphous PTLD may resemble non-Hodgkin's lymphoma other than immunoblastic sarcoma, we prefer not to use this latter term indiscriminately.

Clonal Composition of Posttransplant Lymphoproliferative Disorder

The term PTLD as used refers to a range of nonclonal to clonal proliferations. The nonclonal proliferations are also referred to as polyclonal and the clonal tumors referred to as monoclonal. For most cell types, clonal lesions represent malignancies and nonclonal proliferations represent hyperplasias. With increased ability to detect clonal components, these distinctions are becoming blurred in areas. However, they still remain useful generalizations.

The earliest description of transplant lymphomas considered them to be non-Hodgkin's lymphomas and, by inference, clonal. 
Geis, in 1978, first applied the term multiclonal to these proliferations. ${ }^{96}$ In 1979, a major collaboration among the Universities of $\mathrm{Ne}$ braska and Minnesota and the Karolinska Institute was initiated for the purpose of more fully analyzing these lesions. ${ }^{200}$ Out of this work came the discovery, based on immunocytochemical studies, that both clonal and nonclonal PTLDs existed. These workers integrated their findings by hypothesizing that an initial polyclonal proliferation provided the substrate from which a malignant monoclonal proliferation could emerge. A case report was later offered to demonstrate the transition from (benign) polyclonal to (malignant) monoclonal PTLD. ${ }^{109 a}$

A second set of studies was performed at Stanford University in 1983 utilizing immunoglobulin gene rearrangement techniques of both heavy and light chain genes. In this study of tumors arising in 10 heart recipients, evidence of clonal rearrangements was found in each case ${ }^{50}$ The utility of this technique was underscored by the fact that prior immunophenotypic analysis failed to make any determination of clonal status in nine of the cases. On the basis of these findings the investigators considered the tumors to be neoplastic from the outset. This conclusion regarding clonality was buttressed by the discovery of clonal gene rearrangements in eight specimens sent to Stanford from the University of Pittsburgh series in November 1983. The Stanford researchers were unable to reconcile their findings with the findings of others ${ }^{90,244}$ that early lesions may be nonclonal at presentation. Further analysis of some of their patients who had multiple concurrent tumors indicated that each separate tumor constituted a unique monoclonal proliferation. ${ }^{45}$ In this study of five patients, individual cases had between two and six separate clonal proliferations. Only rarely did tumors at different sites demonstrate identical patterns. The authors favored the hypothesis that the multiple clones represented derivatives from a progenitor tumor, rather than separate independent primary tumors. The detection of differing clonal rearrangements led these investigators to apply the term "multiclonal" to the family of proliferations. Later work from the Stanford group focused upon clarification of this phenomenon in the more readily available non-Hodgkin's B cell lymphomas from nonimmunosuppressed patients. They first observed that a significant minority of such cases also demonstrated several clones. ${ }^{159,234}$ This appeared to challenge the concept that lymphomas are necessarily monoclonal. A later study showed that an important mechanism for this diversity, at least in some cases, is the ability of the Bcell to undergo continuing mutations at certain regions of the immunoglobulin genes, even after having reached a neoplastic state. ${ }^{48}$ These findings suggest, first, that the multiple tumors seen in such cases are probably derived from a progenitor clone and, second, that an active process of clonal selection (i.e., "survival of the fittest") may 
be ongoing in established tumors. We have also detected multiple related clones from one case of multiple synchronous PTLDs in our series, suggesting that a similar mechanism may be operative in this disorder as well (Locker, unpublished observations, 1987). Multiple clonal as well as nonclonal EBV-associated tumors have also been described in a case of severe combined immunodeficiency syndrome (the "bubble boy" case) ${ }^{227}$ Most recently, the Stanford group has acknowledged the probable existence of polyclonal PTLDs, but continues to stress that occult monoclonal components may be detectable only by DNA gene rearrangement methodology. ${ }^{47}$

The first major summary of the University of Pittsburgh PTLD experience appeared in the Spring of $1984 .^{244}$ We documented the clonality of PTLDs in the first 17 patients in our series, based on immunocytochemical techniques. The immunoperoxidase studies were concurrently performed in Pittsburgh and at St. Mary's Medical School in London by Professor K. Porter. One of the authors (MN) vividly remembers how a long comparison of results tabulating the frequency of positively staining cells agreed number for number, almost perfectly, despite the fact that the procedures were performed almost 3,000 miles apart, using different reagents, personnel, and laboratories. On the basis of these studies we concluded that both polyclonal and monoclonal PTLDs did indeed exist.

We were also the first to report a somewhat unexpected, but seminal, finding that a reduction of immunosuppression was capable of inducing regression in some monoclonal, as well as polyclonal, tumor. ${ }^{244}$ Because of the pivotal nature of this observation in regard to the role of immune control mechanisms and even the ultimate definition of malignancy, the results were greeted with some degree of skepticism. ${ }^{78}$ This doubt was in large part due to the uncontrolled nature of the clinical series and to the fact that clonal determinations were based on immunocytochemical techniques and not the newly described and more sensitive gene rearrangement procedures.

We have since applied immunoglobulin gene rearrangement methodology to a number of cases in our series. The results have confirmed our initial observations. On the basis of a combined immunogenotypic and immunophenotypic analysis of lesions in our first 43 patients with PTLD, 28\% had monoclonal tumors, $30 \%$ had polyclonal tumors, and $12 \%$ had separate clonal and nonclonal lesions. Clonal determinations were inconclusive in the remainder due to insufficient tissue samples ${ }^{178}$ (Nalesnik et al., submitted).

In one case in our series, clonal analysis of multiple tumors by evaluation of immunoglobulin gene rearrangement was complemented by clonal analysis of the EBV genome ${ }^{205}$ (Locker et al., manuscript in preparation). Preliminary results suggest a primary clonal 
proliferation of a cell that had not yet arranged its immunoglobulin genes. (Infection of pre-B cells by EBV has previously been documented to occur in uncomplicated infectious mononucleosis, ${ }^{28}$ but the earliest known B-precursor has already rearranged its immunoglobulin genes). ${ }^{112}$ As the cell continued to mature, immunoglobulin gene rearrangement occurred and derivative clones developed as separate tumors. If this example holds up, it indicates that some lesions referred to as nonclonal, even by immunoglobulin gene rearrangement studies, may be clonal proliferations if the lymphocyte involved has not yet rearranged its immunoglobulin genes. However, in other cases in our series, separate clonal and nonclonal proliferations were observed within the same patients. A similar finding of clonal and nonclonal proliferations has been reported in at least one case from the Minnesota transplant series. ${ }^{107}$

One patient in our series appeared to have had a clonal population existing within a polyclonal background. A similar situation appears to have been described in a patient with Wiskott-Aldrich syndrome. ${ }^{6}$ The authors of this report, in discussing their case, have also questioned whether the monoclonal component was necessarily equated with malignancy. ${ }^{6}$ A separate study of another patient with this syndrome has documented the presence of two separate monoclonal B-cell tumors, ${ }^{65}$ further indicating the similar pathogenesis of lymphoproliferations arising in either congenital or acquired immune deficiency states.

No difference in the transplant to tumor time interval was seen in our patient series when clonal and nonclonal lesions were compared. This indicates that either some tumors begin as monoclonal and some as polyclonal lesions, or that a progression from polyclonal to monoclonal tumors occurs very rapidly in these patients. A progression of monoclonal to polyclonal tumor runs counter to current concepts of tumor progression and is extremely unlikely. Indeed, we agree with Harris ${ }^{39}$ that the term polyclonal lymphoma ${ }^{78}$ should be avoided as a designation for this pathologic entity.

In contrast to our findings, Hanto et al. ${ }^{108,109}$ have observed a more clear-cut temporal segregation between patients who developed either polyclonal or monoclonal disease under the influence of conventional immunosuppression. In their series, patients with polyclonal disease presented, on the average, within 1 year after transplant and those with monoclonal disease presented at a mean time of 5.3 years after transplant. ${ }^{107}$

Karyotypic Analysis of Posttransplant Lymphoproliferative Disorder

Given the similarities in the proposed pathogenetic mechanisms operative in both Burkitt's lymphoma and PTLD, one might hypothesize that most if not all of the monoclonal PTLDs would exhibit the 
8;14 translocation (or one of its variants) typical of Burkitt's lymphoma. Unfortunately, from a reductionist standpoint, this is not the case. Indeed, as far as we are aware, there is only one case of monoclonal PTLD from our series that has demonstrated a reciprocal $\mathbf{t}(8 ; 14)$ (Jaffe, Nakazato, manuscript in preparation).

Intriguing karyotypic data on 6 of 22 patients have been presented $^{107,108}$ from the Minnesota series. One of these patients with a monoclonal tumor of the cervix showed a translocation between the short arms of chromosome 2 and 19 in all cells examined. Four months later, a metastatic tumor from this patient showed this same translocation with an additional second translocation between the long arms of chromosome 6 and 16. This suggests tumor progression over this time period with the development of a more malignant cancer.

A second patient in this series presented with a polyclonal PTLD but demonstrated trisomy 3 in four of 14 cells examined. This was interpreted as representing the emergence of a monoclonal proliferation of cytogenetically abnormal cells within an otherwise polyclonal tumor. Despite this worrisome feature, the patient survived with no evidence of disease following a course of acyclovir and a discontinuation of immunosuppressive therapy. Interestingly, this was the only patient of the series who was maintained on a CsAcontaining immunosuppressive regimen.

A third patient, with a trisomy 14 in approximately $50 \%$ of cells in a polyclonal PTLD, did not fare so well. Despite a discontinuation of immunosuppression and the institution of antilymphoma therapy, this patient expired 15 months later with metastatic disease and sepsis. Initial immunosuppression included azathioprine, prednisone, antilymphocyte globulin, and local graft irradiation. ${ }^{83}$

A fourth patient of particular interest had separate monoclonal and polyclonal PTLDs. ${ }^{107}$ Both tumors displayed a normal karyotype. This prompted the authors to suggest that (a) polyclonal-to-monoclonal conversion actually preceded the appearance of cytogenetic abnormality or (b) the cytogenetic abnormality was not necessary for malignant transformation in this case. ${ }^{108}$ We would consider that both of these possibilities may be operative in a given case. Based on our experience, monoclonal PTLDs may be seen in the absence of cytogenetic abnormalities. A monoclonal proliferation of this sort, however, might better be referred to as an "opportunistic cancer" or "conditional neoplasia" to emphasize the necessity for the presence of an immunocompromised host. Malignant transformation might better be used to refer to the fully developed malignant phenotype that is a necessary precondition for the onset of a true cancer in an immunocompetent individual.

Tumor cell karyotype has also been used on occasion to docu- 
ment the host or donor origin of the PTLD. Most reported cases have shown tumor cells to arise from the host population. ${ }^{188,189}$ However, in bone marrow transplant recipients, tumors may arise from either host $^{21}$ or donor ${ }^{100,168,222}$ cells.

Recently, it has been found that many non-Hodgkin's B-cell lymphomas contain specific chromosome translocations. ${ }^{270}$ Translocations identical to these have not been described at this time in PTLDs.

\section{Oncogene Expression in Posttransplant Lymphoproliferative Disorder}

Studies of oncogenes or their products have not yet been reported from the major series of transplant-associated lymphoproliferative disorders. However, Brichacek et al ${ }^{26}$ have reported interesting results in their study of $\mathbf{1 5}$ specimens derived from patients with fatal infectious mononucleosis or XLP. In this series, rearrangement of the c-myc gene was found in eight of the tissues. In addition to this, rearrangements of immunoglobulin heavy chain genes were also found, indicating the presence of clonal populations of cells. The implication is that monoclonal proliferations of B cells containing a c-myc rearrangement were found in some of these cases. What makes this tentative conclusion even more intriguing is the fact that all patients expired within 1 to 20 weeks following onset of symptoms. Thus, fatal infectious mononucleosis may be associated with the rapid emergence of monoclonal B-cell populations and oncogene abnormalities. (Rapid development of monoclonal EBV-associated tumor has also been described in the setting of congenital immunodeficiency. No evaluation of c-myc was reported from this case).$^{29}$

Locker et al. have looked for c-myc rearrangements in a number of our cases of PTLDs from the Pittsburgh series (manuscript in preparation). Positive results were found in two of 42 specimens analyzed. Both cases had a monomorphic histology and were monoclonal. One tumor was present in an ascites form, studding the peritoneum and causing the death of the patient. The other tumor, which appeared to begin in the ileum, was stubbornly recurrent, did not respond to reduced immunosuppression, and finally required chemotherapeutic intervention for eradication.

A caveat in the interpretation of such studies is the recent observation that the product of the c-myc gene may be abnormally elevated in the absence of rearrangement or amplification of the gene. ${ }^{82}$ Thus, these DNA studies may not be capable of detecting all cases in which the c-myc protein plays a role. Nevertheless, it is likely that further investigations in this area will yield results of both theoretical and practical importance. 
Hypothesis for Pathogenesis of Posttransplant Lymphoproliferative Disorder

Our hypothesis for the pathogenesis of PTLD is an extension of the hypothesis of Bird $^{15}$ for the case of lymphomas arising in transplant recipients and is similar in large part to the sequences of events postulated by Purtilo ${ }^{201,202}$ for the case of lymphomas in XLP patients, by Filipovich et al.$^{85}$ for lymphomas in naturally occurring immunodeficiencies, by Klein ${ }^{144}$ for the evolution of Burkitt's lymphoma, and by Cooper ${ }^{53,54}$ for the development of non-Hodgkin's lymphomas.

The dual basis for the production of PTLDs in immunosuppressed patients is (1) imbalance in host control of EBV-driven lymphoproliferation and (2) events conducive to tumor progression occurring on a stochastic basis within proliferating lymphocytes.

A simplified schema is depicted in Figure 7. In normal as well as in immunosuppressed individuals, EBV infection causes a polyclonal proliferation of B lymphocytes. This proliferation is normally controlled in the host by a variety of means, including the induction of B-cell maturation and T-cell cytotoxic effects. If these mechanisms do not occur, the polyclonal proliferation may continue in an uncontrolled fashion and lead to the death of the host.

In transplant patients, many cases of EBV infection are apparently controlled without incident. Rare cases show uncontrolled proliferation leading to a rapidly disseminated proliferation of lymphocytes in most organs. If the polyclonal proliferation is only partially controlled, a "polyclonal PTLD" may result. The continuing proliferation of these cells favors a clonal selection process allowing overgrowth of a dominant clone. This dominant clone may emerge due to external forces, or it may arise as a result of mutations within the cell itself. The events may be considered to represent "tumor progression" in either case.

According to the rules of tumor progression, (a) the cells will become more malignant with time and (b) independent tumors will progress independently. Therefore, although the events can be unified under the general heading of tumor progression, we may also expect multiple branch points in this process, resulting in divergent tumor clones with varying degrees of "malignancy."

What are the implications of this model in the individual patient? Obviously, events favoring EBV lymphoproliferation over host control mechanisms will favor the development of either fatal infectious mononucleosis or PTLD. Primary versus secondary EBV infection, overimmunosuppression, and constitutional host immune defects likely contribute to this process. Reduction of immunosuppression, acyclovir, and even surgical resection would tend to counterbalance these forces.

With the emergence of a monoclonal lesion, the battle shifts from 
5 (inadequate host response: $\rightarrow$ Dissemination)

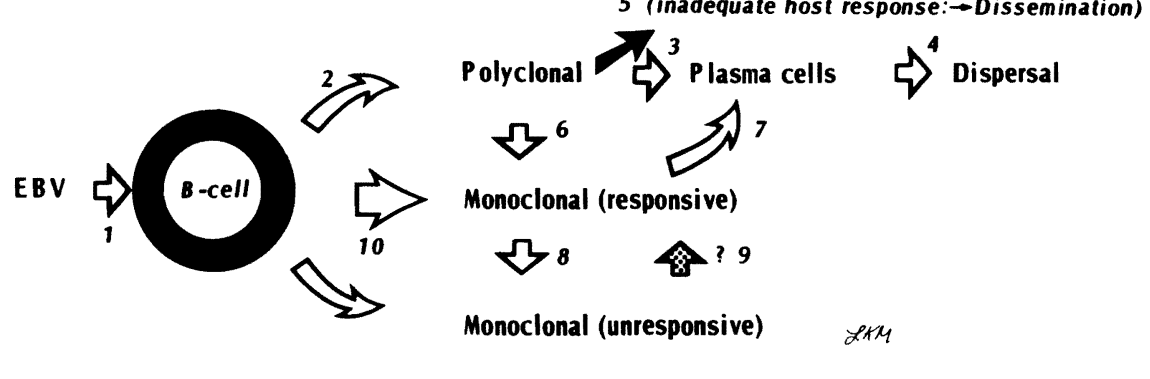

\section{FIG 7.}

Hypothetical pathogenetic sequence of PTLD development. Epstein-Barr virus infects B lymphocytes (1), which proliferate in a polyclonal fashion (2). Early in the course of infection (i.e., infectious mononucleosis), the B-cells mature into plasma cells (3). These disappear from the circulation, and residual B-lymphocytes undergo "dispersal" (4) by a variety of host effector mechanisms, most notably by the actions of cytotoxic T lymphocytes. In a minority of patients unable to mount an effective host response (e.g., XLP patients), rapid dissemination may occur (5), leading to fatal polyclonal infectious mononucleosis. Both of these pathways are also operative in transplant recipients. In addition, the polyclonal proliferation may undergo a process of clonal selection leading to a monoclonal proliferation (6). Some monoclonal proliferations may represent minimally deviant populations, since they are still responsive to host control mechanisms following a reduction of immunosuppression. The host probably controls the tumors by a reestablishment of the mechanisms normally operative in the control of infectious mononucleosis (7). With further tumor progression (8), the tumor may become unresponsive to reestablished host control mechanisms. Unresponsive monoclonal tumors may also conceivably arise directly from a polyclonal proliferation without going through an intermediary responsive phase (not shown). It is unknown whether further manipulations, for example, the induction of EBV membrane antigens on these cells, may make some of these tumors once again responsive to host control mechanisms (9). ${ }^{141}, 146$ The progression of polyclonal to monoclonal appears to occur over a long period in conventionally immunosuppressed patients. However, in CsA immunosuppressed patients, both polyclonal and monoclonal tumors occur rapidly after transplantation. This suggests either that the polyclonal to monoclonal progression is rapid, or that both responsive and nonresponsive monoclonal tumors may arise de novo following EBV infection (10).

the host:virus struggle to the host:tumor interaction. The virulence of the particular tumor clone may play a decisive role from this point on. Unfortunately, it is difficult to make any a priori assessment as to the degree of malignancy, because of the multiplicity of cellular events that may occur and the limited number of patients with these conditions. In our experience, however, tumors that are polymorphic and polyclonal or polymorphic and monoclonal are more likely to be responsive to reestablished host immune control mechanisms than are tumors that are monomorphic and monoclonal. We have not yet observed any tumors that are monomorphic and polyclonal. These histopathologic distinctions are not absolute, 
however, indicating the necessity for a clinical trial of reduced immunosuppression and medical/surgical support in order to evaluate the behavior of the tumor(s).

The responsiveness of a monoclonal tumor suggests that $(1)$ the tumor has a competitive growth advantage over other clones but is not yet fully malignant, or (2) the tumor is fully malignant but expresses strong (viral?) antigens. Either of these possibilities would render the tumor susceptible to host immune defenses. With further tumor progression, the neoplasm escapes beyond the reach of the host immune controls.

It has generally been assumed that a substantial period of time was needed for enough events to occur in order to allow the emergence of a monoclonal proliferation from a polyclonal background. In the case of non-CsA regimens, monoclonal tumors do not occur until years after transplant whereas polyclonal proliferations occur much earlier. In the case of CsA immunosuppression, however, monoclonal proliferation may be seen very early after transplant. This suggests either a de novo monoclonal proliferation or the rapid emergence of a monoclonal tumor from a polyclonal background.

Based on our hypothesis of the pathogenesis of PTLD, we conclude:

1. Prevention of EBV infection would eliminate this form of PTLD.

2. Polyclonal proliferations are technically benign lesions. Thus, control of these lesions can be effected by eliminating the stimulus for proliferation or by augmenting or correcting the host immune control mechanisms. The use of acyclovir and reduced immunosuppression addresses both of these aims in the absence of a constitutional host defect.

3. Additional influences, beyond EBV stimulation, cause the emergence of monoclonal tumors. Thus, antiviral therapy alone is ineffective in this form of disease.

4. Some monoclonal tumors will, and some will not, respond to reestablished host immune control. The response is dependent on the integrity of the host immune response, the degree of tumor progression toward malignancy, and the relative rates of tumor growth and tumor destruction. Therefore, response to therapy should be closely followed.

5. Independent tumors will progress toward malignancy independently. Therefore all tumors within a patient should be followed for response to therapy.

In summary, this multistep scenario suggests that a graded form of therapeutic intervention be employed, beginning with reduced immunosuppression, administration of acyclovir, and control of local complications. These measures are usually sufficient. Only those 
cases that do not respond to such measures should be considered for more aggressive antilymphoma therapies. These points will be more fully presented under Clinical Considerations and Case Presentations, below.

\section{CLINICAL CONSIDERATIONS}

\section{PATIENT POPULATION}

As of March 1987, 43 patients in the combined Pittsburgh-Denver transplant series were diagnosed as having PTLD. These included 29 males and 14 females ranging in age from 1 to 62 years, with a mean age of 23 years. The 43 patients accounted for $1.7 \%$ of the total transplant population at that time. There were 23 liver, 12 kidney, 5 heart, and 3 heart/lung transplant patients in the series. This constituted a relative frequency for PTLD of $2.2 \%, 1.0 \%, 1.8 \%$, and $4.6 \%$ in the respective organ allograft subpopulations. Earlier reports from our series $^{244}$ initially suggested a much higher incidence in heart and heart/lung recipients, an experience which has not been supported with time. ${ }^{165}$ Despite this change, the overall incidence of PTLD at our institution has not changed significantly from earlier reports. ${ }^{215}$

Forty-one of the 43 patients had received initial immunosuppression with various CsA-prednisone-containing regimens. The remaining two patients received CsA later in their course. In addition to these drugs, 9 patients received OKT3, 4 received azathioprine, 3 received ALG, 3 received azathioprine and OKT3, and 1 underwent thoracic duct drainage. Tissue matching was on a random basis, especially for nonrenal donors.

\section{TIME OF ONSET OF POSTTRANSPLANT LYMPHOPROLIFERATIVE DISORDER}

It has been our experience ${ }^{165}$ and the observation of others ${ }^{12,185,193}$ that PTLDs occurring in association with CsA-containing regimens occur more rapidly after transplantation than do those PTLDs that occur in non-CsA-immunosuppressed patients. The reason for this is unknown. However, it is interesting to speculate that it may be related to the interaction between drug and host immune response. Perhaps CsA is a more potent inhibitor of the host anti-EBV immunosurveillance system. In that case, it may allow more florid outgrowth of PTLDs at an earlier and more reversible stage of development. Conversely, standard immunosuppressive regimens may allow a subclinical, "smoldering" lymphoproliferation over time. This scenario would help account for the observation that early PTLDs occurring in non-CsA immunosuppressed patients resemble infectious mononucleosis but that late PTLDs in these patients are solid tu- 
mors. Such a temporal distinction is not seen in CsA-immunosuppressed patients.

The majority of the 41 patients in our series who initially received CsA-containing regimens had tumor onset within 1 year after transplant, with a median onset time of 4.4 months, and a range of $0.7-$ 26.2 months. Two other patients in our series who were initially immunosuppressed with azathioprine-containing regimens had tumor onset times of 68 and 162 months following transplant. Some patients initially immunosuppressed with CsA were also given supplemental OKT3 or ALG. No difference in PTLD onset time was observed in these patients compared to the CsA patients as a whole. Likewise, those patients who initially received CsA regimens and were later given azathioprine had a tumor onset time identical to the general CsA population.

In the Minnesota series an infectious mononucleosis-like presentation was seen at a mean time of 9 months after transplant. Presentation with solid tumor occurred at a mean onset time of 5.3 years following transplant. ${ }^{108}$ All patients except one in this series were on non-CsA-containing regimens. The one patient who received CsA had a mononucleosis-like syndrome at 16 months after transplant.

Perhaps the most graphic display of the effect that the immunosuppressive regimen exerts on the onset time to tumor comes from the Stanford series. Of 10 patients with PTLD, five received conventional immunosuppression and five received CsA-containing regimens. In the first group, the number of days between transplant and tumor were $935,1,335,1,487,1,514$, and 2,575 , respectively. ${ }^{50}$ In the second group, these intervals were 99, 101, 122, 151, and 187 days.

\section{CLINICAL PRESENTATION}

The presenting symptoms are dependent upon the localization of the PTLD, the severity of the disease, and the existence of concurrent infection. Some patients may be entirely asymptomatic, indicating the need for the physician to maintain a high index of suspicion.

As has been observed in other transplant series, a significant proportion of patients present with signs and symptoms resembling infectious mononucleosis. In this group, rapid progression of the proliferation may lead to early complications such as respiratory compromise due to tonsillar hypertrophy. Emergency tracheostomy may be required in such cases. Although the most dramatic symptomatology is referable to the head and neck region in the form of tonsillar enlargement, cervical adenopathy, and salivary gland enlargement (due to intrasalivary gland lymphoproliferation), the disorder often extends beyond this region. Contemporaneous systemic manifestations may be subclinical or may be as innocuous as mild lymphadenopathy. Conversely, some patients presenting with pre- 
dominant head and neck disease may also harbor other, unsuspected tumors at presentation.

In our series, approximately $25 \%$ of patients presented with gastrointestinal manifestations. These included acute abdomen due to perforation of a hollow viscus, pain, obstruction, or gastrointestinal hemorrhage. Gastrointestinal disease has not been prominent in other transplant series utilizing conventional immunosuppression, ${ }^{185}$ but has been noted with Burkitt's lymphoma, AIDS lymphomas, and in lymphoid tumors of XLP patients. The reasons behind these distribution differences are not apparent.

A third category of clinical presentation constitutes a variable complex of signs and symptoms, having in common a predominant involvement of a single or few organs. Thus, individual patients may present with lung lesions, renal mass, prostatic obstruction, or other indicators of tumor localization. Frequently this process first manifests in the allograft organ, where it may mimic rejection. In such cases, biopsy diagnosis may be the first indicator of PTLD.

In non-CsA immunosuppressed patients, localization of PTLD to the CNS elicits the presenting symptomatology in a significant number of cases. ${ }^{39}$ Central nervous system involvement appears rare in CsA-immunosuppressed patients. ${ }^{185}$ In our experience, this type of involvement is seen only in the face of widely disseminated disease. However, in the Minnesota experience, cited above, $14 \%$ of patients had PTLD confined to the CNS. ${ }^{108}$ If the increased incidence of CNS lymphomas in conventionally immunosuppressed patients were explainable wholly by the concept of the brain as an "immunologically privileged site," ${ }^{\prime \prime 3}$ then one must inquire why a similar localization of tumor is not seen in patients receiving CsA.

Occasional case reports exist demonstrating the emergence of PTLD at the site of antilymphocyte globulin injection. ${ }^{55,70}$ Interestingly, in one case, ${ }^{70}$ a nodule that formed at the site of injection remained at a small and stable size for $\mathbf{1 0}$ months, at which time it rapidly enlarged and was diagnosed as a reticulum cell sarcoma.

Rarely, patients present with fulminant disease and multiorgan involvement. The response of these patients to all therapies appears poor. It is not known whether this represents an extremely advanced tumor, delay in diagnosis, or a profound defect in the host immune system. Further studies are needed to clarify this point.

In six of our 44 patients, the diagnosis of PTLD was first made at autopsy. These patients generally had multifocal disease in association with other, severe opportunistic infections.

Touraine et al. ${ }^{259,260}$ have observed that PTLD may be accompanied in its early stages by abnormal serum electrophoretic patterns detectable by isoelectric focusing. This approach may form the basis of a means to screen for PTLD, especially in the first year after transplant in CsA-immunosuppressed patients. However, the high false- 
positive rate of this technique ${ }^{259}$ indicates that it needs to be combined with a test of greater specificity.

\section{ASSOCLATED INFECTIONS}

In keeping with the proposed etiologic cofactors of PTLD, serologic evidence of EBV infection was observed in 40 of the 43 patients in our series. ${ }^{165}$ Primary EBV infections outnumbered reactivation infections by a ratio of 2.3:1. Ninety-four percent of patients under the age of 18 had primary, rather than reactivation, infection. Ho et al. ${ }^{121}$ have emphasized the importance of primary EBV infection in this disorder. Our results are similar to those in other previously published studies in which the overwhelming majority of patients had evidence of EBV infection. ${ }^{110,111}$ The absence of EBV in some patients raises the possibility of other unknown predisposing factors.

The presence of EBV within the tumor cells has also been repeatedly documented by the use of immunofluorescence methods to detect EBNA or by the demonstration of the EBV genome within DNA extracted from the lesions. ${ }^{121}$ Again, rare cases are negative for EBV by these techniques. ${ }^{721}$

Over half of the patient population had other concurrent infections at the time of PTLD diagnosis. Pneumocystis carinii, Herpes simplex, and cytomegalovirus were the most frequently identified pathogens in these patients. This lends further support to the concept that many of these patients are functionally overimmunosuppressed. These additional infections may also serve to cause further immunosuppression in the patients.

\section{CLINICAL COURSE AND THERAPY}

Twenty seven of the 43 patients in our series have demonstrated resolution of PTLD ${ }^{165}$ (Nalesnik et al., submitted). In 10 of these 27 patients, reduction of immunosuppression (with administration of intravenous acyclovir in two cases) constituted the sole form of therapy. Surgical intervention was limited to biopsy for diagnostic purposes. Evidence of monoclonality was observed in four of these $\mathbf{1 0}$ patients. Follow-up time ranges from 13 to 59 months in this group.

The other 17 patients with resolution of PTLD had more aggressive surgical intervention due to either the size of the tumor or the presence of tumor-associated complications. Evidence of monoclonality was observed in seven of these 17 patients. Five of the 17 patients, including three with monoclonal disease, received additional chemotherapy or radiotherapy, or both. In retrospect, this intervention may have been unnecessary in some cases. One of these patients, however, had multiple recurrences of tumor following resec- 
tions and discontinuation of immunosuppression. It was in this setting that chemotherapy was instituted. This case is presented in detail below (case 6).

No response to therapy was seen in five of the 44 patients. These patients all had evidence of monoclonal tumors that were widespread. One patient developed invasive aspergillosis following chemotherapy. Another had disseminated cryptococcosis, underscoring the severity of the immune defects. These patients often had delays in diagnosis and in therapeutic intervention, contributing to a complicated course. Nevertheless, these complications do not negate the fact that these malignancies led to the deaths of these individuals.

In five patients, it is not possible to come to an unequivocal conclusion regarding tumor behavior. Two of these five appear to have had partially to nearly complete resolution of tumor but expired of related complications. In the other three patients, no significant therapeutic response was possible, and they expired shortly after diagnosis. The difficulty in using death as an endpoint in concluding that a tumor is not responsive to therapy is considered in the discussion of cases 4 and 7 .

Tumor was diagnosed at autopsy in six patients. All had evidence of other severe and multiple infections at that time.

Several clinical features suggest a more favorable prognosis, on the basis of our patient series. In our experience, patients with tumors predominantly involving the gastrointestinal tract have an excellent prognosis with appropriate therapy. Over $80 \%$ of our patients with this form of disease are alive from 3 to 7 years after diagnosis. These tumors, usually multiple, can rapidly cause perforation, obstruction, or intussusception, all of which must be surgically relieved.

A good prognosis is also associated with a predominantly head and neck presentation of PTLD (excluding CNS disease). These cases probably include a number of cases of infectious mononucleosis with minimal progression toward a full-blown PTLD. But some monoclonal lesions also may be seen in this area as well. Eleven of thirteen patients with this form of presentation have undergone resolution of their disease.

A disseminated presentation of disease, often in association with other infections, was a poor prognostic indicator. These patients expired within a short period of time regardless of the form of therapy employed. Fortunately, this manner of presentation is uncommon and may be made even rarer by a high index of suspicion on the part of the physician, which will allow earlier diagnosis and treatment of lesions before they progress to this stage.

Conversely, limited organ involvement is often a treatable disease. Cases predominantly involving the lung or kidney appear to respond to conservative management as outlined above. Isolated CNS involve- 
ment was not seen in our series. However, in reports from other centers, this form of disease is associated with a poor prognosis.

Pathologic studies may give additional useful information but must be interpreted in conjunction with the extent of disease. Polymorphic and polyclonal tumors are more likely to undergo regression than are monomorphic and monoclonal tumors, but the distinction is not absolute. More sensitive indicators such as the intensity of the rearranged monoclonal band on Southern blot and the presence of myc-oncogene rearrangement may allow better prognostication in the future (Locker $J$ et al., unpublished report). For the present, the response of tumor to a clinical trial of reduced immunosuppression and administration of acyclovir remains a favored approach in planning subsequent management.

Concern has been expressed over the reduction of immunosuppression in recipients of allografts other than kidneys, in which supportive therapy may not be available should organ rejection occur. In our opinion, PTLD is a manifestation of functional overimmunosuppression, regardless of the absolute amount of drug given to the patient. In accordance with this, we have observed that a reduction of CsA by as much as $50 \%$ may often be employed with only a minimal to mild risk of organ rejection.

Therefore, our initial therapeutic approach is to reduce immunosuppressive medications to about $50 \%$ of the baseline dose following the diagnosis of PTLD. The patient is then observed clinically for (1) evidence of regression of PTLD and (2) the possible emergence of allograft rejection. If necessary, we will further reduce or even discontinue immunosuppession depending upon the clinical course.

In following allograft status by biopsy, care must be taken not to confuse rejection with PTLD. PTLD generally shows immunoblastic differentiation with plasmacytoid features, and large atypical cells can be seen on occasion. These features are lacking in the rejection response, which localizes to specific structures in specific organs. Simultaneously, the fate of the residual PTLD must be followed. This is best done radiologically, in addition to monitoring the patient clinically for the appearance of acute tumor complications. Clear evidence of tumor regression should be seen within 2 weeks and usually is seen within a few days. If no changes are seen by this time period, it is still possible that cure may be effected by resection of a localized residual tumor. If the tumor continues to grow during this interval, these measures may prove inadequate and chemotherapy should be considered. However, the use of chemotherapy as a first line measure in these immunologically compromised patients is to be strongly discouraged. Our experience, as well as that of others, ${ }^{40}$ shows that death from sepsis is a likely outcome in this setting. No center has successfully managed a large series of cases by the use of chemotherapy. In this regard we are no exception. Nevertheless, we 
feel that chemotherapeutic intervention must take into consideration (1) the initial immunosuppressed condition of the patient and (2) the often striking response of EBV-associated Burkitt's lymphoma to minimally to moderately aggressive antilymphoma treatment.

Assuming that the patient has clinically responded to a therapeutic regimen including reduced immunosuppression, a thorough evaluation for occult or recurrent tumors should be performed at intervals. Following remission of PTLD we generally resume the patient on initial or slightly reduced levels of immunosuppression.

The similarity of our approach to that of others is evident on the basis of scattered case reports. In one example, Bia and Flye ${ }^{13}$ documented regression of pulmonary tumor nodules that arose in association with a multicentric, monoclonal intestinal PTLD in a renal transplant recipient immunosuppressed with CsA and prednisone. The intestinal tumor was resected, but the lung nodules were not excised. Monoclonality of the intestinal tumor was diagnosed by immunoglobulin gene rearrangement studies. CsA was discontinued completely, resulting in allograft rejection. The lung nodules disappeared totally and there is no evidence of recurrence to date, 43 months after initial diagnosis (Bia, personal communication, 1987).

Hanto et al. ${ }^{108,109}$ stress the efficacy of acyclovir in alleviating PTLD classifed as polymorphic diffuse B-cell hyperplasia in which a polyclonal pattern is documented. In the presence of PTLD diagnosed as polymorphic B-cell lymphoma, or with documentation of a monoclonal component, they carefully increase their level of intervention, including a reduction of immunosuppression. We feel that this is a reasonable approach but prefer to reduce immunosuppression as a first-line measure, usually with the addition of acyclovir. In this manner we do not risk allowing the continued proliferation of latently infected cells. ${ }^{109,237}$ The price to be paid is the increased risk of organ rejection. However, we must emphasize that it is possible to titrate the reduction of immunosuppression in order to minimize and abort this risk of rejection. Incidently, we agree with Hanto ${ }^{108,109}$ that organ rejection must occasionally be allowed to proceed in order to gain the upper hand on the PTLD. An uncontrolled lymphoproliferation in this setting will otherwise quickly cause the death of the patient.

Bird and Britton, in an elegant review of EBV and lymphoma, had previously recommended a reduction of immunosuppression for treatment of polyclonal disease. They also raised the possibility that some monoclonal tumors may and some may not respond to this intervention. ${ }^{15}$

The 1978 report of Geis et al. is of interest, since it apparently describes regression in five cases of PTLD with no reduction of immunosuppression and with no antitumor therapy. ${ }^{96}$ However, tumors in all of these patients arose in response to a particular lot of 
anti-thymocyte globulin. When this drug was discontinued, therefore, a de facto reduction of immunosuppression did indeed occur.

\section{SPECIMEN PROCESSING IN SUSPECTED CASES OF POSTTRANSPLANT LYMPHOPROLIFERATIVE DISORDERS}

The two purposes of surgical intervention in cases of suspected PTLD are (1) diagnosis and (2) management of complications (e.g., perforated bowel, respiratory obstruction). Often both purposes coincide.

The full diagnosis of PTLD is dependent on a variety of histologic, immunophenotypic, virologic, and immunogenotypic techniques. Much information can be obtained from the histology alone. We do not, however, encourage the use of frozen sections for several reasons: (1) a presumptive diagnosis of PTLD can usually be made either by the clinical presentation or by the appearance of mass lesions at operation; (2) frozen sections destroy the fine histologic details of the tissue and in most cases will only support the surgical suspicion without offering any additional useful information (an erroneous overdiagnosis of malignant lymphoma is especially likely in this setting); (3) tissue may be more profitably divided for other studies.

The surgeon should provide the pathologist with as much tissue as possible to perform adequate studies. Though trite, this dictum is of especial importance in this case. In cases of multiple tumors it is useful to provide specimens from as many tumors as possible. Due to the independence of tumor progression, separate lesions may have reached different levels of malignant transformation.

The specimen should be provided fresh and sterile to the pathologist as soon as possible after resection. It should be kept moist with a small amount of sterile saline or, if at all possible, tissue culture fluid. Separate specimens should be submitted for culture, including viral culture.

Once received from the surgeon, the specimen is further divided into aliquots by the pathologist. A small portion of sterile tissue should immediately be submitted for karyotypic analysis. There are several options of tissue processing for clonal and virologic analysis. Clonal analysis may be performed on frozen tissue sections or on cell suspensions by immunocytochemistry. Alternatively, tissue may be set aside for immunogenotypic analysis of clonality. We prefer a combination of the two methods. Immunohistochemical analysis gives rapid results that may be sufficient in a number of cases, while immunoglobulin gene rearrangement analysis is a more sensitive indicator of clonal composition. The latter technique, if available, usually has a prolonged turnaround time. 
Alternative techniques are also available for demonstration of EBV within the tumor specimen. Immunohistochemical staining for Epstein-Barr nuclear antigen (EBNA) may be performed on touch preparations or, more satisfactorily, on frozen tissue sections. Detection of the EBV genome by DNA hybridization requires purification of DNA from the tumor.

In practice, we usually freeze a portion of the specimen for the various immunohistochemical analyses and separately freeze a portion of tissue for purification of DNA. Both clonal and virologic studies can be performed on both specimens if necessary.

Following the allotment of tissue for these purposes, a portion of these is submitted for routine light microscopic analysis. We usually submit a minute fragment of tumor for electron microscopy but have not found this to be a useful adjunct to date.

Other studies are dependent on the interests of the investigators.

The results from routine histology frequently establish the diagnosis and determine whether the lesion is monomorphic or polymorphic. Analysis of EBNA determines the presence or absence of EBV within the lesion. Immunohistochemical analysis of clonality may show a clear-cut monoclonal lesion or a likely polyclonal lesion, or frequently may be ambiguous or negative.

In the presence of a positive EBNA stain, the genomic analysis of EBV DNA will almost always confirm the result. However, it is not uncommon for an EBNA-negative tumor to subsequently show positive results for the presence of EBV by the technique of EBV DNA hybridization. This is because of technical difficulties, especially antigen lability, inherent in the performance of the EBNA test.

Likewise, it has been our experience that a clear-cut monoclonal determination on immunohistochemistry will be confirmed by DNA studies. The surgeon should not be too surprised, however, if a tumor initially thought to be polyclonal later shows a monoclonal component on the basis of Southern blot studies. By that time, however, the tumor will frequently have declared its clinical behavior one way or the other.

\section{CASE PRESENTATIONS}

Case 1.-This 21/2-year-old female with biliary atresia and a history of failed Kasai procedure underwent orthotopic liver transplantation on May 10, 1984. Her postoperative course is shown in Figure 8. Recovery was prolonged due to ischemic liver damage; however the patient was discharged 6 weeks after transplant in excellent condition. In mid-July she developed fever, malaise, cervical and inguinal adenopathy, and rapid tonsillar enlargement that required emergency tracheotomy due to upper respiratory impairment. At that time, a tonsillectomy and inguinal lymph node biopsy were performed. The tonsils demonstrated a diffuse, polymorphic prolifer- 


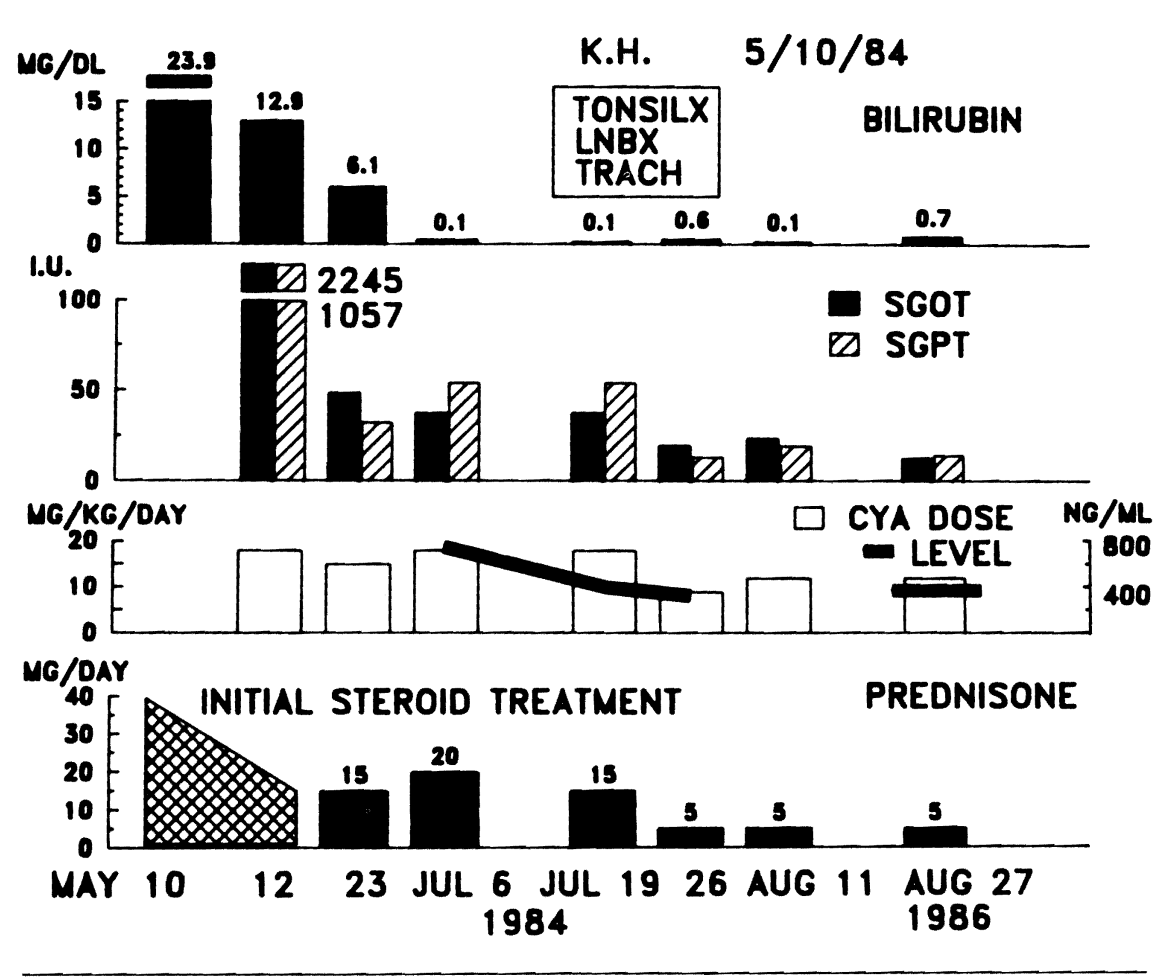

FIG 8.

Case 1: Clinical course of a $2 \frac{1}{2} 2$-year-old female liver recipient who developed PTLD resulting in acute upper airway obstruction and requiring tracheostomy, tonsillectomy, and reduction of immunosuppression. (From Makowka L, Nalesnik MA, Stieber A, et al: Control of post-transplant lymphoproliferative disorders and Kaposi's sarcoma by modulation of immunosuppression, in Good RA, Lindenlaub E (eds): Symposia Medica Hoechst: The Nature, Cellular, and Biochemical Basis and Management of Immunodeficiencies. Stuttgart-New York, FK Schattauer Verlag, 1987, pp 567-624. Reproduced with permission.)

ation of lymphocytes in all stages of development in association with large areas of necrosis and with large, atypical Reed-Sternberg-like cells. A diffuse plasmacytic proliferation with retention of underlying architecture was observed in the inguinal node. A diagnosis of PTLD was given, and the process was judged polyclonal on the basis of immunoperoxidase stains for cytoplasmic immunoglobulins as well as immunoglobulin gene rearrangement studies.

The patient's CsA was reduced from 15.5 to $7.75 \mathrm{mg} / \mathrm{kg} /$ day and prednisone from 10 to $5 \mathrm{mg} /$ day, and she received a full intravenous course of acyclovir. She recovered promptly and is currently well 40 months later, with normal hepatic function.

Comment.-The rapid growth of the tonsils leading to respiratory embarrassment serves to underscore the clinical severity of the dis- 
ease. In this case, pathologic documentation of the "benign" nature of the proliferation came much later and did not influence the therapeutic intervention, although it did serve to provide a logical basis for the resolution of disease. It is important to recall that acute airway obstruction may also be seen as a rare complication of otherwise typical infectious mononucleosis. ${ }^{160}$ The preferred treatment in that circumstance consists of high dose steroids. Concern has been expressed over the long-term effect of this treatment. ${ }^{160}$ In the immunosuppressed patient we would express similar concerns, especially since this therapy may further exacerbate the underlying host immune imbalance. The concurrence of tonsillectomy, reduction of immunosuppression, and acyclovir does not allow a conclusion as to which maneuvers might have been sufficient and which superfluous. However, in practice, judicious operation coupled with reduction of immunosuppression, with or without acyclovir, has become the preferred approach at our institution.

Case 2.-This 3-year-old male received a liver transplant on August 7, 1984, for treatment of liver failure due to homozygous $\alpha_{1}$-antitrypsin deficiency. Eleven months later he was readmitted to Children's Hospital following a 5-day history of fever, decreased appetite, vague abdominal pain, and cervical adenopathy. Tonsils were also enlarged. A biopsy of a $2-\mathrm{cm}$ left cervical node showed a minimally polymorphic proliferation with plasmacytic differentiation. Bands of necrosis were observed. A diagnosis of PTLD was given. Immunoperoxidase studies were consistent with a polyclonal proliferation. However, later immunoglobulin gene rearrangement studies showed a monoclonal component to the proliferation.

CsA was reduced from 19 to $12 \mathrm{mg} / \mathrm{kg} /$ day and prednisone from 7.5 to 5 $\mathrm{mg} / \mathrm{kg} /$ day. No acyclovir was given. Adenopathy and tonsillar enlargement resolved, and liver function tests remained normal. The patient remains well without evidence of disease 28 months after diagnosis.

Comment.-This case is of interest because of the presensce of an unsuspected monoclonal component to the PTLD on the basis of DNA studies. Despite this, the disease resolved in association with a reduction of immunosuppression. It is likely that "tumor progression" had evolved to the point that a clone of cells had emerged from a nonclonal background. The presence of numerous plasma cells with a minimally polymorphic appearance suggests that the tumor cells retained the capacity to mature. Conversely, a minor and less differentiated component of the proliferation may not have been appreciated on pathologic examination. Although this patient's disease completely resolved with appropriate therapy, current hypotheses suggest that, if this proliferation had been allowed to continue, a more malignant cell clone refractory to host control mechanisms would have emerged. Acyclovir has the potential to inhibit polyclonal proliferations due to EBV but has no effect on the latent virus ${ }^{52}$ often seen with clonal proliferations. Therefore the ef- 
ficacy of this drug as the sole therapeutic agent in this case, although problematic, appears theoretically inferior to either a reduction of immunosuppression or a combination of acyclovir and reduced immunosuppression. ${ }^{77}$

Case 3.-The patient was a 20-year-old male who received an orthotopic liver transplant on January 12, 1982, for treatment of liver failure secondary to chronic active hepatitis. Retrospective serologic studies showed a primary EBV infection between March 12 and August 16, 1982. He presented with cervical adenopathy on August 24, 1982. Pathologic sections were initially interpreted as nonspecific reactive hyperplasia. The appearance was that of a minimally polymorphic, diffuse, plasma cell proliferation with no necrosis and with retention of underlying lymph node architecture. Epstein-Barr virus DNA was detected in the tissue as well. (Thus the lesion qualifies as an early form of PTLD).

No treatment was administered. He presented elsewhere in November with gastroenteritis and was again discharged with no specific therapy. On December 4, 1982, he re-presented elsewhere with a 1-week history of severe pharyngitis and odynophagia. He was treated for a staphylococcal abscess but remained febrile, with a necrotizing tonsillitis and bilateral cervical lymphadenopathy. The patient sustained irreversible laryngospasm during a laryngoscopic procedure and expired on December 14, 1982. At autopsy, polymorphic PTLD with necrosis was seen in the tonsils. Additionally, plasmacytoid infiltrates were seen in several organs and in the colon, where they were associated with hemorrhagic ulcers. Clonal studies were inconclusive.

Comment.-In contrast to the first two cases, sustained lymphoproliferation with clinical consequences was seen in this patient who was maintained on full immunosuppression. The histologic changes at autopsy appeared more advanced than those seen in the original lymph node biopsy. The infiltration of organs and presence of colonic ulcers indicate the systemic nature of this illness. Death in this case does not appear to be an indicator of malignancy, since it followed a diagnostic procedure. It is likely that the patient's disease was still reversible at this point had the proper measures been taken. Of course, recognition of the process at first presentation would have led to control of the disease at the outset.

Case 4.-This 16-year-old male with Laurence-Moon-Biedl syndrome underwent a renal transplant on December 12, 1982, for end-stage renal disease secondary to this disorder (Fig 9). He was discharged in good condition 4 weeks after transplant. On March 26, 1983, he re-presented with severe guaiac-positive diarrhea. While in the hospital he developed an acute abdomen secondary to a perforated hollow viscus. At laparotomy multiple tumors, involving the ileum and colon, were found. The gross appearance of tumors was similar to that shown in Figure 2. Three separate perforations were discovered. Pathologic examination led to the diagnosis of PTLD. Immunoperoxidase studies of multiple tumors showed 11 of 13 to be monoclonal. One regional lymph node was also involved by tumor. It was not 
L.S. $\quad 12 / 82$

MG/DL

SB RESX

$\triangle$ CREATININE

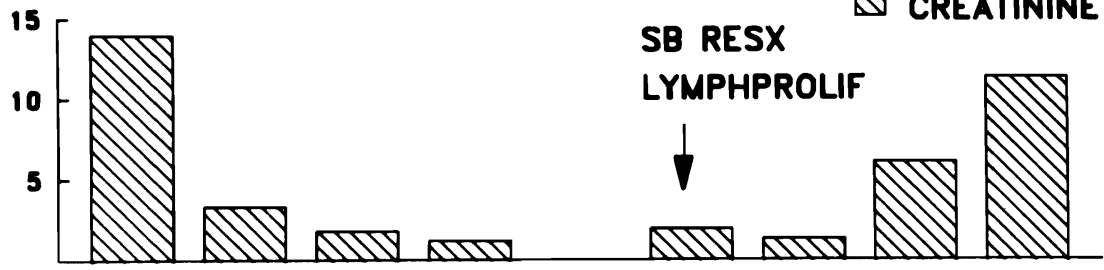

MG/KG/DAY
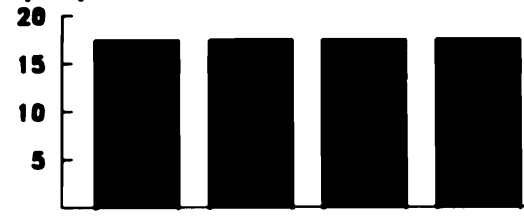

CYA

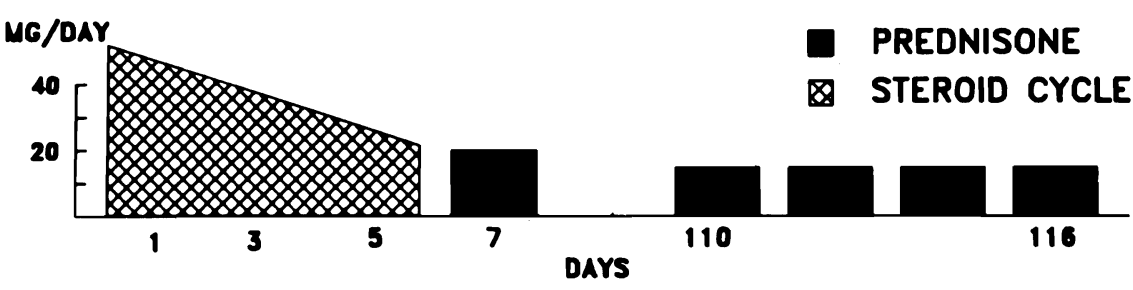

FIG 9.

Case 4. Clinical course of a 16-year-old renal transplant recipient who developed multiple small bowel PTLDs with perforation, requiring emergency resection. In this 1982 case, immunosuppression was completely discontinued, and graft rejection was allowed to proceed. A second resection to remove remaining tumors revealed marked regression of previously observed masses. (From Makowka L, Nalesnik MA, Stieber A, et al: Control of post-transplant lymphoproliferative disorders and Kaposi's sarcoma by modulation of immunosuppression, in Good RA, Lindenlaub E (eds): Symposia Medica Hoechst: The Nature, Cellular, and Biochemical Basis and Management of Immunodeficiencies. StuttgartNew York, FK Schattauer Verlag, 1987, pp 567-624. Reproduced with permission.)

possible to remove all of the tumors at that time. Postoperatively the patient's CsA was discontinued completely and his prednisone maintained at $15 \mathrm{mg} /$ day. Five weeks later an allograft nephrectomy was performed due to intractable rejection. At that time the abdomen was again explored by the same surgical team, who noted with surprise that the tumors had either reduced in size or disappeared completely. They resected the only suspicious area, near the site of primary anastomosis (Fig 10). The pathologists were unable to reach a definitive diagnosis and termed the process "multiple nonspecific healing and chronic active ulcers." The microscopic appearance was one of mucosal epithelial regeneration and underlying fibrous repair. Scattered throughout was a moderately dense infiltrate of mature plasma cells. Immunoperoxidase studies, however, again revealed the individual lesions to be monoclonal. 


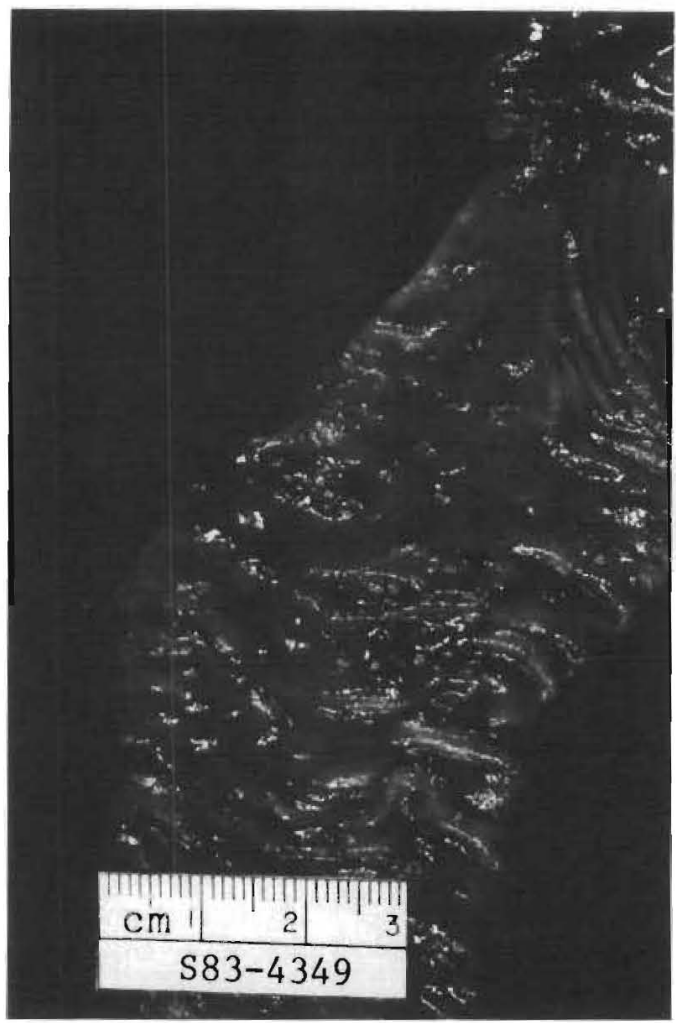

FIG 10.

Case 4. Representative remaining "tumors" of bowel resected following a period of discontinuation of immunosuppression. Other intestinal tumors were removed several weeks eartier and had a gross appearance similar to that seen in Figure 2. Prior anastomotic site is seen in the upper right-hand corner of photograph. See text for details

The patient remains well 56 months after tumor diagnosis and is currently awaiting a new kidney.

Comment.-This case is instructive in conceptualizing the basis for our approach to this disorder. It is obvious that the multiple bowel perforations could have led to death in this patient with monoclonal tumors. Had the case ended here, the diagnosis of "malignant lymphoma" would have seemed logical. However, rapid surgical intervention allowed the patient to survive this episode. Again, if all of the visible tumors had been removed, the argument could be made that this alone effected a cure. But in this case it was necessary to leave tumors behind. The clinical resolution coincided with discontinuation of CsA and rejection of the kidney allograft. Histologically, the cells had matured into plasma cells but retained their monoclonal nature. The bowel wall showed changes of tissue repair, 
indicating that the process was resolving. The available evidence suggests that the tumor cells matured into plasma cells in association with a reduction in cell mass (i.e., dispersion or destruction of cells).

One may still argue that a case for tumor cell maturation cannot be made because two different sets of tumors were sampled at two separate times. That these resolving lesions were somehow different is unlikely in light of the clinical course. In addition, clonal studies of $\mathrm{EBV}^{205}$ and immunoglobulin genes from a similar case indicate that multiple gut lesions arose from a single clone that underwent a dysplastic form of maturation, resulting in multiple separate, derivative tumors (Locker et al., unpublished observation, 1987). Yet a third case with a similar related clinical course provided several gut tumors following a period of reduced immunosuppression. In this instance one tumor showed cell maturation and a second resembled the original tumor and did not show maturation. However this second tumor was associated with extensive necrosis and contained a small lymphocytic infiltrate that may have represented a cytotoxic host immune response. It is thus likely that there are several mechanisms by which tumor regression may be effected.

From a clinical standpoint, this case illustrates the coordinated use of surgery and modulation of immunosuppression to control local complications and cause tumor regression. Because of the often explosive growth of these lesions, life-threatening disorders of this type may arise before the host can mobilize defenses against the tumors. Such complications should be handled expeditiously and are not to be construed as evidence that the lesion is intrinsically unresponsive to host immune controls.

Case 5.-A 43-year-old white male received an orthotopic liver transplant for treatment of liver failure secondary to Laennec's cirrhosis (Fig 11). His postoperative course was complicated by severe renal failure, fungemia, and multiple episodes of rejection. He eventually recovered from all of these problems and was discharged with good hepatic and renal function.

Six months after transplant, the patient was admitted elsewhere with a productive cough and dyspnea. Pneumocystis pneumonia and disseminated cytomegalovirus infection were diagnosed (Fig 12). CsA was discontinued (5.5 to $0 \mathrm{mg} / \mathrm{kg} /$ day) and prednisone was decreased from 7.5 to $5 \mathrm{mg} /$ day. Trimethoprim therapy and respiratory support were also administered. His condition improved except for the presence of a persistent nodular infiltrate in the lungs (Fig 13). An open lung biopsy was performed and revealed a monomorphous PTLD. Immunosuppression was completely discontinued, and the lesions completely disappeared. He was discharged on low maintenance immunosuppression and is alive and well with good liver function and no evidence of disease 18 months after PTLD diagnosis (Fig 14).

Comment.-Cases 4 and 5 show regression of lesions that were clinically much different from those in cases 1-3. It may be argued, 


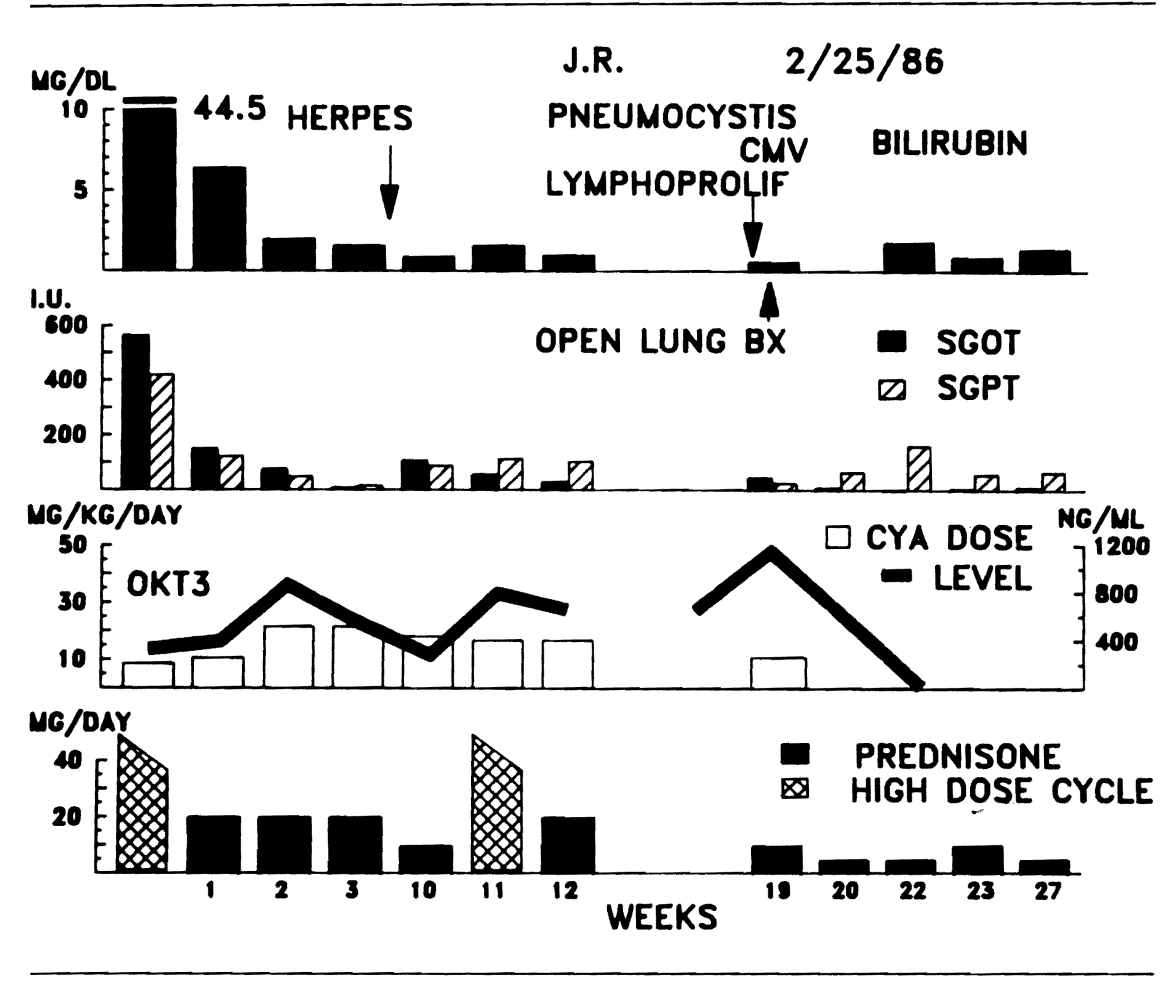

FIG 11.

Case 5. Clinical course of a 43-year-old male liver transplant recipient who presented with pneumocystis pneumonia, generalized cytomegalovirus infection, and respiratory failure. Pulmonary PTLD was diagnosed by open lung biopsy. (From Makowka L, Nalesnik MA, Stieber A, et al: Control of post-transplant lymphoproliferative disorders and Kaposi's sarcoma by modulation of immunosuppression, in Good RA, Lindenlaub E (eds): Symposia Medica Hoechst: The Nature, Cellular, and Biochemical Basis and Management of Immunodeficiencies. Stuttgart-New York, FK Schattauer Verlag, 1987, pp 567-624. Reproduced with permission.)

probably correctly, that the first several cases represent nothing more than severe infectious mononucleosis and therefore the treatment of these patients in no way applies to the treatment of transplant patients with "malignant lymphomas." Case 4 was shown to be monoclonal by immunophenotype. Case 5 is most likely monoclonal because of our finding that all monomorphic PTLDs in which clonality has been determined have been monoclonal to date. The observed regression of these lesions suggests that tumor progression of PTLDs contains an intermediate zone composed of monoclonal tumors that are still responsive to host control mechanisms. A separate case of a monomorphic and monoclonal PTLD occurring in the lung of a heart/lung recipient (not presented here) was also observed 


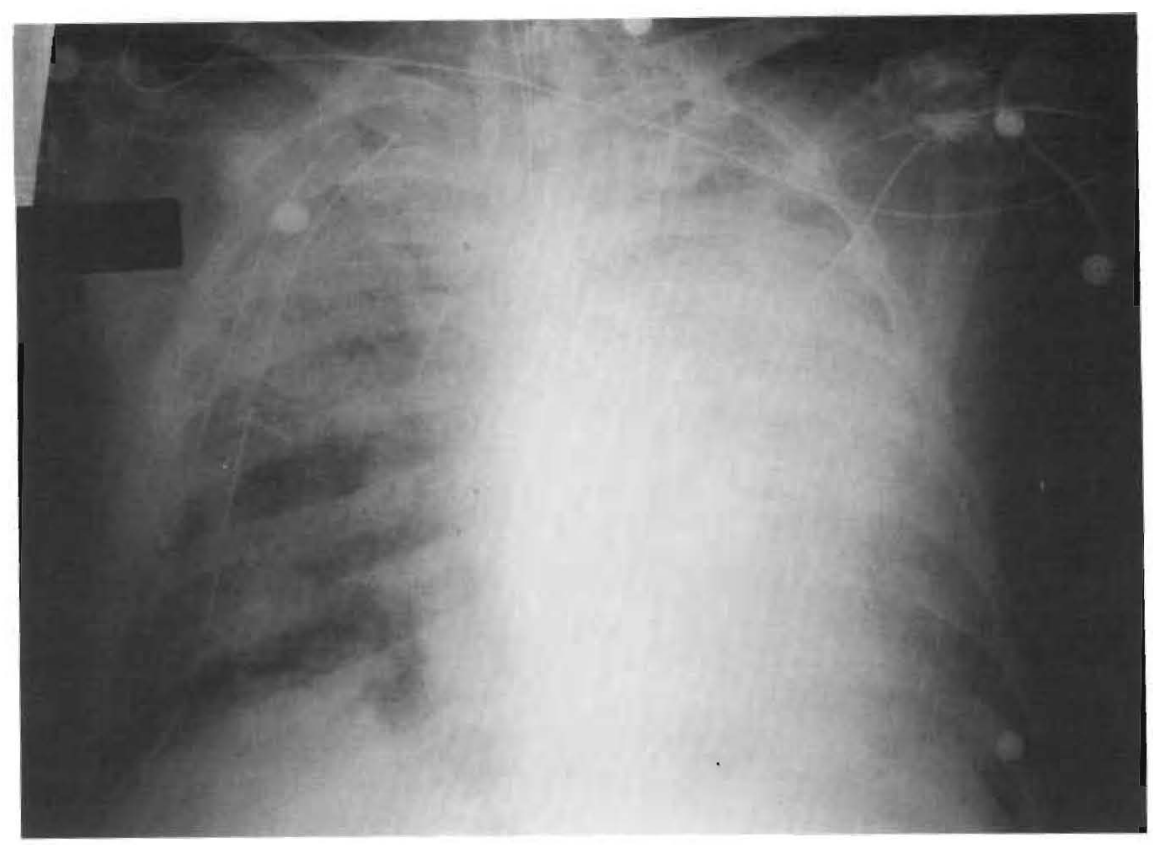

FIG 12.

Chest $x$-ray of case 5, showing severe white-out of the lungs due to Pneumocystis, CMV, and PTLD.

by us. This case, which contained a monoclonal component by immunogenotypic analysis, also regressed under the influence of reduced immunosuppression. Some time later, however, it recurred at the site of the tracheal anastomosis. Again, reduction of immunosuppression resulted in a complete remission, which continues to date.

The presence of a PTLD in the face of multiple pulmonary infections, seen in case 5, is not uncommon and suggests that some degree of association may exist between these conditions. On the other hand, they may both simply be the result of a functionally overimmunosuppressed state.

Case 6.-A 4-year-old white male underwent orthotopic liver transplantation on January 14, 1984, for treatment of biliary atresia (Fig 15). Immunosuppression consisted of CsA and prednisone. On February 5, 1986, the patient presented with 2 days' symptoms of appendicitis. At laparotomy a distal small bowel obstruction due to tumor was discovered. This was resected with a primary ileocolic anastomosis. A pathologic diagnosis of monoclonal IgM lambda PTLD was rendered and the patient was taken off all immunosuppressive medications for 6 days. Thereafter he was restarted on CsA $2.5 \mathrm{mg} / \mathrm{kg} /$ day and prednisone, $5 \mathrm{mg}$ four times a day. Postoperative abdominal computed tomography (CT) scan revealed small periaortic lymphadenopathy.

Curr Probl Surg, June 1988 


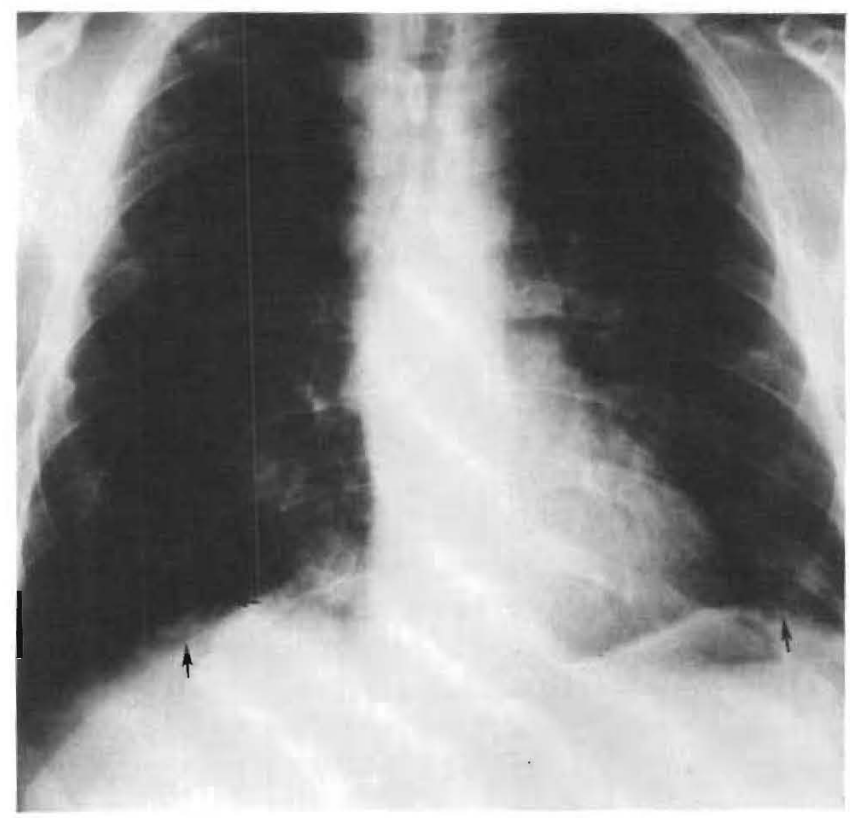

FIG 13.

Case 5. Continuing multinodular infiltrates in this patient led to open lung biopsy and diagnosis of PTLD. (From Makowka L, Nalesnik MA, Stieber A, et al: Control of posttransplant lymphoproliferative disorders and Kaposi's sarcoma by modulation of immunosuppression, in Good RA, Lindenlaub E (eds): Symposia Medica Hoechst: The Nature, Cellular, and Biochemical Basis and Management of Immunodeficiencies. Stutigart-New York, FK Schattauer Verlag, 1987, pp 567-624. Reproduced with permission.)

In May he presented with an epigastric mass, and an abdominal CT scan showed a midabdominal tumor mass $8 \mathrm{~cm}$ in diameter (Figs 16 and 17). CsA was discontinued at this time, but prednisone was continued at $5 \mathrm{mg}$ four times a day. A repeat scan in June showed no change in the size of the mass. Upper GI series with small bowel follow-through was consistent with an extraintestinal mass. A CT-directed percutaneous need]e biopsy again revealed monoclonal IgM lambda PTLD. Bone marrow biopsy was negative for tumor. All immunosuppression was discontinued in July 1986.

On September 2, 1986, the patient was readmitted. Computed tomography scan revealed multiple abdominal mesenteric masses with no abnormalities in the chest or neck. Debulking of omental tumor and biopsy of allograft liver were performed on September 11. A pathologic diagnosis of IgM lambda monoclonal malignant lymphoma was rendered. Cellular rejection with no evidence of tumor infiltration was found in the liver biopsy. The tumor again recurred and required debulking by February 1987. The histologic appearance was similar to that of previous resections. In July 1987 tumor had again recurred. It was decided to utilize antilymphoma chemotherapy to eradicate the lesion. To date, the patient has remained free of tumor and has not had resumption of chemotherapy. A more de- 


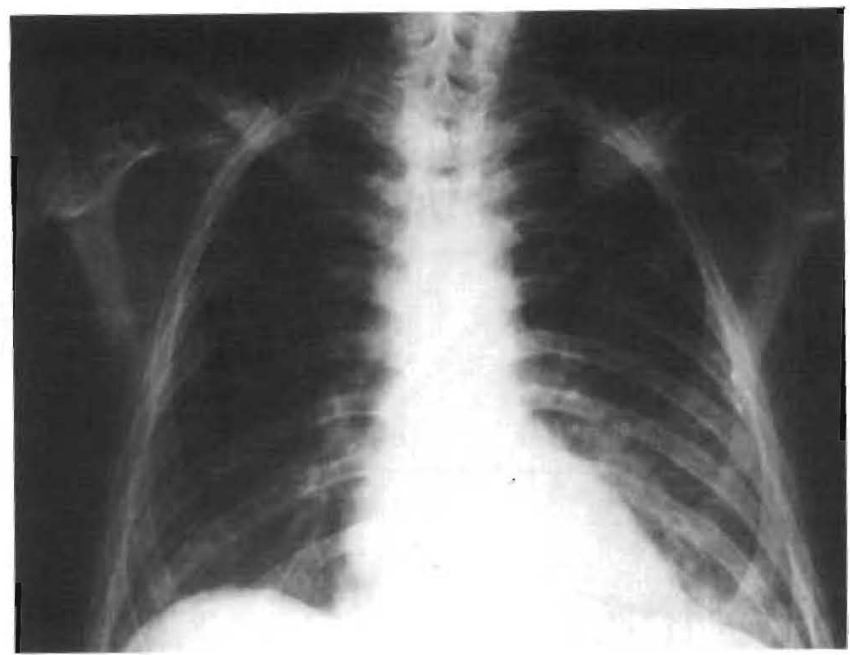

FIG 14.

Case 5. Chest $x$-ray prior to discharge showing complete resolution of PTLD. (From Makowka L. Nalesnik MA, Stieber A, et al: Control of post-transplant lymphoproliferative disorders and Kaposi's sarcoma by modulation of immunosuppression, in Good RA, Lindenlaub E (eds): Symposia Medica Hoechst: The Nature, Cellular, and Biochemical Basis and Management of Immunodeficiencies. Stuttgart-New York, FK Schattauer Verlag, 1987, pp 567-624. Reproduced with permission.)

tailed case report of this patient, who also had episodes of hypercalcemia in relation to the tumor, is underway (Jaffe $\mathrm{R}$, Nakazato et al., unpublished report).

Comment.-In this instance, the tumor did not respond to multiple attempts to induce regression by means of reducing immunosuppression. A short trial of acyclovir was also ineffective in this regard (Nakazato, personal communication, 1987). That the tumor did indeed represent multiple recurrences and not new primary lesions was proven by the identical immunophenotype of each lesion and by the identical immunoglobulin gene rearrangement pattern demonstrated by Southern blotting of the separate tumors (Locker, personal communication, 1987).

On further analysis, the tumor showed a translocation involving the long arms of chromosomes 8 and 14 in association with myconcogene translocation (Locker, personal communication, 1987). This may represent a more advanced stage of tumor progression, in which the clonal proliferation has more completely liberated itself from dependence on host factors for growth. The c-myc translocation may or may not be related to the ability of the tumor to escape immune control mechanisms. The tumor also demonstrated reduced Class II MHC antigen expression by flow cytometry. ${ }^{165}$ Defec- 


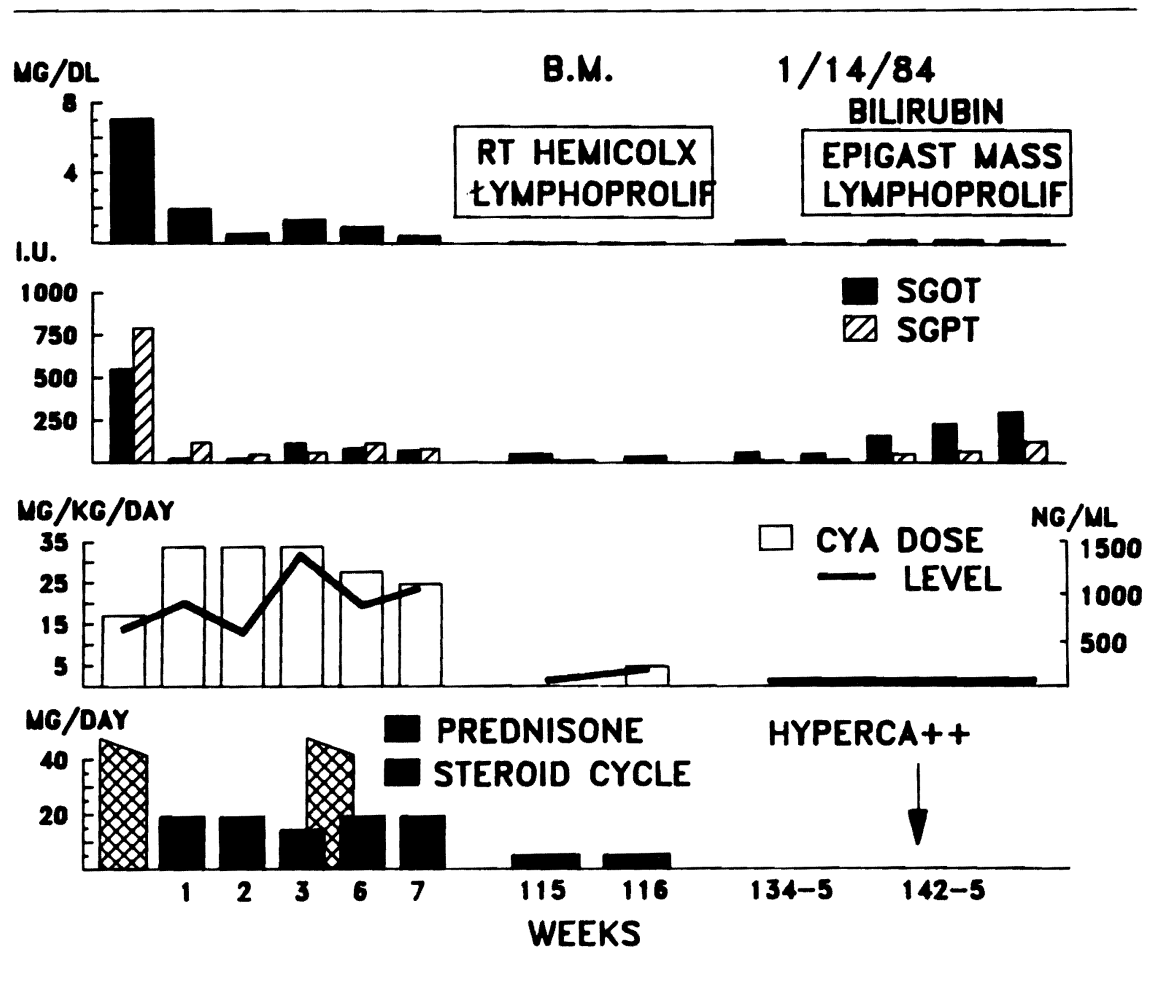

FIG 15.

Case 6. Clinical course of a 4-year-old male liver transplant recipient who presented with PTLD of the terminal ileum and sustained multiple recurrences. (From Makowka L, Nalesnik MA, Stieber A, et al: Control of post-transplant lymphoproliferative disorders and Kaposi's sarcoma by modulation of immunosuppression, in Good RA, Lindenlaub $E$ (eds): Symposia Medica Hoechst: The Nature, Cellular, and Biochemical Basis and Management of Immunodeficiencies. Stuttgart-New York, FK Schattauer Verlag, 1987, pp 567624. Reproduced with permission.)

tive (Class I) MHC expression has been noted in some cases of garden variety B-cell lymphoma ${ }^{175}$ and might conceivably contribute to reduced B-cell interaction with regulatory T-cells. We currently have no hard proof for this speculation.

In any event, it is apparent that a simple reduction of immunosuppression was not successful in this case even when combined with surgical resections, albeit incomplete. It is therefore prudent to monitor patients for signs of persistent tumor recurrence or progression throughout therapy. With this approach one will observe responses in the majority of patients who have nonclonal or regressible monoclonal lesions. These immunosuppressed individuals will thus be spared the burden of chemotherapy or radiotherapy. The few patients who do not respond to this regimen will at least have 


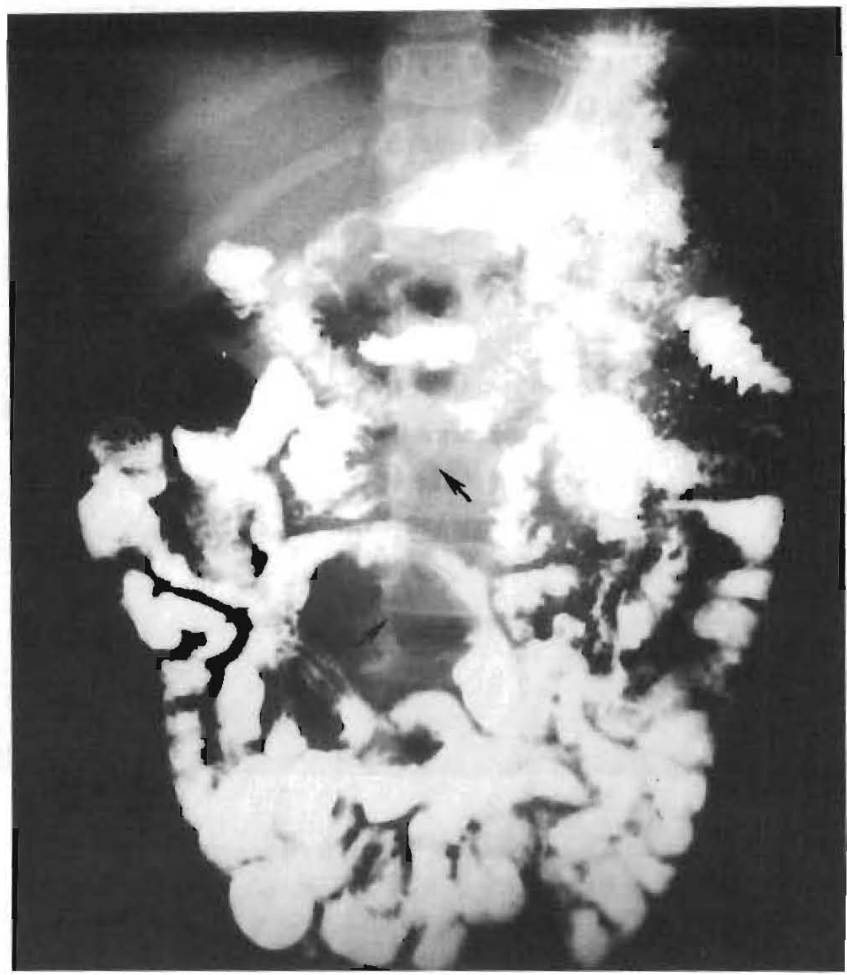

FIG 16.

Case 6. Small bowel study to investigate the first appearance of recurrent PTLD in the epigastrium. The small bowel is displaced by a large anterior epigastric mass (arrows), but no invasion of the small bowel mucosa is seen. (From Makowka L, Nalesnik MA, Stieber A, et al: Control of post-transplant lymphoproliferative disorders and Kaposi's sarcoma by modulation of immunosuppression, in Good RA, Lindenlaub E (eds): Symposia Medica Hoechst: The Nature, Cellular, and Biochemical Basis and Management of Immunodeficiencies. Stuttgart-New York, FK Schattauer Verlag, 1987, pp 567-624. Reproduced with permission.)

had a chance to partially reconstitute their immune defenses prior to undergoing more aggressive antitumor therapies.

In addition to case 3 , the following two cases will serve to emphasize the tragic consequences of delayed recognition and therapy of PTLD:

Case 7.-This 15-year-old female underwent an orthotopic liver transplant on February 20, 1972, for chronic aggressive hepatitis. Following the operation, the patient did well on prednisone and Imuran.

In April 1985 she developed fever and sore throat and became jaundiced. No specific therapy was given, and elevated liver enzyme levels apparently dropped spontaneously. She was readmitted on May 23, again for fever and sore throat. She was thought to have rejection on the basis of a liver biopsy 


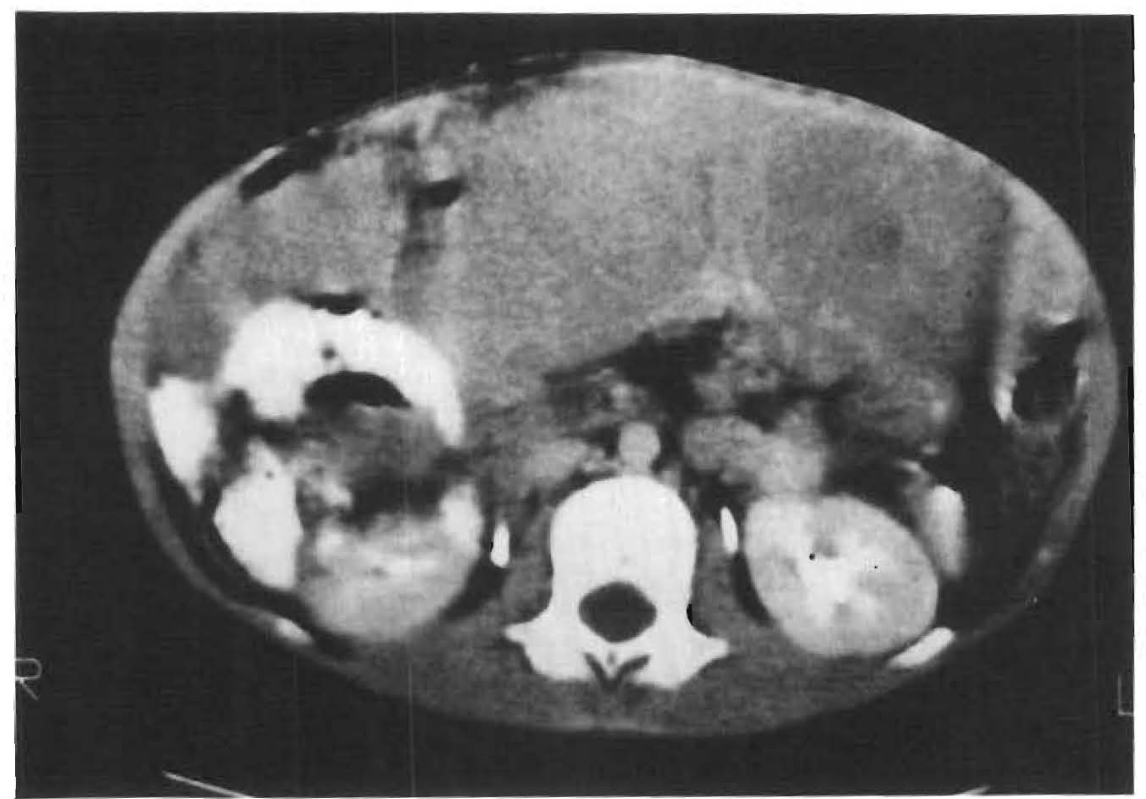

\section{FIG 17.}

Case 6. CT appearance of the epigastric mass depicted in Figure 21, further defining a large, solid, noninvasive mass anteriorly in the epigastrium. CT studies proved to be an excellent method of following this lesion. (From Makowka L, Nalesnik MA, Stieber A, et al: Control of post-transplant lymphoproliferative disorders and Kaposi's sarcoma by modulation of immunosuppression, in Good RA, Lindenlaub E (eds): Symposia Medica Hoechst The Nature, Cellular, and Biochemical Basis and Management of Immunodeficiencies Stuttgart-New York, FK Schattauer Verlag, 1987, pp 567-624. Reproduced with permission.)

and was given a steroid bolus. CsA, $200 \mathrm{mg}$ twice a day, was added to Imuran, $50 \mathrm{mg}$ four times daily, and prednisone, $10 \mathrm{mg}$ four times daily. She later developed adenopathy and in early June immunosuppression was tapered. A tonsillectomy done in the presence of coagulopathy led to acute respiratory failure and death on June 12, 1985. At autopsy, polymorphous and polyclonal PTLD was widely disseminated, involving the tonsils, lymph nodes, entire gut, thymus, allograft liver, kidneys, pancreas, salivary glands, lungs, adrenals, ovaries, and central and peripheral nervous systems.

Comment.-In retrospect, the patient's disorder began at least as early as April, in association with a serologically diagnosed primary EBV infection. Immunosuppressive medication was continued and increased even more for treatment of presumed rejection. This allowed the continued proliferation of the PTLD. Tapering of immunosuppression was begun only during the last week of the patient's life.

It may be argued that the direct cause of death was a technical complication of tonsillectomy. However, the massive tumor load 
seen at autopsy is strong evidence that the patient was in a marantic condition for which continued therapy of any type was doomed to failure.

The tumors in this case were nonclonal, indicating that technically "benign" populations of B-lymphocytes were proliferating uncontrollably due to lack of host inhibitory mechanisms. This hypothesis fits in well with the clinical course, in which the patient's immune system was effectively handcuffed by increases in antirejection medications. Several outcomes are possible in this setting: (1) uncontrolled proliferation of cells will occur, as in this case. There appears to be a "point of no return" beyond which the sheer overwhelming mass of this proliferative process overwhelms all host defenses and leads to death from any of a variety of immediate causes. (2) Proliferation of B-cells results in a genetic "accident" which causes the emergence of a single malignant clone. This clone quickly overgrows all others, leading to the death of the patient from monoclonal disease. (3) The multifactorial attack on the host's immune system favors the growth of opportunistic organisms, and the patient dies from a combination of tumor and infection.

It is apparent that these unfortunate conditions can only be prevented by an adequate index of suspicion and early diagnostic and therapeutic intervention.

In passing, it should be noted that the main immunosuppressive agent used in this case was Imuran, not cyclosporine. Two patients in our series who developed PTLDs received Imuran as the main immunosuppressive agent. Tumors in these patients arose at 68 and 163 months after transplant. In CsA-immunosuppressed patients, the mean time to onset is less than 6 months.

Case 8.-This 8-year-old female received an orthotopic liver transplant on November 1, 1982, for treatment of a hepatic inflammatory pseudotumor. She developed infectious mononucleosis in association with primary EBV infection in July 1984. Over the next 6 months she had problems with recurrent upper airway obstruction and in December 1984 underwent tonsillectomy and adenoidectomy for relief of this condition. Histologic changes were consistent with PTLD. The lesion was polymorphic and nonclonal.

Over the next 6 months there was concern because of poor linear growth; however no cause for this could be found. In October 1985 her CsA was reduced from $250 \mathrm{mg}$ four times daily to $200 \mathrm{mg}$ four times daily because of azotemia. From October through December she had been seen on several occasions by her local pediatrician because of recurrent unexplained fevers. These were diagnosed as viral illnesses and spontaneously defervesced after a few days. Immunosuppression dosages remained unchanged.

On December 17, 1985, she was admitted to Children's Hospital of Pittsburgh because of renal failure. At this time an enlarged left axillary lymph node was found. A bone marrow examination showed erythrophagocytosis consistent with viral infection.

PTLD was suspected by the transplant surgeons, and CsA was reduced 
by $50 \%$. Acyclovir was also administered. On December 27, her CsA was increased to its previous levels because of suspected liver rejection. Dosage remained at this level until January 4, when it was discontinued. A lymph node biopsy revealed a monomorphous PTLD. The patient's condition rapidly deteriorated, and she developed pericardial tamponade. Tissue from a pericardial window revealed a similar infiltrate of monomorphous PTLD. Tumor growth progressed clinically, and on January 24 chemotherapy was begun. Six days later the patient developed ecthyma gangrenosum, and biopsy showed tumor infiltration in association with aspergilli. The patient expired the following day.

At autopsy, invasive aspergillosis due to A. flavus was found to involve the brain, heart, lungs, kidneys, intestines, and urinary bladder. Obstructive thrombi due to fungus were also found in the pulmonary arteries and coronary vessels. Monomorphous PTLD was found to involve the allograft liver, kidneys, lymph nodes, bone marrow, heart, entire gut, pancreas, ovary, pituitary, thymus, parathyroid, lungs, adrenals, submaxillary gland, skin, and dura.

Comment.-This case bears many si larities to the previous case. In this instance, there was a polyme shic PTLD that preceded a more aggressive, malignant lesion by 2 years. During this interval there was clinical evidence of continuing infection, manifest first by adenoid/tonsillar hypertrophy and later by recurrent viral syndromes. It is most likely that a smoldering, low-grade proliferation of B-lymphocytes continued during this time. The time sequence, although unusual in our series, fits very well with that described by Hanto et al ${ }^{107}$ for similar lesions in non-CsA-immunosuppressed patients. They observed that early cases resemble infectious mononucleosis and later cases resemble solid tumors. In contrast, this difference is not seen in the majority of our patients, most of whom present early.

It is likely that an undefined host defect in this patient resulted in an inability to handle the virus appropriately. Therefore, the recurrent viral syndromes reflected an incubation period during which a monoclonal lesion could emerge. The relationship of the emergence of this tumor to the reintroduction of full-dose CsA during the final hospitalization cannot be defined with certainty. However, it most certainly favored tumor growth over host response, and in that sense accelerated the course of the disease. The use of chemotherapy resulted in severe invasive aspergillosis, which, coupled with tumor, was the immediate cause of death.

Since the presumed constitutional host defect in this patient is undefined, it is possible that the eventual emergence of a clonal tumor could not have been prevented. To date, this phenomenon has not been observed in any other patient in our series. With our present knowledge, however, it is still likely that earlier diagnosis, coupled with a therapeutic trial of reduced immunosuppression and, if necessary, earlier chemotherapy, could have eradicated tumor in 
this patient. The impression that early diagnosis is crucial for successful treatment of PTLD has also been expressed by others. ${ }^{259}$

\section{CASE REPORT OF KAPOSI'S SARCOMA IN A RENAL TRANSPLANT RECIPIENT}

\section{INTRODUCTION}

Kaposi's sarcoma (KS) is rare tumor normally seen in limited geographic areas. ${ }^{74 a}$ The etiology of this tumor is unknown; a causative role for cytomegalovirus is far from proven. It may present as purplish, raised, firm and nontender skin lesions. Alternatively, it may present in a more widespread form involving lymph nodes, gastrointestinal tract, or lungs. Whereas skin lesions are generally asymptomatic, gastrointestinal involvement may cause hemorrhage, pain, nausea and vomiting, or other symptoms. The diagnosis is made by biopsy, which classically shows a spindle cell neoplasm with slit-like spaces containing numerous erythrocytes. Small lesions can be resected or treated by local radiotherapy, whereas several chemotherapeutic regimens are available for more disseminated forms of the disease.

The increase of KS in AIDS patients is well known. ${ }^{43}$ Similar increases of KS have been seen in immunosuppressed transplant recipients. The frequency of this lesion in the transplant population is much less than that of PTLD. Nevertheless, it comprises a total of about $8 \%$ of tumors in transplant recipients immunosuppressed with CsA-containing regimens, compared to $3 \%$ of all tumors in conventionally immunosuppressed patients. ${ }^{185,193}$ In our series, KS is seen at an overall frequency of only $0.1 \% .{ }^{165}$ Additionally, the cases in our series have all occurred in Saudi Arabian males.

The regression of KS in transplant recipients following reduction or discontinuation of immunosuppression has been previously reported. ${ }^{190}$ For reasons that are not clear to us, this phenomenon appears to have met with much more acceptance in the medical community than has the similar behavior of PTLD. Perhaps this is so because there is some question as to whether or not KS even is a neoplasm. An intriguing proposal suggests that it may simply represent a hyperplastic proliferation. ${ }^{27 a}$

On the basis of our experience with PTLD, we suggest that a similar spectrum of tumor progression may also exist for KS. Because of the limited number of cases, lack of molecular probes for clonality, and lack of evidence for a histologic progression similar to PTLD, all cases are subsumed under the heading of KS. Based on our experience with PTLD, we would expect that the majority of cases of KS would resolve with judicious management and the reduction of immunosuppression. The possibility exists that a malignant counter- 
part of this lesion may develop in transplant patients, if sufficient mutations occur in the proliferating cells. The full spectrum of these disorders remains to be delineated.

\section{CASE REPORT}

This 35-year-old Saudi Arabian male received a cadaver kidney transplant for treatment of end-stage renal disease. Posttransplant complications included schistosomiasis of the bladder and epididymitis, both of which responded to therapy. Six months following transplant he developed purplish, raised skin lesions around the transplant scar (Fig 18). These spread to the upper trunk and arms. The diagnosis of KS was made on skin biopsy. He developed severe gastrointestinal symptoms and on radiologic examination was found to have diffuse involvement of the GI tract (Figs 19 and 20). Endoscopic biopsy of the stomach confirmed the presence of KS. ${ }^{165}$

Immunosuppressive therapy was discontinued, leading to a rapid resolution of the lesions, especially in the gastrointestinal tract. An attempt to restart low dose immunosuppressive therapy led to a rapid reappearance of skin and gastrointestinal lesions. Thus, CsA and prednisone were again discontinued, resulting in a resolution of the recurrent lesions.

Although the patient has retained his kidney for approximately 1 year,

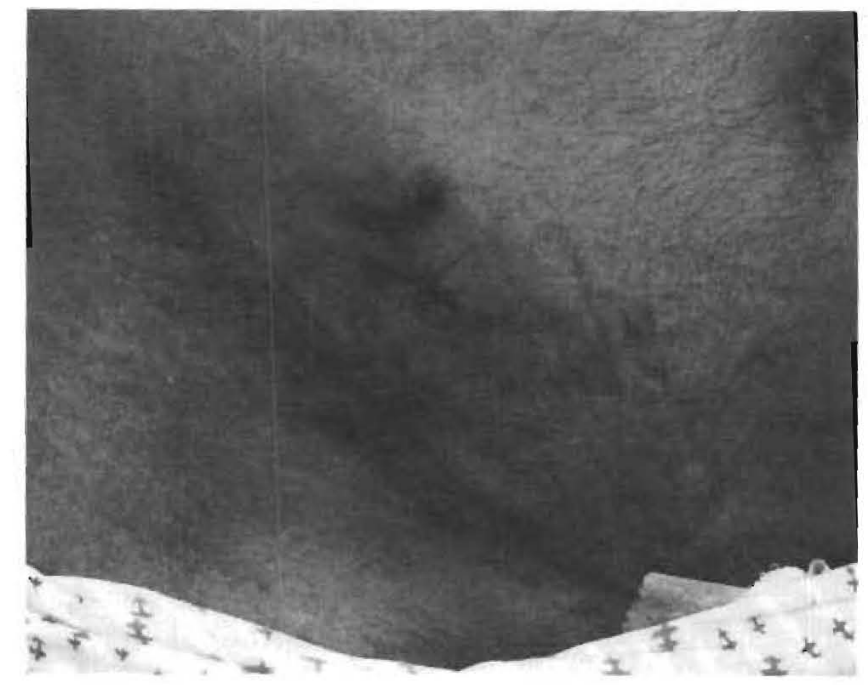

\section{FIG 18.}

Kaposi's sarcoma in a renal transplant recipient. The skin lesions of Kaposi's sarcoma can be seen in conjunction with the transplant scar. (From Makowka L, Nalesnik MA, Stieber A, et al: Control of post-transplant lymphoproliferative disorders and Kaposi's sarcoma by modulation of immunosuppression, in Good RA, Lindenlaub E (eds): Symposia Medica Hoechst: The Nature, Cellular, and Biochemical Basis and Management of Immunodeficiencies. Stuttgart-New York, FK Schaltauer Verlag, 1987, pp 567-624. Reproduced with permission.) 


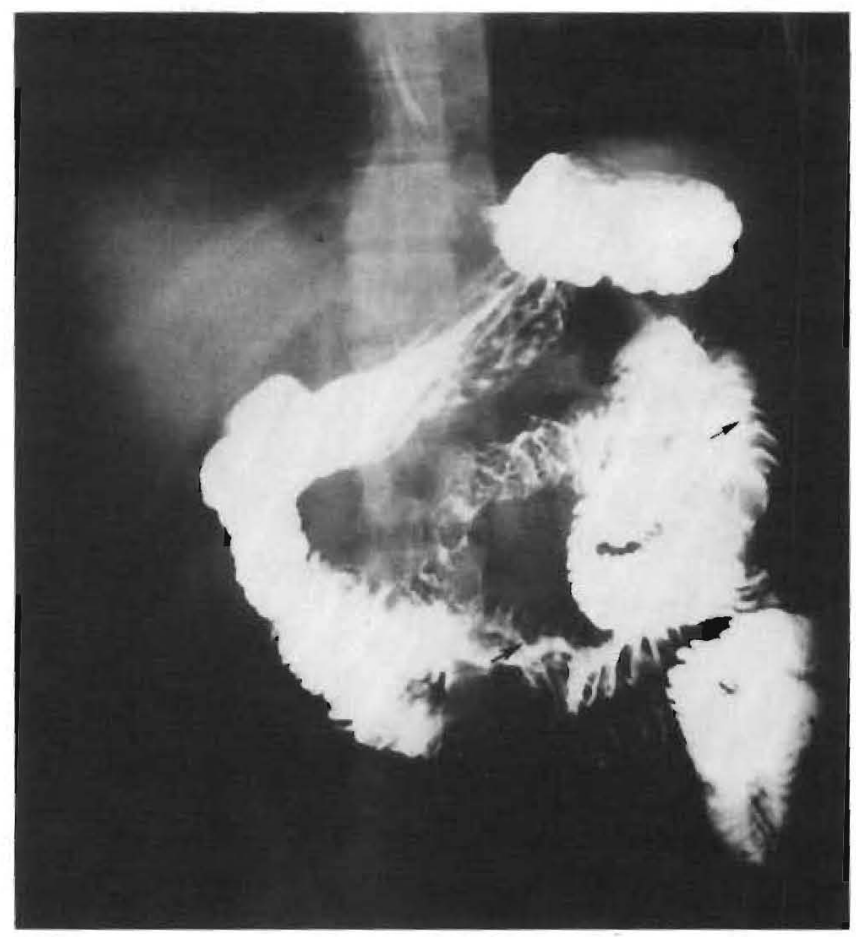

FIG 19.

Small bowel series demonstrating diffuse widespread involvement of Gl tract with biopsyproven Kaposi's sarcoma. Arrows demonstrate thickened mucosal folds due to tumor. (From Makowka L, Nalesnik MA, Stieber A, et al: Control of post-transplant lymphoproliferative disorders and Kaposi's sarcoma by modulation of immunosuppression, in Good RA, Lindenlaub E (eds): Symposia Medica Hoechst: The Nature, Cellular, and Biochemical Basis and Management of Immunodeficiencies. Stuttgart-New York, FK Schattauer Verlag, 1987, pp 567-624. Reproduced with permission.)

renal function studies indicate slow and progressive rejection. He remains well with no evidence of gastrointestinal lesions but with minimal skin lesions (Fig 21).

\section{COMMENT}

Again we see a dramatic response of the host-tumor unit following modulation of the immune response. Kaposi's sarcoma, which arose in this patient following a disabling of the immune response by CsA and prednisone, regressed when the cells were allowed to resume their normal functions. The persistence of minimal skin lesions indicates that the final verdict on this case is not yet in. From a clinical standpoint, however, it is obvious that the patient would have expired with tumor had not the appropriate actions been taken. There- 


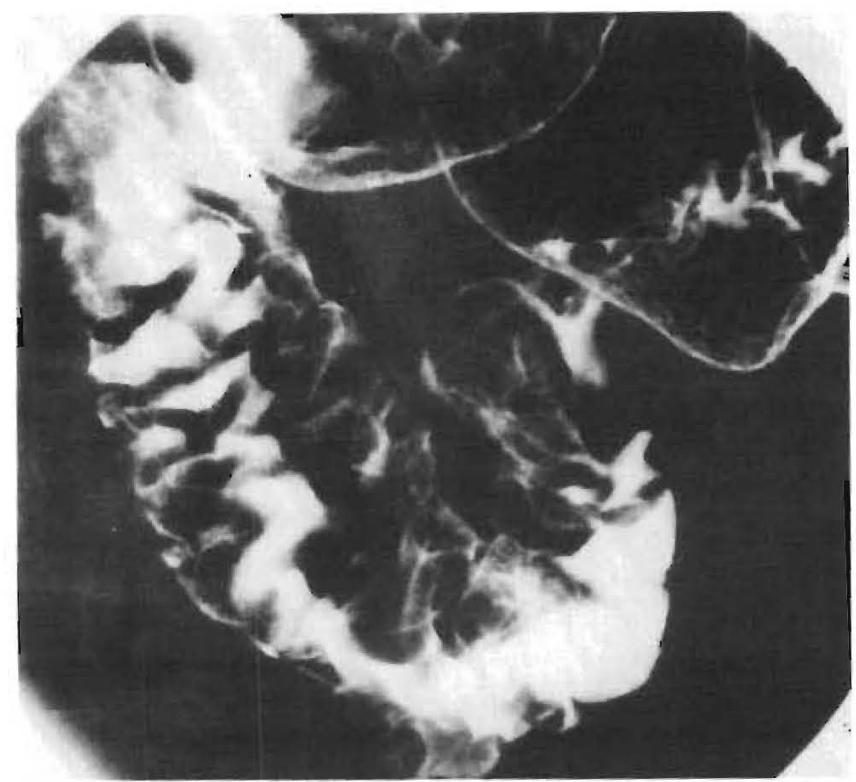

FIG 20.

Close-up of small bowel series, demonstrating prominent folds in stomach infiltrated with tumor. (From Makowka L, Nalesnik MA, Stieber A, et al: Control of post-transplant lymphoproliferative disorders and Kaposi's sarcoma by modulation of immunosuppression, in Good RA, Lindenlaub E (eds): Symposia Medica Hoechst: The Nature, Cellular, and Biochemical Basis and Management of Immunodeficiencies. Stuttgart-New York. FK Schattauer Verlag, 1987, pp 567-624. Reproduced with permission.)

fore, while the biology of this disorder is being debated, there can be no question as to the clinical approach to the treatment of KS in transplant recipients: reduction of immunosuppression and management of local complications. This case is presented to demonstrate the similarity in therapeutic approach between these two disparate tumors, PTLD and Kaposi's sarcoma, which arise under similar circumstances.

\section{CONCLUDING COMMENTS}

At the outset of this monograph it was stated that the immune system plays a key role at every step in the pathogenesis of PTLD. By analogy, the surgeon plays a key role at every step in the cure of this disease. These steps include (1) the maintenance of allograft tolerance using the minimum amount of immunosuppressive medication; (2) maintaining a high level of suspicion for PTLD in the setting of viral syndrome with lymphadenopathy, organ dysfunction etc.; (3) prompt biopsy of appropriate tissue specimens for diagnostic pur- 


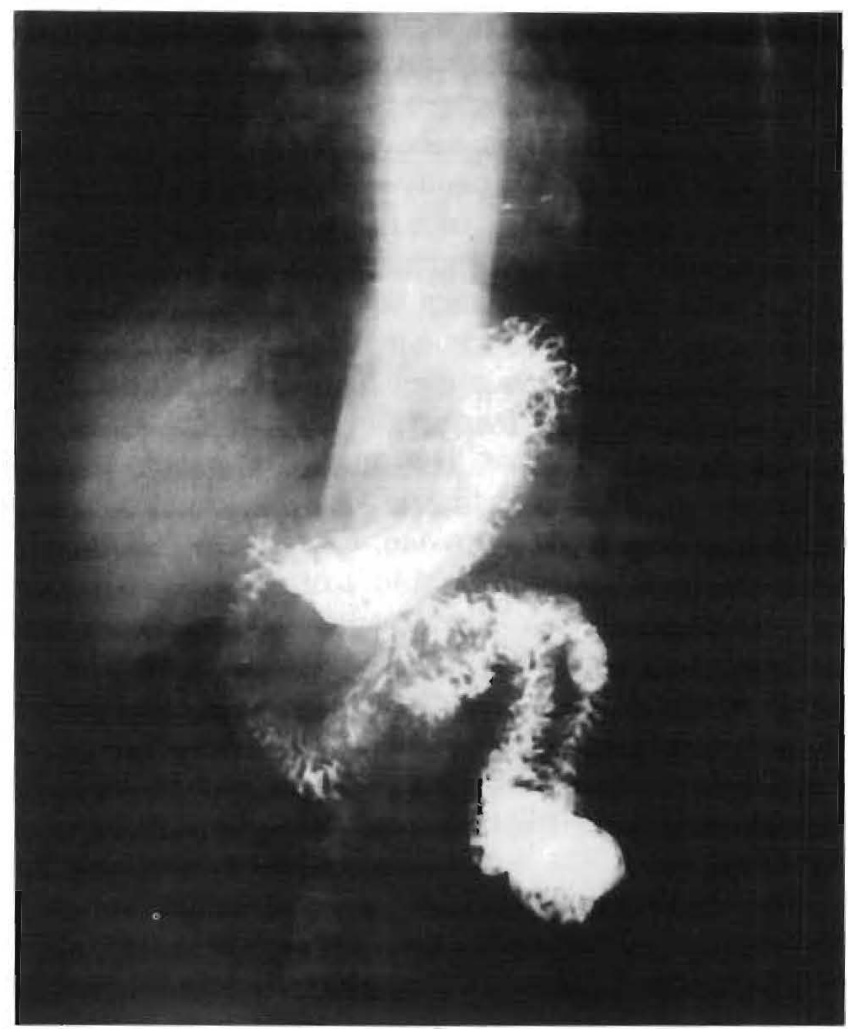

FIG 21.

Follow-up Gi series after complete discontinuation of immunosuppression and resolution of Gi symptoms demonstrates normal mucosal pattern and no evidence of KS. (From Makowka L, Nalesnik MA, Stieber A, et al: Control of post-transplant lymphoproliferative disorders and Kaposi's sarcoma by modulation of immunosuppression, in Good RA, Lindenlaub E (eds): Symposia Medica Hoechst: The Nature, Cellular, and Biochemical Basis and Management of Immunodeficiencies. Stuttgart-New York. FK Schattauer Verlag, 1987, pp 567-624. Reproduced with permission.)

poses; (4) close collaboration with the pathologist to ensure the appropriate handling of tissue for clonal analysis, karyotype, and viral analysis at a minimum; (5) willingness to challenge a pathologic diagnosis of generic non-Hodgkin's lymphoma in a transplant recipient; (6) provision of emergency surgical intervention for complications of large or strategically placed PTLDs; (7) judicious reduction of immunosuppression as a primary intervention for the control of PTLD.

The recognition that most cases of PTLDs represent treatable tumors removed the specter of hoplessness previously attached to the diagnosis of lymphoma in the transplant patient population. Similarly, a realization that the powerful immunosuppressant Cyclospor- 
ine A did not result in a large increase in the number of such tumors allowed numerous allograft recipients to enjoy the benefits of this drug, and allows the further development of immunosuppressive agents that act by similar mechanisms.

Presently, several centers are analyzing these tumors for hints that relate to the general questions of tumor progression and host immune control of tumors. Others are investigating the application of a vaccine against the Epstein-Barr virus. Early enthusiasm for the usefulness of such a vaccine in control of lymphoid tumors has recently been downplayed. However, perfection of such a vaccine would undoubtedly play an important role in removing the major known etiologic factor of these lesions and thus preventing tumor development. The human and financial savings offered by such an ability would be substantial. Likewise, dissection of the mechanism by which the immune system is able to cause regression of PTLD may give insight into forms of immune augmentation therapy suitable for some cases of AIDS lymphomas.

The dream of the transplant surgeon is to achieve a specific allograft tolerance in the recipient without compromise of any other function of the immune system. This goal will no doubt be realized. When it is, PTLDs and similarly arising tumors will no longer be seen.

Meanwhile, we should seize the opportunity provided by this experiment of nature-and man-to understand how a serious but uncommon side effect of organ transplantation actually reflects a perturbation of delicately balanced systems that have evolved over the millennia.

\section{APPENDIX: TECHNICAL CONSIDERATIONS RELATED TO THE STUDY OF POSTTRANSPLANT LYMPHOPROLIFERATIVE DISORDERS}

REVIEW OF IMMUNE INTERACTIONS AND RELEVANT METHODS OF ANALYSIS

\section{Cellular Interactions}

Manipulation of the immune response produces a tolerant state that allows survival of a foreign graft. It also provides the backdrop, in the appropriate setting, for the development of PTLD. Reconstitution of the immune response is a contributor to the rejection of PTLD, but this may also precipitate allograft rejection. A comprehensive review of transplantation immunology and tumor immunology is beyond the scope of this monograph, but it is appropriate to review some fundamental aspects of the immune response in order to provide a context from which to discuss the pathogenesis and behavior of PTLDs. 
The major cellular components of the immune system are the lymphocytes and macrophages. Lymphocytes are most simply subdivided into two general classes: B-lymphocytes, which are responsible for antibody production, and T-lymphocytes, which include effector cells and regulatory (helper and suppressor) cells. (Other cells, such as NK cells, which also play important roles in the immune response, and considered below). Following an antigenic challenge to the host, the normal immune response can also be divided into two interconnected stages, namely the induction (afferent) phase and the effector (efferent) phase (Fig 22). During the induction phase, a carefully orchestrated interaction occurs first between host immune cells and the inciting agent (antigen) and later among the various host immune cells. The outcome of this cellular conversation is the selection, activation and proliferation of that subset of cells which, under ideal circumstances, is best suited to deal with the antigenic challenge. The effector phase represents the vector sum of all actions taken by the recruited cells to eliminate the antigen. Some, but not all, of these effector mechanisms are also illustrated in Figure 22.

In parallel with the induction and effector phases, a specific regulatory network evolves in order to terminate the immune response at the appropriate time. Simultaneously, selected immune cells are diverted from the main pool to contribute to the cumulative and long-lived immunologic memory system of the host.

The actions of T-lymphocytes and B-lymphocytes are best considered separately, although they invariably act in concert during the integrated immune response. T-cells respond to antigen that has been processed by an antigen-presenting cell, usually of macrophage origin. The antigen is recognized only in association with certain molecules (major histocompatibility or MHC antigens) present on the surface of the antigen-presenting cell. The presence of specific MHC antigens provides a means by which the immune system can distinguish "self" from "non-self" cells. "Non-self" cells have either different MHC antigens, in which case they will be recognized as foreign, or no MHC antigens, in which case they will not be recognized by this mechanism. This dual recognition forms the basis of "MHC-restriction" of many T-cell mediated immune responses. ${ }^{273}$

A variety of T-cells with different functions may respond to the antigen-MHC complex. ${ }^{208}$ Cytotoxic T-lymphocytes mediate direct killing of cells or organisms containing the inciting antigen. The actions of cytotoxic T-cells are up-regulated by factors elaborated from T-helper lymphocytes and down-regulated by factors secreted from T-suppressor cells. A separate set of T-cells is involved in secreting factors that activate macrophages as part of the delayed type hypersensitivity response. These T-cells are also under the control of helper and suppressor T-subsets. In addition, T-helper and T-sup- 


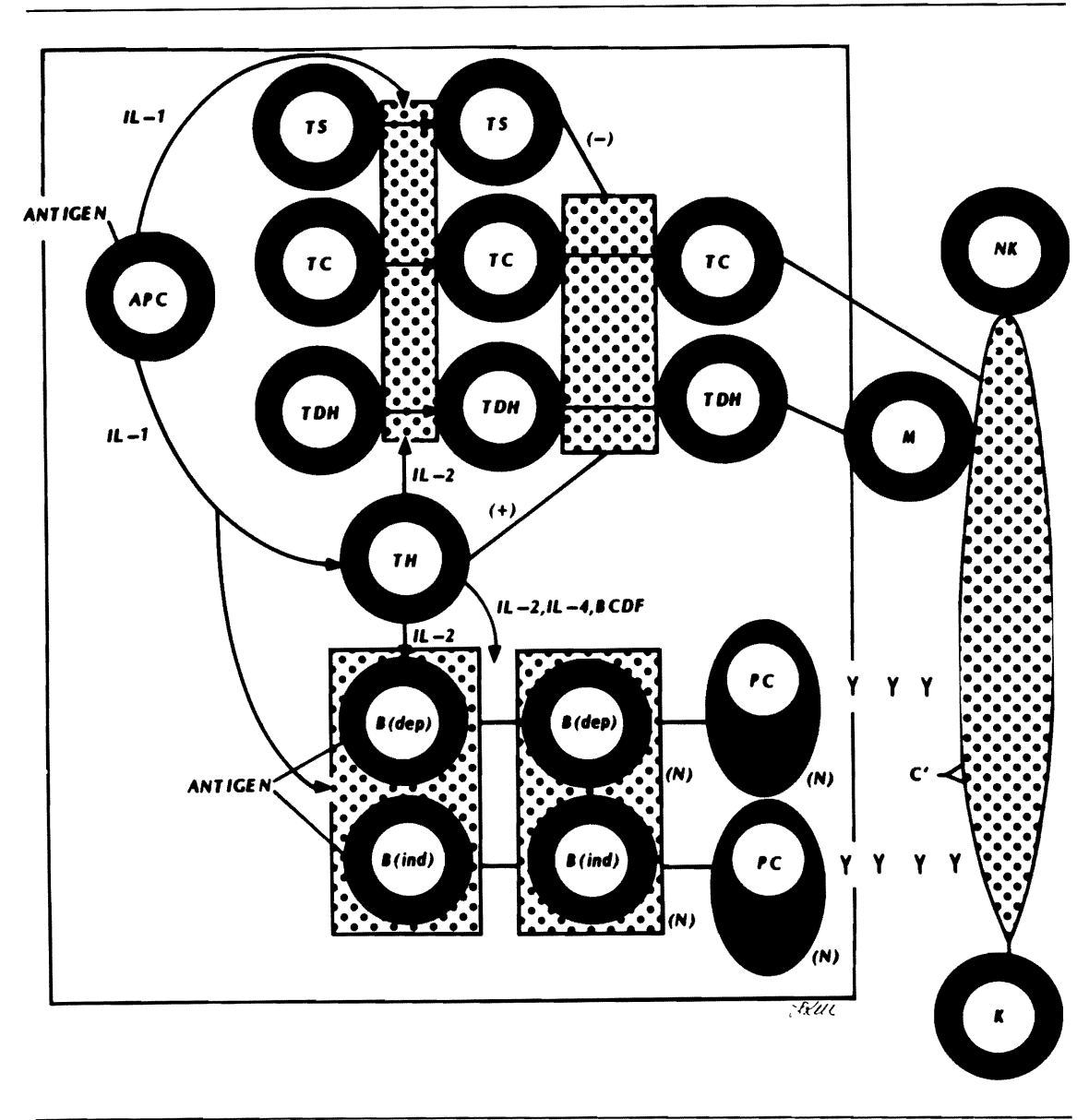

FIG 22.

Schematic representation of afferent (within box) and efferent (outside box) cellular immune interactions. Antigen combines with antigen-presenting cell (APC), usually a macrophage and interacts with a specific resting T cell (not shown). With the help of IL-2 the $T$ cell is activated into a helper cell (Th) which secretes a variety of lymphokines, notably IL-2. This lymphokine in conjunction with IL-1 activates other T cells, including suppressor (Ts), cytotoxic ( $T c)$ and delayed hypersensitivity $(T d h) T$ lymphocytes. T helper cells exert a positive influence, and $T$ suppressor cells a negative influence, on subsequent development of the T cell system into mature effector cells. Meanwhile, B cells also interact with antigen either in conjunction with (Bdep) or independent from (Bind) direct interaction with $T$ cells. $A$ variety of interleukins are required to drive subsequent $B$ cell proliferation and differentiation into antibody-producing plasma cells (PC). In the efferent arm of the immune response, a variety of products deal with antigen-bearing structures (oval). Cytotoxic $T$ cells may directly attach to and inflict damage upon the target. Delayed hypersensitivity cells marshal a number of factors to deal with the target. Some results are directly mediated by macrophages $(M)$. Natural killer cells $(N K)$ also inflict damage directly similar to cytotoxic $T$ cells. Antibodies attach directly to the target and may aggregate it for sub- 
pressor cells influence the growth of certain responding B-lymphocytes in opposing fashions.

A schema depicting the central role of the helper T-lymphocyte in the cascade of reactions necessary for an effective immune response is shown in Figure 22. In this example, a macrophage with specific surface receptors recognizes a foreign antigen and acts as the antigen presenting cell. A T-helper lymphocyte with receptors to the same antigen recognizes this antigen on the surface of the macrophage in association with self-MHC antigens. This recognition event triggers a number of intracellular responses within the T-cell, resulting in its activation. ${ }^{208}$ The activated T-cell produces a number of functional molecules, some of which remain on its cell membrane as receptors, and others which are secreted into the extracellular space to act on nearby cells. The secreted substances are called lymphokines.

Lymphokines, which were originally thought only to affect cells of the immune system, are now known to have effects as diverse as hepatocyte stimulation and sleep induction. For these reasons they are also referred to as cytokines. Nevertheless, their major role is to form an amplification mechanism bridging the induction and effector phases of the immune response. ${ }^{73}$

Interleukin-1 is produced by monocytes and is more properly considered a monokine. This molecule acts as a cofactor in the activation of T-cells, facilitates the production of other lymphocytes and also acts as a cofactor in B-cell activation. The activated T-cell produces interleukins 2, 3 and 4, gamma interferon and B-cell differentiating factor ${ }^{73}$ Since the activated T-cell also expresses surface receptors for interleukin-2, a positive feedback loop is established. Interleukin-2 causes clonal expansion of the activated $T$ cells, an effect that also requires the continued presence of antigen.

A positive feedback loop is also established by the production of gamma interferon. This lymphokine, among its many other effects, increases MHC expression on antigen presenting cells. This facilitates antigen presentation and thus activation of T-cells. ${ }^{73}$

$\mathrm{B}$-cell responses to antigen can be categorized as either T-dependent or T-independent. A T-dependent response is defined as one that requires two steps for B-cell activation: (1) recognition of a foreign antigen by a B-lymphocyte with specific receptors, and (2) recognition of the antigen-MHC complex on the surface of the B cell by a helper T-lymphocyte. In contrast, a T-independent response requires only the initial interaction between B-lymphocyte and antigen

sequent removal. Alternatively they may serve as a bridge for activated complement, resulting in "holes" in the target. Antibody may also serve as a bridge for the attachment of killer lymphocytes $(K)$. This is referred to as antibody-dependent cellular cytotoxicity $(A D C C)$. Not shown are immune dampening mechanisms and memory cell generation. 
to occur in order to trigger B-cell activation. In either case, further proliferation and differentiation of the activated $B$ cell is dependent upon the presence of lymphokines. ${ }^{126,139}$ The activated B-lymphocyte is acted upon by interleukin-4 (B-cell stimulating factor 1) and B-cell differentiating factor (B-cell stimulating factor-2). These lymphokines may act by stimulating a variety of B-cell surface receptors in a sequential pattern. ${ }^{44,139}$ Other lymphokines serve to enhance or diminish the effects of these molecules. The end result is B-cell proliferation and differentiation into antibody-producing plasma cells. Thus, in a strict sense, all B-cell responses have dependence on $\mathrm{T}$ lymphocytes. This pathway is outlined in Figure 22 and a representation of the antibody molecule is shown in Figure 23.

Despite the exquisite interdependence of the various components of the immune system, the redundancy of these interconnections must be stressed. If one pathway of response is blocked, several others are available to take its place. This redundancy is also manifest as a multiplicity of mechanisms available to perform a single function. The effector function of immune cytotoxicity is a case in point.

A cell bearing an antigenic stimulus may be lysed by one of several mechanisms (see Fig 22). An antibody molecule attached to the cell surface may activate the complement system, leading to the formation of a membrane attack complex. This complex forms small tubules that span the full thickness of the cell membrane leading to cell lysis. Alternatively, the antibody molecule may serve as a bridge to connect the target cell to an effector lymphocyte known as a $\mathrm{K}$ (killer) cell. The reaction mediated by this cell is known as antibodydependent cellular cytotoxicity (ADCC). NK cells (natural killer cells), which may be important in tumor resistance, effect target cell lysis by mechanisms similar to that used by the complement system. These cells attach directly via specific NK determinants on the cells. The relationship of $K$ cells and NK cells to other lymphocytes is unresolved at present. Indeed, it is not known whether these activities are mediated by the same cell type. Therefore it is best to consider "K cell" and "NK cell" as functional definitions of the cells mediating ADCC and NK activities, respectively.

The cytotoxic T-lymphocyte represents yet another entity capable of lysing a target cell. As is the general case for T-cell recognition of antigen, the cytotoxic reaction in this case is limited by MHC-restriction. Macrophages may also act as immune effector cells by virtue of their phagocytic and enzymatic properties.

The interrelationship of B-cells and T-cells also forms an important link in the pathogenesis of PTLD, especially in terms of control mechanisms. In this regard, E. Klein has emphasized that B-cell proliferation is normally under tight T-cell control. She suggests that these normal physiologic immune control mechanisms may also be operative in control of rampant EBV-associated B cell lymphoproli- 


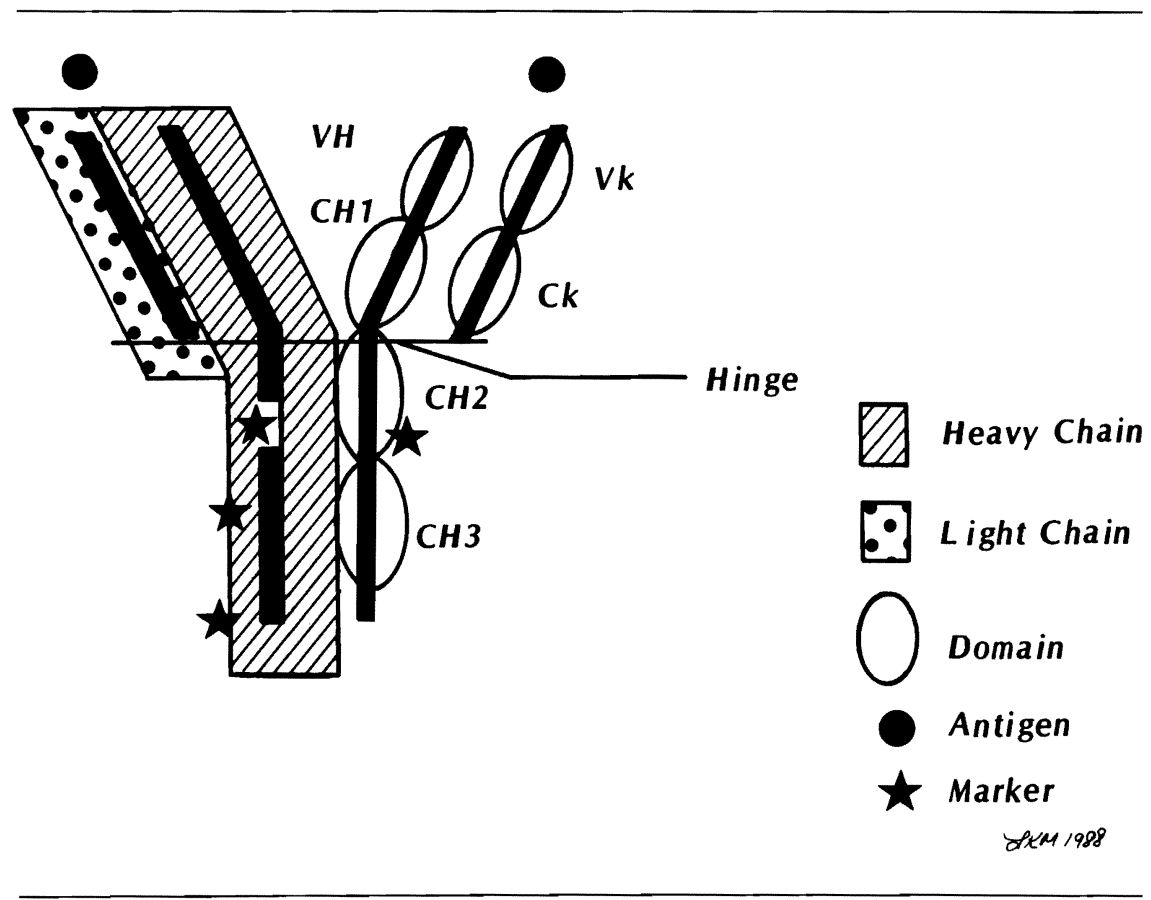

FIG 23.

Schematic representation of antibody molecule. This IgG molecule is composed of two heavy chains and two light chains. On the left side of the picture, heavy and light chains are contained withiin separate boxes. On the right side, they are broken down into defined globular regions called domains. The light chain, in this case a kappa chain, is composed of one variable $(V k)$ and one constant (CK) domain. The heavy chain, which in the case of IgG is called a gamma chain, is composed of one variable $(\mathrm{VH})$ and three constant $(\mathrm{CH} 1$, $\mathrm{CH} 2, \mathrm{CH} 3$ ) chains. There is some degree of mobility at the hinge region, where the chains are held together by disulfide bonds (not shown). Antigens are bound by the variable regions of heavy and light chains, as illustrated. In contrast, most of the tags used to visualize antibodies in various immunocytochemical techniques (e.g., fluorescent, enzyme, or radioactive tags) bind to the heavy chains at sites removed from the antigen attachment. These areas are normally responsible for other activities, e.g., complement binding.

feration. ${ }^{141}$ Homeostatic T-B control mechanisms have also been suggested to play a role in the down-regulation of B-cell proliferation in uncomplicated infectious mononucleosis. ${ }^{140}$ However, as shown in the previous discussion, current models of PTLD stress the cytotoxic T-cell response as the predominant host control mechanism in this disorder.

\section{Methodologies Employed in the Identification of Lymphocytes}

The cooperative interactions between T- and B-lymphocytes were totally unknown prior to the $1960 \mathrm{~s}$. This was due to an inability to 
distinguish these cell types, which have similar appearances at a microsopic and ultrastructural level.

Clearly, a method for separating these cells was needed. The solution was to be found in the presence of specific molecules, located on or within the cells, that could be utilized as specific markers for individual cell subpopulations. The methods that were developed also provided a means by which the clonal composition of lymphoproliferative disorders, including PTLDs, could be evaluated.

The technique used to locate such molecules had originally been devised by Coons in 1942 to identify Pneumococcus within tissue sections. ${ }^{52 a}$ Known as the fluorescent antibody technique, it exploited the exquisite sensitivity of the antigen-antibody reaction to locate specific molecules of interest. In theory, the concept was simple: if an antibody could be raised against a particular antigen, then it would react with that antigen. Further, if the antibody could itself be made visible, then the reaction would by inference show all locations of the specific antigen. It was discovered that antibodies could be combined with such visualizing reagents and still maintain the ability to combine specifically with antigens. This was so because the antibody-antigen combining site and the site amenable to combination with visualizing reagents were located on two separate portions of the antibody molecule (see Fig 23). A fluorescent compound derived from the interaction of 4-amino fluorescein and the World War I poison gas phosgene served as the marker in the first set of studies and prompted the descriptive term fluorescent antibody. Since that time, a variety of compounds and modifications have been used to identify antigens, all based on the same principle. The names of the specific techniques derive from the identity of the visualizing reagent conjugated to the antibody molecule. For example, immunoperoxidase techniques utilize the enzyme horseradish peroxidase to localize the antigen-antibody reaction, immunogold techniques utilize conjugated gold particles, and so on. The terms immunocytochemical or immunohistochemical are interchangeably applied to refer to this family of techniques. The methods are most frequently used to identify antigens in tissue sections (immunohistochemistry) or in cell suspensions (immunocytochemistry). Automated tabulation of cells stained with one or more fluorescent antibodies is one basic unit of measurement in flow cytometric techniques.

The efficacy of the marker is of little use in the absence of the appropriate antibody. The discovery of antibodies specific for B- or T-lymphocytes required a number of years and the advent of monoclonal antibodies to reach fruition.

Early studies of lymphocytes took advantage of the fact that B-cells were the source of antibodies. Thus, by raising antibodies against antibodies one would be able to identify these cells eo ipso. How- 
ever, the only practical means of producing specific antibodies was by immunization of animals, collection of serum, and isolation of the gamma globulin (antibody-containing) fraction. A single antigen provokes a multitude of different B-cells to produce an entire population of antibodies that are unique but share in common some degree of reactivity to the antigen. Such a collection of antibodies is referred to as polyclonal to reflect this diverse B-cell origin. The investigator is also restricted because the immune system will preferentially respond to highly antigenic structures. Thus molecules that are present in minute quantities will evoke little or no detectable response in this model.

In 1975, Kohler and Milstein devised a means whereby the unique antibodies produced by clones derived from individual B-cells could be isolated in large quantities. ${ }^{149 a}$ This discovery of monoclonal antibodies revolutionized the field of immunology and led in 1984 to the Nobel prize for these investigators. ${ }^{263 a}$

The original technique was based on the idea of fusing the highly developed antibody production machinery of the plasma cell (mature B-cell) together with the genetic blueprints for the assembly of an antibody of desired specificity. Since the DNA instructions reside in the nucleus and antibody production occurs in the cytoplasm, a functional product from such a fusion was at least theoretically possible. Malignant plasma cells (plasmacytoma cells) were chosen to contribute the cytoplasmic component and splenic B-cells were chosen to donate the nuclear component. Cells were fused by exposure to Sendai virus and manipulated in a fashion to select the hybrid population. Individual cells were then separated and grown in vitro. The supernatants of the monoclonal populations were assayed for (monoclonal) antibody activity, and appropriate clones were propagated in vitro or in vivo.

Armed with monoclonal antibodies and immunocytochemical techniques, investigators were at last able to identify specific molecules that were limited in cellular distribution. A series of T-cell differentiation antigens were identified in 1980, leading to an explosive and decentralized number of discoveries of so-called maturation antigens on T-cells, B-cells, and other cell types.

A short period of increasing confusion resulted due to the plethora of monoclonal antibodies, each with idiosyncratic names, that were raised against lymphocytes. This situation was addressed and corrected by two international workshops held in Paris (1982) and Boston (1984) and devoted to nomenclature and standardization of such reagents. ${ }^{207}$ The result was the identification of differentiation clusters (CDs) that were used to designate antigens present on Bcells, T-cells, or both, at various times within the cell's lifetime. ${ }^{112,207}$ Lymphocyte subpopulations will be referred to in this monograph by CD number. 


\section{Methodologies Employed for the Determination of Clonality}

Immunocytochemical Techniques.-During maturation, a B-lymphocyte produces immunoglobulins. A single B-cell (and all of its progeny) will produce antibody molecules constructed of one set of light chains (kappa or lambda) and one set of heavy chains (IgM, A, D, G, or E) (see Fig 23). As the cell matures, the expression of heavy chain may change from one type to another, but the light chain remains invariant. In other words, a kappa-producing B-cell will never produce lambda light chains, and vice versa.

Since a cell produces either kappa or lambda light chain only, this provides a means by which one can estimate the clonal status of a B-cell proliferation. Normally, the ratio of kappa-bearing B-cells to lambda-bearing B-cells is $2: 1 .^{112}$ Thus, a benign, reactive polyclonal proliferation of B-lymphocytes would contain cells in this approximate ratio. Conversely, a monoclonal proliferation of B-cells is derived from a single cell. Therefore one would expect the overwhelming majority of cells in this case to express the same, i.e., either kappa or lambda, light chain.

The total expression of antibody molecules and other cell markers that can be detected in a particular tumor by these methods is referred to as the immunophenotype of the tumor. Utilizing a combination of histopathology, immunophenotypic analysis, and clinical information, at least 17 different types of non-Hodgkin's lymphoma can now be recognized. ${ }^{112}$ Despite the contribution of immunophenotypic analysis to the classification of lymphomas, some limitations remain.

During most of its life, the B-cell expresses immunoglobulin on its surface. It is not until the latter stages of maturation, especially as the cell becomes a plasma cell, that significant amounts of immunoglobulin accumulate in the cytoplasm. ${ }^{112}$ Unfortunately, routine histologic processing (i.e., formalin fixation and paraffin embedding) masks the surface immunoglobulins and typically demonstrates only cytoplasmic immunoglobulins. (For this reason it is essential that the surgeon provides the pathologist with fresh tissue when lymphoid tissue is submitted for diagnostic evaluation. Frozen sections may be prepared from this tissue for demonstration of surface, as well as cytoplasmic, markers. In addition, cell suspensions may be prepared for immunophenotypic analysis and the results quantified directly).

A separate set of problems exists in the use of immunoglobulin stains to interpret clonality due to the biology of the B-lymphocyte itself. Very primitive B-lymphocytes do not yet express immunoglobulins, thus preventing clonal analysis of primitive B-cell tumors by this technique. Such tumors have been referred to in the past as "null-cell lymphomas." ${ }^{49}$ Immunocytochemical methodology also is 
not very good at detecting the presence of a monoclonal proliferation in a polyclonal background. If the normal polyclonal kappa:lambda ratio is $2: 1$, at what point does one assign a cutoff that will define a proliferation as monoclonal? The literature is not clear on this subject. ${ }^{158,181,242}$ However, it became apparent that a more sensitive indicator for diagnostically distinguishing polyclonal from monoclonal lymphoid proliferations was desirable.

Recombinant DNA Techniques.-Simultaneously with the development of these phenotypic measures of B- and T-lymphocyte identity and maturation, a different group of investigators was making seminal discoveries in regard to the genetic maturation of lymphocytes.

Ever since the discovery of the molecular structure of DNA in 1953, investigations into the nature of the nucleic acids had been limited by the massive size and heterogeneity of these compounds. In this regard, the situation was analogous to the restrictions imposed on immunologists by polyclonal antibodies, and it required a pivotal insight into a means whereby DNA could be made available in a more defined form to investigators.

In 1970, Smith at Johns Hopkins discovered a bacterial enzyme that would cleave DNA at a specific site. ${ }^{238 a}$ This enzyme, referred to as a restriction endonuclease, was the immediate forerunner of a large family of enzymes capable of cutting DNA at unique sites. To date, over 100 restriction endonucleases have been identified, giving the molecular biologist an incredible ability to dissect out reproducible fragments of DNA. An additional advantage of most restriction endonucleases lies in the fact that the scission of DNA takes place in an asymmetric fashion, implying that the ends may recombine, or reanneal, in a specific fashion. This behavior may be exploited to produce large quantities of specific DNA.

In many cases, bacterial plasmids are used as the cloning vectors for the large-scale production of this specific DNA. Plasmids are small circular DNA molecules capable of replication within the host bacterium. Defining them as vectors refers to the fact that they will be used to carry the desired piece of foreign DNA. Typically, the plasmid DNA and DNA of interest are separately cleaved with identical restriction endonucleases. Products from the two reactions are mixed together in the presence of an enzyme known as DNA ligase, which facilitates rejoining. Because of the complementary nature of the DNA ends, a certain number of reannealings will result in a permanent insertion of foreign DNA into vector (plasmid) DNA. The plasmids are allowed to enter bacteria that are then cultured.

Other small DNA particles such as bacteriophages can also be used as vectors. Cloning a large number of recombinant DNA particles results in the construction of a "gene library." The gene library 
contains numerous unique but random pieces of DNA of interest. In order to isolate DNA of a certain specificity from the library, a specific probe is required. Such probes are constructed by working backwards from the protein of interest. The resulting DNA is known as complementary DNA (cDNA) since its sequences are complementary to the mRNA coding for the protein. By radioactively tagging the cDNA, one can detect which native DNA fragments contain the gene of interest.

Using such techniques, probes specific for the immunoglobulin genes and T-cell receptor genes were discovered during the early 1980 s. $^{6,119,120,261}$ One of the first clinically useful applications of this new technology was the ability to determine the clonal status of Band T-lymphocyte proliferations in cases that previously had been indeterminate, such as null cell lymphomas and PTLDs. ${ }^{6,46,49,50,264}$ Even before this, an appreciation of the fine structure of immunoglobulin gene rearrangement of B-cells led to both basic and clinically useful insights.

Normal Immunoglobulin Gene Rearrangement.-In order to understand how recombinant DNA analysis is useful in determining the clonal composition of B-cell proliferations it is necessary to have a working knowledge of the normal genetic changes that occur in these cells.

Each B-lymphocyte produces an antibody of a single specificity. In aggregate, the antibodies produced by an individual host constitute a repertoire of amazing diversity in regard to the ability to respond to different antigens. Indeed, antibodies exist for which the antigens have not yet been invented!

The basis of this antibody heterogeneity exists in the ability of the DNA to transpose, or rearrange, itself within each individual B-cell. ${ }^{150}$ The pattern of these rearrangements defines a unique nucleic acid sequence, and ultimately a unique amino acid sequence, that determines the antibody specificity of that B-cell and all its progeny. Rearrangements occur within those sections of DNA that code for the light chains and the heavy chains that compose the immunoglobulins. The light chain may be one of two types: kappa chain, coded for by chromosome 2, or lambda chain, on chromosome 22. All heavy chains (G, M, A, D, E) are coded for by a long sequence of DNA located on chromosome 14. Since each chain is coded for by several discontinuous pieces of DNA on the particular chromosome, the rearrangements are necessary to bring these pieces of DNA into apposition to form the complete gene. This process is subject to a certain degree of "looseness" at several levels, which constitutes an ingenious exploitation by nature to generate unique sequences. This looseness was predicted by Burnet almost 30 years ago in the same context (see Introduction: Section 3). 
We will examine rearrangement of the kappa gene, which is the simplest case ${ }^{150}$ (Fig 24). The complete kappa light chain is coded for by three separate regions of DNA. These are referred to as the variable region $(\mathrm{Vk})$, the joining region $(\mathrm{Jk})$, and the constant region $(\mathrm{Ck})$. First, one of the multiple $\mathrm{Vk}$ regions rearranges so as to lie next to one of the several Jk regions. The intervening DNA "loops out" and is deleted. A Vk gene now lies next to a Jk gene. This complex is still separated from the single $\mathrm{Ck}$ region by an intervening sequence of DNA. The entire length of DNA is translated onto RNA, and the in-

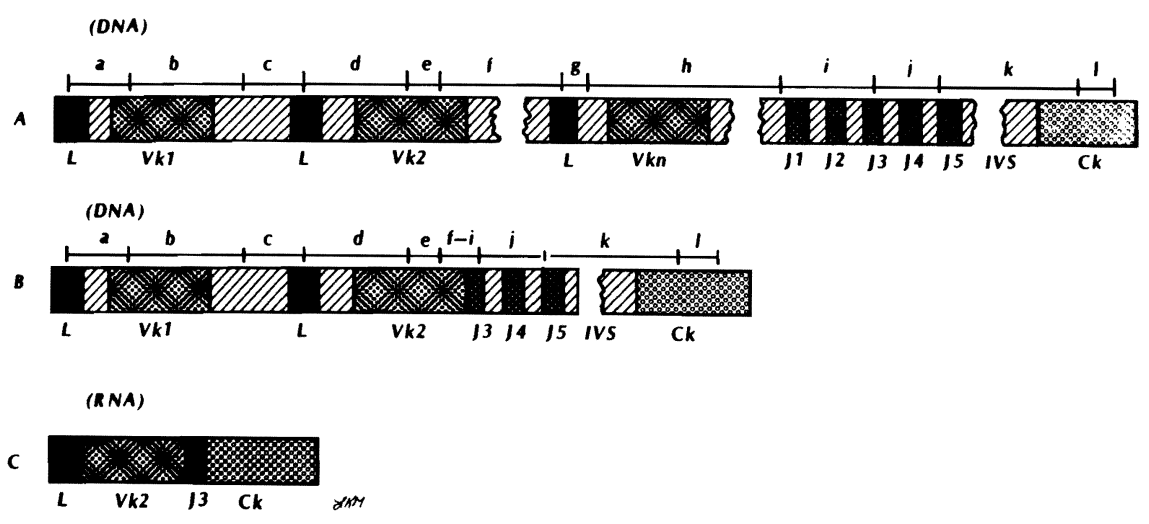

\section{FIG 24.}

Schematic representation of kappa light chain gene rearangement. In $\mathbf{A}$, the germline configuration is shown. At this point, the cell has not rearranged the kappa gene. This configuration is retained throughout life in cells other than B lymphocytes. A number of variable light chain genes $(V k 1-V k n)$ are separated by a number of DNA base pairs. Each variable gene is preceded by a leader $(L)$ sequence. There are several genes coding for the joining region $(J 1-5)$. This region joins the variable and constant regions on the immunoglobulin light chain. The joining region genes are separated from the single constant chain gene (CK) by an intervening sequence of DNA (IVS). The crossbars above the DNA represent hypothetical recognition sites of a prototypical restriction endonuclease. With digestion by the endonuclease, this DNA would fragment into separate portions $A-L$ of specific sizes, represented by the distance between crossbars. In $\mathbf{B}$, a rearrangement has occurred between one of the variable chain genes (Vk2) and one of the joining region genes (J3). All of the DNA between these segments has been lost. Therefore, fragments $f, g, h$, and $i$, which would be seen if the germline DNA in A were to be digested, are no longer generated. In their place, a single fragment (designated $f-i$ ) is seen. The other restriction fragments would remain the same, since no rearrangements have occurred in these areas. Thus fragments $a, b, c, d, e, j, k$ and $/$ would show up as germline bands, and the new fragment $f-i$ would show up as a rearranged band on Southern blot. Visualization of fragment $t-i$ requires that a sufficient (monoclonal) number of cells are present with this identical rearrangement. $\mathbf{C}$, the messenger RNA derived from this rearranged DNA. Unnecessary base pairs have been eliminated by RNA splicing, leaving only those genes necessary to directly code for the kappa light chain. 
tervening sequence is spliced out, leaving a messenger RNA with only the necessary ingredients for coding the kappa light chain.

In the case of the lambda light chain (not shown), similar rearrangements occur, although these are somewhat more complex due to inherited variability in this gene. In the case of the heavy chain rearrangements, an additional gene (Dh) codes for the "diversity" region. This gene also rearranges at an early stage in the life of the cell, further expanding the range of possible antibody specificities. ${ }^{1}$

It will be recalled that the normal cell is diploid, that is, it contains two sets, or alleles, of every gene. The immunoglobulin genes are no exception. Because the rearrangement process is so complex, mistakes are frequent. These mistakes generally result in a nonfunctional gene. Somehow this signals the cell to "try again" on the other allele. In other words, the cell gets 2 chances to make a productive kappa light chain gene. If these both fail, it then gets two chances to try with the lambda light chain gene. All of the heavy chain gene fragments are linked, so there are also two chances to construct a productive heavy chain gene. A definite hierarchy of rearrangements also exists in normal cells. ${ }^{150}$ Thus, heavy chain rearrangement occurs first. If the cell fails at this, it cannot become a functional B cell and is probably aborted. Following heavy chain rearrangement, the cell attempts to make light chains: first with the kappa genes and, if this is unsuccessful, then with the lambda genes. The result of successful gene rearrangement is the production of a complete and unique antibody molecule that will be produced by that $B$ cell and all of its progeny. The diagnostic exploitation of this phenomenon for purposes of clonal determination of B-cell proliferations will be considered next.

Use of Recombinant DNA Techniques to Assess Clonal Composition.-DNA can be cut into specific fragments by the use of restriction endonuclease enzymes, described above. When genetic rearrangements (e.g., immunogloblin gene rearrangements) occur, however, the positions of some of the endonuclease recognition sites are moved (see Fig 24). Thus the sizes of digested fragments flanked by these rearranged sites will be different from that of germline DNA.

Detecting differences in the sizes of digested DNA fragments allows us to separate those DNAs that have undergone rearrangements from those that have not. DNA can be separated by the use of gel electrophoresis. In this technique, the digested sample is placed in a gel that is cast from agarose or polyacrylamide, and an electrical current is applied to the gel (Fig 25). Conditions can be manipulated so that the specimen migrates according to molecular weight, net charge, or some combination of the two. For our purposes it is desirable to separate the digested DNA fragments by molecular weight, i.e., size, alone. 


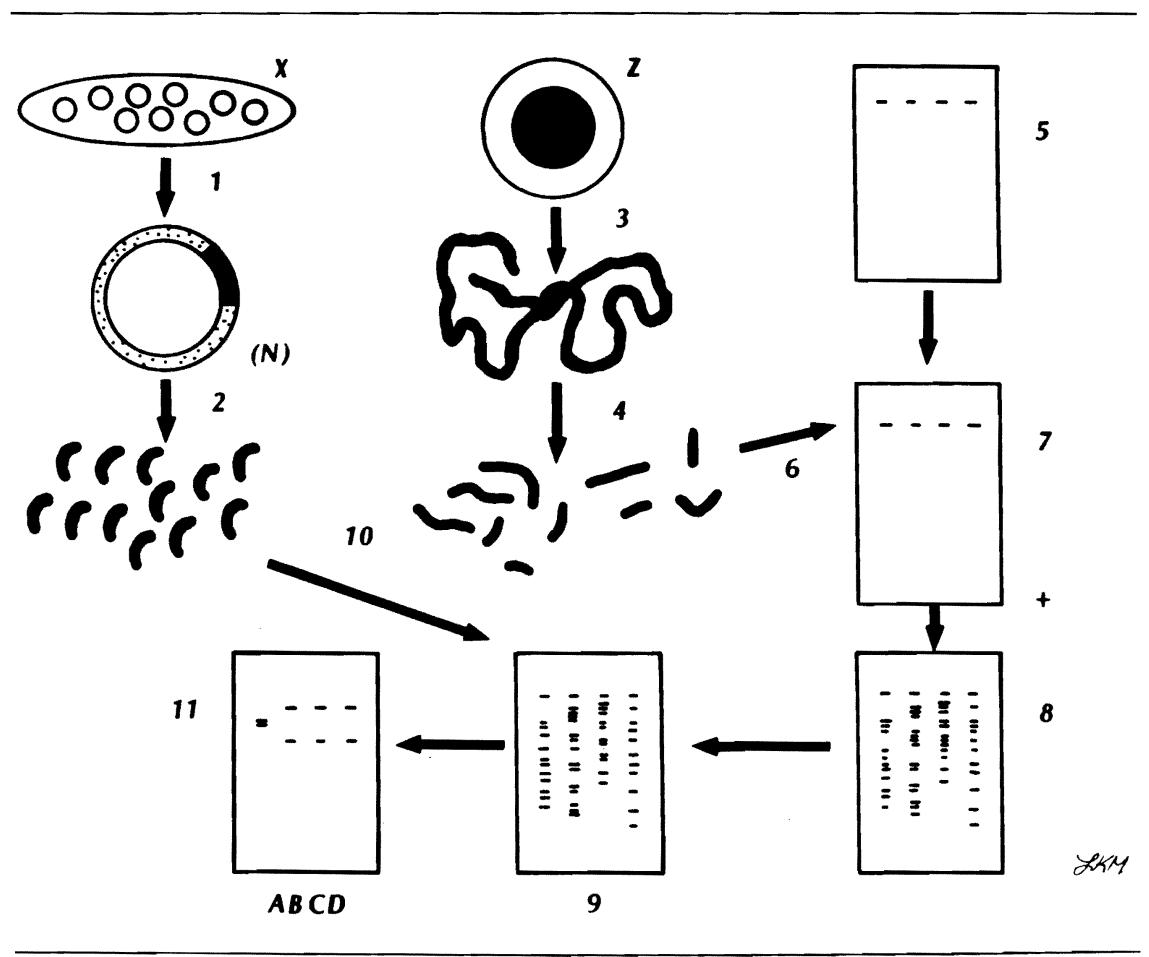

FIG 25.

Simplified flow diagram of Southern blotting for determination of immunoglobulin gene rearrangements. A bacterial host $(x)$ serves for the reproduction of plasmids (small circles) which contain a DNA probe for a portion of the immunoglobulin gene. The procedures used to insert the DNA probe into the plasmid are not shown here. The plasmids are isolated from the bacteria (1) and the DNA of interest (dark color) is separated using restriction endonucleases. This DNA is tagged with radioactive material to serve as a probe. Meanwhile, the human lymphocytes $(z)$ are disrupted, and the human DNA is isolated (3). This is cut into a number of irregularly sized pieces by restriction endonucleases (4). The DNA will be separated according to size using a gel into which small wells have been cut to hold the DNA for electrophoresis (5). Once applied to the gel (6), the DNA migrates under the influence of an electrical field (7). The gel in this diagram is drawn to contain four separate specimens, which will migrate under the same conditions. The hypothetical results of the electrophoresis are shown (8). At this point, the bands are not visible. They are displayed in (8) and (9) for the sake of clarity. In order to stablize the DNA and prevent further diffusion, the gel is blotted onto nitrocellulose paper, which produces a replicate pattern of the DNA bands (9). This paper is then incubated together with the radioactively tagged DNA probe (10), and the final product is placed on X-ray film. The resulting autoradiogram (11) will show bands only at the sites where the radioactive probe DNA has bound to the underlying human DNA and exposed the Xray film. In this hypothetical example, the similar patterns of lanes $B, C$, and $D(11)$ are used to depict a germline, or unrearranged, DNA pattern. In contrast, lane A shows a loss of the germline bands and displays two new rearranged bands. Lane A contains DNA from a monoclonal B-cell lesion, and lanes B-D contain only polyclonal lymphoid cells. 
Having done this, it is necessary to be able to localize the particular gene of interest. To do this requires the use of a specific probe, composed of DNA that is complementary in sequence to the DNA to be examined. Probes may be constructed by a variety of techniques. The probe is usually labeled with a radioactive substance such as ${ }^{32} \mathrm{P}$. Obviously the probe will bind to the DNA against which it is directed, regardless of location on the gel. In this way the size of the particular DNA fragment can be deduced when an autoradiogram of the separated DNA with bound probe is made.

During incubation steps it is helpful to immobilize the separated DNA so as to eliminate problems related to diffusion in the gel. This immobilization is achieved by blotting the gel with nitrocellulose paper. The paper absorbs and covalently bonds the DNA, thus reproducing and preserving the results of the electrophoretic separation. This approach to the analysis of DNA is referred to as Southern blotting, first described in $1975{ }^{240}$ Similar techniques, developed for the analysis of RNA and protein, have been called Northern blot and Western blot, respectively.

In the particular case of B-lymphocytes, the probes of interest are directed against either the heavy chain gene region or the kappa or lambda light chain genes. We will consider the heavy chain probe as a prototype.

In all cells with nonrearranged immunoglobulin heavy chain regions, a specific pattern, called germline, will be seen on Southern blotting. This pattern is seen in all non-B-cells and in any future Bcells that have not yet rearranged their immunoglobulin genes. In Bcells with a rearranged heavy chain gene, the rearrangement will most likely cause a change in the size of some of the fragments generated by digestion with restriction endonucleases. It is possible, of course, that a rearrangement will result in fragments in which all of the endonuclease recognition sites on the DNA happen to have the exact same number of nucleotides between them, resulting in a pattern indistinguishable from germline. However, this possibility is quite remote.

In a polyclonal (nonclonal) proliferation of B-cells, each cell will have a unique rearrangement. Thus, when subject to electrophoresis, each fragment will move to a slightly different location, depending upon size. However, each fragment will continue to label with the specific radioactive probe. The result will be a "blur" on autoradiogram, in which the pattern produced by any individual cell will be below the threshold of detection.

If, however, a monoclonal population is present in the proliferation, the DNA of each cell comprising that clone will be cut in exactly the same way, and the size will be different from the germline configuration. Therefore a distinct band pattern will emerge by 
Southern blot, and this will permit the diagnosis of a monoclonal proliferation (Fig 26) ${ }^{6,46}$

A similar approach can be used to diagnose $\mathrm{T}$-cell proliferations using a probe for the T-cell receptor. The gene for this receptor is related to the immunoglobulin gene, contains $\mathrm{V}, \mathrm{D}, \mathrm{J}$, and $\mathrm{C}$ segments, and undergoes unique rearrangements in a manner probably very similar to that of the immunoglobulin genes. ${ }^{24,123}$

Only B-lymphocytes regularly show rearrangements of heavy and light chain immunoglobulin genes. Occasional T-cell tumors may also have rearrangements of the heavy chain, and rarely the light

\section{HEAVY CHAIN GENE ANALYSIS}

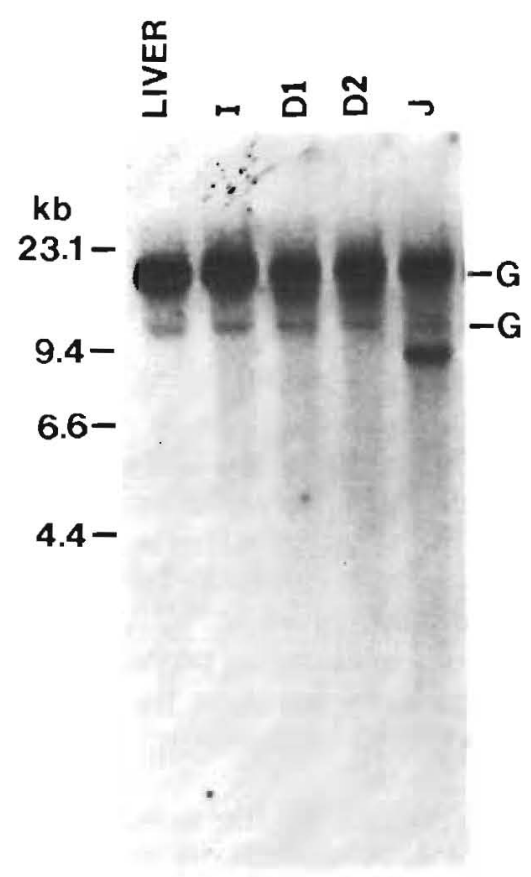

FIG 26.

Representative autoradiogram showing heavy chain immunoglobulin gene rearangement. Five separate lanes contain DNA isolated from control liver and tumors designated as I, $D, D$ and $J$. Position of DNAs of varying molecular weights in kilobases (kb) is given on scale to left. It can be seen that smaller fragments migrate further. Bands in each lane represent DNA hybridized to a radioactively labeled probe specific for a region of the heavy chain. Liver DNA serves to define the germline (unrearraniged) pattern. A similar pattern is seen in lanes I, D and D. In contrast, a rearranged band is seen in lane J. This band represents DNA from a monoclonal population of B lymphocytes. (From Nalesnik $M A$, et al: Clonal characteristics of post-transplant lymphoproliferative disorders. Transplant Proc 1988; 20(suppl 1):280-283. Reproduced with permission.) 
chain, gene in association with rearrangements of the specific T-cell receptor. Conversely, occasional B-cell tumors show rearrangement of the T-cell receptor $\beta$-chain gene in association with immunoglobulin gene rearrangements. ${ }^{6,106,183,228,265}$ Thus, whereas a heavy chain rearrangement represents conclusive proof of clonality and strongly implicates that B-lymphocyte as the cell of origin, a T-lymphocyte origin cannot be ruled out unless follow-up analyses are also performed. ${ }^{183,228}$

The immunogenotypic DNA rearrangement studies are more sensitive than the immunophenotypic immunocytochemical techniques, and they are capable of detecting a clonal component when it makes up only 5\% of the DNA analyzed. ${ }^{6}$ Other estimates have lowered this figure to $1 \%{ }^{50}$ Currently, however, the turnaround time for this procedure is on the order of several weeks, rendering it less than useful in the acute clinical setting. It can be anticipated that major progress in accelerating the procedure will be achieved in the near future.

\section{CONSIDERATIONS IN THE DIAGNOSIS OF BENIGN VERSUS MALIGNANT LYMPHOID NEOPLASMS}

\section{Introduction}

It is clinically convenient to be able to divide tumors into benign and malignant forms. In most cases this is easily accomplished, and the contemporary form of appropriate therapy is undertaken by the physician. However, the distinction between benign and malignant proliferations is made less clear by experimental carcinogenesis research throughout this century, which has led to the conclusion that cancer is a multistep process. ${ }^{53,54,63,72,87-89,143,147,156,216,217,225}$ Similar to the replacement of classical physics by quantum mechanics in the first half of the 20th century, the results of this research do not overthrow our clinical understanding of cancer, but simply expand it.

It is likely that most cancers, by the time of their clinical appearance, have evolved to a fully malignant state. Other growths (e.g., fibroadenoma) are detected during a prolonged benign period that may extend for the lifetime of the host. A limited number of tumors have behaviors somewhat intermediate between these two categories and usually are stubbornly recurrent. A fourth category may show a rapidly progressive and finely graded progression from benign to malignant. It is into this last category that PTLD appears to fall. For this reason, complete understanding of these tumors may ultimately prove to have importance well beyond its immediate applicability to this highly restricted group of patients. 


\section{Tumor Progression}

A terminology for the natural history of cancer was developed on the basis of animal experiments and clinical observations conducted during the early and middle parts of this century. This terminology is independent of the particular type of cancer and is used to codify various stages of preneoplasia and neoplasia at a cellular level.

On the basis of studies of skin cancer resulting from the application of tars to rabbit skin, Rous and colleagues ${ }^{87,89,217}$ distinguished two antecedents of neoplasia, namely cellular "initiation" and "promotion." A stimulus capable of initiation caused an irreversible cellular response that resulted in the production of latent tumor cells. A tumor promoter caused hyperplasia of the affected cells, probably along with a delay in maturation. In contrast to initiation, promotion was a reversible event and could subside following withdrawal of the stimulus.

Further growth of the tumor was dependent on a process called tumor "progression." This concept was initiated by the work of Rous, ${ }^{216}$ promoted by the principles of Foulds, ${ }^{87,88}$ and promulgated and extended by the Kleins. ${ }^{142-144}$ It proposes that the structure and behavior of tumors are composed of particular unit characters. These units may progress independently in a continuous or discontinuous manner along alternate paths of development. The units control rate of growth, differentiation, metastatic potential, and other characteristics. This model predicts that multiple tumors in the same host may progress independently of each other. It also asserts that a neoplasm most likely will not fully evolve during the lifetime of the host. In other words, given enough time, it will become even more malignant.

This concept also has implications for the beginning stages of tumor development. In the words of Foulds, "Malignancy is essentially a clinical concept . . . A tumor may have one or more of the recognized criteria of malignancy; it may have all of them and kill the patient and yet be responsive and 'conditional'." tional was first used by Rous ${ }^{217}$ to refer to neoplastic growths that were dependent upon outside aid (i.e., tar administration in his animal model) for continued survival.

In discussing PTLD we will develop the case that many of these tumors represent conditional neoplasias. We define conditional neoplasia in the current context as a neoplastic proliferation of cells that is normally forbidden by the immunocompetent host. In the immunodeficient individual, however, the "outside aid" is supplied by the removal of host control mechanisms. The tumors may therefore act as clinically aggressive malignancies that can rapidly lead to death. The pathologic and clinical spectrums of PTLDs also suggest that they may undergo tumor progression in the classic sense outlined above. 


\section{Oncogenes}

The concept of oncogenes, or cancer genes, derived from two initially unrelated fields of study. The first was the analysis of the composition of viruses capable of inducing neoplasms in susceptible animals, (acutely transforming retroviruses) and the second area was the study of the effect that DNA fragments derived from tumors had when inserted into certain cell culture systems (DNA transfection studies of NIH 3T3 fibroblasts). ${ }^{11}$

In both cases, certain genes were isolated that appeared to confer neoplastic potential on eukaryotic cells. In viruses these sequences were known as the v-onc genes. They were thought to originate in and be derived from the cellular homologs, or c-onc genes. ${ }^{8} \mathrm{C}$-onc genes have been significantly conserved throughout evolution and are thought to play significant, but poorly characterized, roles in normal cell growth and differentiation. The more than 20 known oncogenes have been tentatively divided into several families based on their known characteristics. Since the oncogene products are normal cellular constituents, their products would not normally be expected to induce a host immune response. ${ }^{141}$ However, some DNA viruses produce their own transforming proteins, which may be antigenic to the host.

Deregulation of the production of oncogene products is thought to play' a significant role in the progression of neoplastic potential Several lines of investigation have indicated that the effects of the deregulation of 2 or more oncogenes may be complementary and additive as regards the malignant phenotype ${ }^{20,63}$ (However, more recent studies have indicated that the action of a single oncogene may be enhanced by multiple abnormalities within it, abrogating the necessity for a second oncogene. ${ }^{20,63}$ Thus, to some degree, the oncogenes provide basic building blocks in constructing the "unit characteristics" of progressive neoplasia first elaborated by Foulds.

Oncogenes may be inappropriately activated by a number of mechanisms. ${ }^{8,20}$ In the case of the acutely transforming retroviruses, active oncogenes are transmitted to many cells by the virus vector, causing polyclonal tumors. Cellular oncogenes may be inappropriately activated by coming under the influence of a viral promoter, or by a genetic recombination in which the normally silent oncogene is rearranged to become part of an active gene. Actual duplications, or amplification of the oncogene, may occur, leading to increased product. Additionally, a mutation in the oncogene may code for a protein that provides an aberrant growth signal. ${ }^{11}$ Finally, one must consider the possibility that inactivation of an oncogene suppressor mechanism may be the initial event leading to increased oncogene expression., ${ }^{8,143}$

The c-myc oncogene has particular relevance to Burkitt's lym- 
phoma. ${ }^{58-60,64,143}$ Products of this gene probably play a role in normal cellular proliferation, acting together as a transducer which receives signals from growth factors and carries out functions at the level of the cell nucleus. ${ }^{220}$ The gene, which has been conserved for over 400 million years, ${ }^{220}$ is normally expressed in a coordinated fashion with other oncogenes following lymphocyte stimulation. ${ }^{156}$ Thus, disordered production of the c-myc product may help to maintain a cell in a constant state of proliferation. However, this is not thought to be a sufficient cause of malignancy in and of itself. ${ }^{162}$ Recent studies in model animal lymphoid tumors suggest that the comcomitant deregulation of c-myc with another oncogene, Blym-1, may provide the steps necessary for a malignant monoclonal lymphoproliferation to arise ${ }^{53,54} \mathrm{~A}$ similar multistep process has also been proposed for Burkitt's lymphoma. ${ }^{144}$ Recently, the copresence of EBV transformation and c-myc expression has been found to be sufficient to induce tumorigenesis in an experimental system. ${ }^{163}$

It is important to reiterate the relationship of oncogenes vis á vis the acutely transforming retroviruses and the Epstein-Barr virus. The acutely transforming retroviruses can induce neoplasia by the insertion into the host genome of oncogenes carried by the virus. The Epstein-Barr virus does not carry oncogenes that induce cancer directly. In Burkitt's lymphoma, the c-myc oncogene, a normal cellular component, is activated by chromosomal translocation. The exact cause of this translocation remains unknown. Recent reports have also suggested that EBV infection may also favor activation of other cellular oncogenes. ${ }^{206,254}$ The significance of this remains to be defined.

\section{REFERENCES}

1. Alessandro A, Pierce $\mathrm{JH}$, Baltimore $\mathrm{D}$, et al: Continuing rearrangement of immunoglobulin and T-cell receptor genes in a Ha-ras-transformed lymphoid progenitor cell line. Proc Natl Acad Sci USA 1987; 84:1799-1803.

2. Alsip G, Ench Y, Sumaya C, et al: Detection of Epstein-Barr virus DNA in saliva of patients with AIDS and related disorders, in Levine PH, Ablashi DV, Nonoyama M, et al (eds): Epstein-Barr Virus and Human Disease. Clifton, NJ, Humana Press, 1987, pp 119-120.

3. Anderson JL, Fowles RE, Bieber CP, et al: Idiopathic cardiomyopathy, age, and suppressor-cell dysfunction as risk determinants of lymphoma after cardiac transplantation. Lancet 1978; 2:1174-1177.

4. Andiman W, Gradoville L, Heston $\mathrm{L}$, et al: Use of cloned probes to detect Epstein-Barr viral DNA in tissues of patients with neoplastic and lymphoproliferative disease. $J$ Infect Dis 1983; 148:967-977.

5. Armstrong G, Longo D, Faggioni A, et al: Detection and isolation of Epstein-Barr virus in lymphocytes from patients with chronic B-lymphocyte leukemia, in Magrath IT, O'Conor GT, Ramot B (eds): Pathogenesis of Leukemias and Lymphomas: Environmental Influences, New York, Raven Press, 1984, pp 259-262. 
6. Arnold A, Cossman J, Bakshi A, et al: Immunoglobulin-gene rearrangements as unique clonal markers in human lymphoid neoplasms. $N$ Engl J Med 1983; 309:1593-1599.

7. Aronson FR, Dempsey RA, Allegretta M, et al: Malignant granular lymphoproliferation after Epstein-Barr virus infection: Partial immunologic reconstitution with interleukin-2. Am J Hematol 1987; 25:427-439.

8. Astrin SM, Rovigatti UG: Oncogene expression in human lymphoid neoplasms, in Magrath IT, O'Conor GT, Ramot B (eds): Pathogenesis of Leukemias and Lymphomas: Environmental Influences, New York, Raven Press, 1984, pp 349-357.

9. Audouin J, Tourneau AL, Aubert JP, et al: Herpes simplex virus lymphadenitis mimicking tumoral relapse in a patient with Hodgkin's disease in remission. Virchows Arch $[A]$ 1985; 408:313-321.

10. Baer R, Bankier AT, Biggin AT, et al: DNA sequence and expression of the B95-8 Epstein-Barr virus genome. Nature 1984; 310:207-211.

11. Bartram CR: Activation of proto-oncogenes in human leukemias. Blut 1985; 51:63-71.

12. Beveridge $T$, Krupp P, McKibbin C: Lymphomas and lymphoproliferative lesions developing under Cyclosporin therapy. Letter. Lancet 1984; 1:788.

13. Bia MJ, Flye MW: Brief communication: Immunoblastic lymphoma in a cyclosporine-treated renal transplant recipient. Transplantation 1985; 39:673-675.

14. Bieber CP, Reitz BA, Jamieson SW, et al: Malignant lymphoma in cyclosporin A treated allograft recipients. Letter. Lancet 1980; 1:43.

15. Bird AG, Britton S: The relationship between Epstein-Barr virus and lymphoma. Sem Hematol 1982; 19:285-300.

16. Bird AG, McLachlan SM: Cyclosporin A and Epstein-Barr virus. Lancet $1980 ; 2: 418$.

17. Bird AG, McLachlan SM, Britton S: Cyclosporin A promotes spontaneous outgrowth in vitro of Epstein-Barr virus-induced B-cell lines. Nature 1981; 289:300-301.

18. Birkeland SA: Cancer in transplanted patients: The Scandia Transplant material. Transplant Proc 1983; 15:1071-1078.

19. Birkeland SA: Immune monitoring of tumor development after renal transplantation. Cancer 1985; 55:988-994.

20. Bishop JM: The molecular genetics of cancer. Science 1987; 235:305-311.

21. Bloom RE, Brennan JK, Sullivan JL, et al: Lymphoma of host origin in a marrow transplant recipient in remission of acute myeloid leukemia and receiving Cyclosporin A. Am J Hematol 1985; 18:73-83.

22. Bonmassar E, Menconi E, Goldin A, et al: Escape of small numbers of allogeneic lymphoma cells from immune surveillance. $J$ Natl Cancer Inst 1974; 53:475-479.

23. Boring CC, Brynes RK, Chan WC, et al: Increase in high-grade lymphomas in young men. Lancet 1985; 1:857-859.

24. Born W, Yague J, Palmer E, et al: Rearrangement of T-cell receptor $\beta$ chain genes during T-cell development. Proc Natl Acad Sci USA 1985; 82:29252929.

25. Brewster FE, Byron KS, Sullivan JL: Immunoregulation during acute infection with Epstein-Barr virus: Dynamics of interferon and $2^{\prime}, 5^{\prime}$-oligoadenylate synthetase activity. $J$ Infect Dis $1985 ; 151: 1109-1115$.

26. Brichacek B, Davis J, Purtilo DT: Presence of monoclonal and oligoclonal B-cell proliferation in fatal infectious mononucleosis, in Levine $\mathrm{PH}$, 
Ablashi DV, Nonoyama M, et al (eds): Epstein-Barr Virus and Human Disease. Clifton, NJ, Humana Press, 1987, pp 53-54.

27. Briggs JD, Hamilton DNH, Macsween RNM, et al: Infectious mononucleosis, herpes simplex infection, and diffuse lymphoma in a renal transplant patient. Transplantation 1978; 25:227-228.

27a. Brooks JJ: Kaposi's sarcoma: A reversible hyperplasia. Lancet 1986; 2:13091310.

28. Brown N, Smith D, Miller G, et al: Infectious mononucleosis: A polyclonal $B$ cell transformation in vivo. $J$ Infect Dis 1984; 150:517-522.

29. Brown NA, Liu C, Garcia CR, et al: Clonal origins of lymphoproliferative disease induced by Epstein-Barr virus. J Virol 1986; 58:975-978.

30. Brumbaugh J, Baldwin JC, Stinson EB, et al: Quantitative analysis of immunosuppression in Cyclosporine-treated heart transplant patients with lymphoma. Heart Transplant 1985; 4:307-311.

31. Burkitt D: Discovering Burkitt's Lymphoma, in Levine PH, Ablashi DV, Nonoyama M, et al (eds): Epstein-Barr Virus and Human Disease. Clifton NJ, Humana Press, 1987, pp xxi-xxxi.

32. Burkitt D: A sarcoma involving the jaws in African children. Br J Surg 1958; 46:218-223.

33. Burnet FM: Cancer-A biological approach. Br Med J 1957; 1:779-786.

34. Burnet FM: Evaluation of the evidence for immune surveillance: Impressions and comments, in Smith RT, Lands M (eds): Immune Surveillance. New York, Academic Press, 1970, pp 512-518.

35. Burnet FM: The concept of immunological surveillance. Prog Exp Tumor Res 1970; 13:1-27.

36. Burnet FM: Immunological surveillance in neoplasia. Transplant Rev 1971; 7:3-25.

36a. Burnet M: Immunologic Surveillance. New York, Pergamon Press, 1970, p 161.

37. Canadian Multicentre Transplant Study Group: A randomized clinical trial of Cyclosporin in cadaveric renal transplantation. N Engl J Med 1983; 309:809-815.

38. Calne RY, Rolles K, Thiru S, et al: Cyclosporine A initially as the only immunosuppressant in 34 recipients of cadaveric organs: 32 kidneys, 2 pancreases, and 2 livers. Lancet 1979; 2:1033-1036.

39. Case Records of the Massachusetts General Hospital: Weekly clinicopathological exercises. Case 42-1986. A 28 year old man with a renal transplant and recent disorientation. $N$ Engl $J$ Med 1986; 15:1079-1086.

40. Castro CJ, Klimo P, Worth A: Multifocal aggressive lymphoma of the gastrointestinal tract in a renal transplant patient treated with Cyclosporin A and Prednisone. Cancer 1985; 55:1665-1667.

41. Chang RS, Lewis JP, Reynolds RD, et al: Oropharyngeal excretion of EBV by patients with lymphoproliferative disorders and by recipients of renal homografts. Ann Intern Med 1978; 88:34.

42. Childs CC, Parham DM, Berard CW: Infectious mononucleosis: The spectrum of morphologic changes simulating lymphoma in lymph nodes and tonsils. Am J Surg Pathol 1987; 11:122-132.

43. Ciobanu N, Andreef $M$, Safai B, et al: Lymphoblastic neoplasia in a homosexual patient with Kaposi's sarcoma. Ann Intern Med 1983; 98:151-155.

44. Clark EA, Ledbetter JA: Activation of B cells mediated through two distinct cell surface differentiation antigens, Bp35 and Bp50. Proc Natl Acad Sci USA 1986; 83:4494-4498. 
45. Cleary ML, Sklar J: Lymphoproliferative disorders in cardiac transplant recipients are multiclonal lymphomas. Lancet $1984 ; 2: 489-493$.

46. Cleary $\mathrm{ML}$, Chao J, Warnke $\mathrm{R}$, et al: Immunoglobulin gene rearrangement as a diagnostic criterion of B-cell lymphoma. Proc Natl Acad Sci USA 1984; 81:593-597.

47. Cleary ML, Dorfman RF, Sklar J: Failure in immunological control of the virus infection: Post-transplant lymphomas, in Epstein MA, Achong BG (eds): The Epstein-Barr Virus: Recent advances. New York, John Wiley and Sons, 1987, pp 164-181.

48. Cleary ML, Meeker TC, Levy S, et al: Clustering of extensive somatic mutations in the variable region of an immunoglobulin heavy chain gene from a human B cell lymphoma. Cell 1986; 44:97-106.

49. Cleary ML, Trela MJ, Weiss LM, et al: Most null large cell lymphomas are B lineage neoplasms. Lab Invest 1985; 53:521-525.

50. Cleary ML, Warnke R, Sklar J: Monoclonality of lymphoproliferative lesions in cardiac-transplant recipients. $N$ Engl J Med 1984; 310:477-482.

51. Clift S, Lanier A, Kline $\mathrm{K}$, et al: Evidence for association of Epstein-Barr virus with malignant lymphoepithelial lesion of salivary gland, in Levine PH, Ablashi DV, Nonoyama M, et al (eds): Epstein-Barr Virus and Human Disease. Clifton NJ, Humana Press, 1987, pp 79-83.

52. Colby B, Shaw J, Elion G, et al: Effect of Acyclovir [9-(2-hydroxyethoxymethyl)guanine] on Epstein-Barr virus DNA replication. J Virol 1980; 34:560568.

52a. Coons AH, Creech HJ, Jones RN, et al: Demonstration of pneumococcal antigen in tissues by use of fluorescent antibody. J Immunol 1942; 45:159170.

53. Cooper GM: Molecular and Functional analysis of ras and Blym transforming genes, in Wahren B (ed): Molecular Biology of Tumor Cells. New York, Raven Press, 1985, pp 1-7.

54. Cooper MD, Bertoli LF, Borzillo GV, et al: Pathogenesis of B cell malignancies, in Gale RP, Golde DW (eds): Leukemia: Recent Advances in Biology and Treatment. New York, Alan R Liss, Inc, 1985, pp 453-465.

55. Cotton JR, Sarles AG, Remmers AR, et al: The appearance of reticulum cell sarcoma at the site of antilymphocyte globulin injection. Transplantation $1973 ; 16: 154-157$.

56. Crawford DH, Thomas JA, Janossy G, et al: Epstein-Barr virus nquclear antigen positive lymphoma after Cyclosporin A treatment in patient with renal allograft. Letter. Lancet 1980; 1:1355-1356.

57. Crawford DH, Weller I, Iliescu V: Polyclonal activation of B cells in homosexual men. Letter and reply. $N$ Engl J Med 1984; 311:536-537.

58. Croce CM, Nowell PC: Molecular basis of human B cell neoplasia. Blood $1985 ; 65: 1-7$.

59. Croce CM, Erikson J, Tsujimoto $\mathrm{Y}$, et al: Molecular basis of human B- and T-cell neoplasia. Adv Viral Oncol 1987; 7:35-51.

60. Croce CM: Minireview: Role of chromosome translocations in human neoplasia. Cell 1987; 49:155-156.

61. Cunningham TR, Ascher NL: Effect of prerenal transplant OKT4-OKT8 ratio and immunosuppression protocol on posttransplant viral infections. Transplant Proc 1985; 17:633-634.

62. Cunningham-Rundles C, Siegal FP, Cunningham-Rundles S, et al: Incidence of cancer in 98 patients with common varied immunodeficiency. $J$ Clin Immunol 1987; 7:294-299. 
63. Cuzin F, Meneguzzi G: Stepwise transformation and cooperative interactions involving oncogenes of DNA tumor viruses. Adv Viral Oncol 1987; 6:21-43.

64. Dalla-Favera R, Bregni M, Erikson J, et al: Human c-myc onc gene is located on the region of chromosome 8 that is translocated in Burkitt lymphoma cells. Proc Natl Acad Sci USA 1982; 79:7824-7827.

65. Davis J, Brichacek B, Purtilo DT: Development of two monoclonal B-cell lymphomas in a patient with Wiskott-Aldrich syndrome, in Levine $\mathrm{PH}$, Ablashi DV, Nonoyama $M$ et al: Epstein-Barr Virus and Human Disease Clifton NJ, Humana Press, 1987, pp 117-118.

66. Davis MM: Molecular genetics of the T cell-receptor beta chain. Ann Rev Immunol 1985; 3:537-560.

67. De Boer RJ, Hogeweg P: Tumor escape from immune elimination: Simplified precursor bound cytotoxicity models. $J$ Theor Biol 1985; 113:719-736.

68. Demetris AJ, Nalesnik MA, Kunz HW, et al: Sequential analyses of the development of lymphoproliferative disorders in rats receiving Cyclosporine. Transplantation 1984; 38:239-246.

69. Denny CT, Hollis GF, Magrath IT, et al: Burkitt lymphoma as a model of cancer associated chromosomal translocation, in Gale RP, Golde DW (eds): Leukemia: Recent Advances in Biology and Treatment. New York, Alan R Liss, Inc, 1985, pp 107-112.

70. Deodhar SD, Kuklinca AG, Vidt DG, et al: Development of reticulum cell sarcoma at the site of antilymphocyte globulin injection in a patient with renal transplant. $N$ Engl J Med 1969; 280:1104-1106.

71. DeWaele M, Thielemans C, Van Camp BKG: Characterization of immunoregulatory $\mathrm{T}$ cells in EBV-induced infectious mononucleosis by monoclonal antibodies. $N$ Engl J Med 1981; 304:460-462.

72. Diamond A, Cooper GM, Ritz J, et al: Identification and molecular cloning of the human B-lym transforming gene activated in Burkitt's lymphomas. Nature 1983; 305:112-116.

73. Dinarello CA, Mier JW: Current Concepts: Lymphokines. $N$ Engl J Med 1987; 317:940-945.

74. Dorfman RF, Warnke R: Lymphadenopathy simulating the malignant lymphomas. Hum Pathol 1974; 5:519-550.

74a. Dorfman RF: Kaposi's sarcoma revisited. Hum Pathol 1984; 15:1013-1017.

75. Dosseter JB, Kovithavongs T, Salkie M, et al: Cyclosporine-associated lymphoproliferation, despite controlled Cyclosporine blood concentrations, in a renal allograft recipient. Proc Eur Dial Transplant Assoc Eur Ren Assoc 1984 ; 21:1021-1025.

76. Doyle TJ, Venkatachalam KK, Maeda K, et al: Hodgkin's disease in renal transplant recipients. Cancer 1983; 51:245-247.

77. Dummer JS, Bound LM, Singh G, et al: Epstein-Barr virus-induced lymphoma in a cardiac transplant recipient. Am J Med 1984; 77:179-183.

78. Lymphoma in organ transplant recipients. Editorial. Lancet 1984; 1:601603.

79. Ehlin-Henriksson B, Klein G: Distinction between Burkitt lymphoma subgroups by monoclonal antibodies: Relationships between antigen expression and type of chromosomal translocation. Int $J$ Cancer 1984; 33:459-463.

80. Eklund B, von Willebrand E, Hockerstedt $\mathrm{K}$, et al: Lymphoproliferative disease after renal transplantation. Transplant Proc 1986; 18:95-97.

81. Epstein MA, Achong BG, Barr YM: Preliminary communication: Virus par- 
ticles in cultured lymphoblasts from Burkitt's lymphoma. Lancet 1964; 1:702-703.

82. Erisman MD, Rothberg PG, Diehl RE, et al: Deregulation of c-myc gene expression in human colon carcinoma is not accompanied by amplification or rearrangement of the gene. Mol Cell Biol 1985; 5:1969-1976.

83. Faggioni A, Zompetta C, Grimaldi S, et al: Calcium modulation activates Epstein-Barr virus genome in latently infected cells. Science 1986; 232:1554-1556.

84. Farthing MJG, Clark ML: Nature of the toxicity of Cyclosporine A in the rat. Biochem Pharmacol 1981; 30:3311-3316.

85. Filipovich AH, Zerbe D, Spector BD, et al: Lymphomas in persons with naturally occurring immunodeficiency disorders, in Magrath IT, O'Conor GT, Ramot B (eds): Pathogenesis of Leukemias and Lymphomas: Environmental Influences. New York, Raven Press, 1984, pp 225-234.

86. Finch SC: Ionizing radiation and drugs in the pathogenesis of lymphoid neoplasia, in Magrath IT, O'Conor GT, Ramot B (eds): Pathogenesis of Leukemias and Lymphomas: Environmental Influences. New York, Raven Press, 1984, pp 207-223.

87. Foulds L: The experimental study of tumor progression: A review. Cancer Res $1954 ; 14: 327-339$.

88. Foulds L: The natural history of cancer. J Chronic Dis 1958; 8:2-37.

89. Friedewald WF, Rous $P$ : The initiating and promoting elements in tumor production. An analysis of the effects of tar, benzpyrene, and methylcholanthrene on rabbit skin. J Exp Med 1944; 80:101-126.

90. Frizzera G, Hanto DW, Gajl-Peczalska KJ, et al: Polymorphic diffuse B-cell hyperplasias and lymphomas in renal transplant recipients. Cancer Res $1981 ; 41: 4262-4279$.

91. Frizzera G, Rosai J, Dehner LP, et al: Lymphoreticular disorders in primary immunodeficiencies: New findings based on an up-to-date histologic classification of 35 cases. Cancer 1980; 46:692-699.

92. Fung JJ, Markus BH, Gordon RD, et al: Impact of ORTHOCLONE OKT3 on liver transplantation. Transplant Proc 1987; 14(Suppl 1):37-44.

93. Futrell JW: Transplantation tumors and immunologically privileged sites. Letter. JAMA 1972; 220:1130.

94. Gatti RA, Good RA: Occurrence of malignancy in immunodeficiency disease, in Lynch HT (ed): Cancer Genetics. Springfield, Ill, Charles C Thomas, 1976, pp 63-75.

95. Gatti RA, Good RA: Occurrence of malignancy in immunodeficiency diseases. A literature review. Cancer 1971; 28:89-98.

96. Geis WP, Iwatsuki S, Molnar Z, et al: Pseudolymphoma in renal allograft recipients (and discussion). Arch Surg 1978; 113:461-466.

97. Goldstein G: Overview of the development of ORTHOCLONE OKT3: Monoclonal antibody for therapeutic use in transplantation. Transplant Proc 1987; 14(Suppl 1):1-6.

98. Good RA: Relations between immunity and malignancy. Proc Natl Acad Sci USA 1972; 69:1026-1032.

99. Gordon $\mathrm{RD}$, Iwatsuki $\mathrm{S}$, Esquivel $\mathrm{CO}$, et al: Progress in liver transplantation. Adv Surg 1987; 21:49-64.

100. Gossett TC, Gale RP, Fleischman $\mathrm{H}$, et al: Immunoblastic sarcoma in donor cells after bone marrow transplantation. N Engl $J$ Med 1979; 300:904-907.

100a. Goto T, Kino T, Hatanaka H, et al: Discovery of FK-506, a novel immunosuppressant isolated from Streptomyces tsukabaensis. Transplant Proc 1987; 19(5 Suppl 6):4-8. 
101. Gowing NFC: Infectious mononucleosis: Histopathologic aspects. Pathol Annu 1975; 10:1-20.

102. Gross V, Thomas FT, Ganghoff $\mathrm{O}$, et al: Differential effects of immunosuppressive agents on $\mathrm{T}$ cell and $\mathrm{T}$ cell subset levels measured by monoclonal antibodies and automated flow cytometry. Transplant Proc 1985; 17:642643.

103. Gupta AK, Cardella CJ, Haberman HF: Cutaneous malignant neoplasms in patients with renal transplants. Arch Dermatol 1986; 122:1288-1293.

104. Gussander E, Adams A: Electron microscopic evidence for replication of circular Epstein-Barr virus genomes in latently infected Raji cells. $J$ Virol 1984; 52:549-556.

105. Guttmann RD, Meakins JL: Association of anergy and the development of cancer in immunosuppressed renal allograft recipients. Transplant Proc 1984; 16:495-498.

106. Ha $K$, Minden $M$, Hozumi $N$, et al: Immunoglobulin $\mu$-chain gene rearrangement in a patient with $\mathrm{T}$ cell acute lymphoblastic leukemia. $J$ Clin Invest 1984; 73:1232-1236.

107. Hanto DW, Najarian JS: Advances in the diagnosis and treatment of EBVassociated lymphoproliferative diseases in immunocompromised hosts. $J$ Surg Oncol 1985; 30:215-220.

108. Hanto DW, Frizzera G, Gajl-Peczalska J, et al: Epstein-Barr virus, immunodeficiency, and B-cell lymphoproliferation. Transplantation 1985; 39:461-472.

109. Hanto DW, Frizzera G, Gajl-Peczalska KJ, et al: Acyclovir therapy of Epstein-Barr virus-induced posttransplant lymphoproliferative disorders. Transplant Proc 1985; 17:89-92.

109a. Hanto DW, Frizzera G, Gajl-Peczalska KJ, et al: Epstein-Barr virus-induced B-cell lymphoma after renal transplantation. Acyclovir therapy and transition from polyclonal to monoclonal B-cell proliferation. $N$ Engl $J$ Med 1982; 306:913-918.

110. Hanto DW, Frizzera G, Gajl-Peczalska J, et al: The Epstein-Barr virus (EBV) in the pathogenesis of posttransplant lymphomas. Transplant Proc 1981; 13:756-760.

111. Hanto DW, Simmons RL, Najarian JS: Epstein-Barr virus-induced lymphoproliferative diseases in renal allograft recipients. Heart Transplant 1984; 3:121-131.

112. Harris, NL: Lymphoma 1987. An interim approach to diagnosis and classification. Pathol Annu 1987; 22:1-67.

113. Нӓугу $P$, von Willebrand $E$ : The influence of the pattern of inflammation and administration of steroids on Class II MHC antigen expression in renal transplants. Transplantation 1986; 42:358-363.

114. Henle G, Henle W, Diehl V: Relation of Burkitt's tumor-associated Herpestype virus to infectious mononucleosis. Proc Natl Acad Sci USA 1968; 59:94-101.

115. Henle W, Henle G: Epstein-Barr virus-specific serology in immunologically compromised individuals. Cancer Res 1981; 41:4222-4225.

116. Henle W, Henle GE, Horwitz CA: Epstein-Barr specific diagnostic tests in infectious mononucleosis. Hum Pathol 1974; 5:551-565.

117. Henle W, Henle G: Epstein-Barr virus and human malignancies. Adv Viral Oncol 1985; 5:201-238.

118. Hermans PE, Diaz Buxo JA, Stobo JD: Review: Idiopathic late onset immunoglobulin deficiency: Clinical observations in 50 patients. Am J Med 1976; 61:221-237. 
119. Hieter PA, Hollis GF, Korsmeyer SJ, et al: Clustered arrangement of immunoglobulin lambda constant region genes in man. Nature 1981; 294:536-540.

120. Hieter PA, Max EE, Seidman JG, et al: Cloned human and mouse kappa immunoglobulin constant and $\mathrm{J}$ region genes conserve homology in functional segments. Cell 1980; 22:197-207.

121. Ho M, Miller G, Atchison RW, et al: Epstein-Barr virus infections and DNA hybridization studies in posttransplantation lymphoma and lymphoproliferative lesions: The role of primary infection. J Infect Dis 1985; 152:876886.

122. Hockberg FH, Miller G, Schooley RT, et al: Central nervous system lymphoma related to Epstein-Barr virus. $N$ Engl J Med 1983; 309:745-748.

123. Hood L, Kronenberg $M$, Hunkapiller T: Review: $T$ cell antigen receptors and the immunoglobulin supergene family. Cell 1985; 40:225-229.

124. Hoover R, Fraumeni JF: Risk of cancer in renal transplant recipients. Lancet $1973 ; 2: 55-57$.

125. Hoover R: Effects of drugs-immunosuppression, in Hiatt $\mathrm{HH}$, Watson JD, Winsten JD (eds): Cold Spring Harbor Conference on Cell Proliferation. V4: Origins of Human Cancer. Cold Spring Harbor, New York, 1977, pp 369379.

126. Howard $M$, Stein $P$, Dubois $P$ : Lymphokine regulation of polyclonal and antigen-specific B cell responses, in Kripke ML, Frost $\mathbf{P}$ (eds): $M D$ Anderson Symposium on Fundamental Cancer Research, vol 38. Immunology and Cancer. Houston, University of Texas System Cancer Center, 1986, 35-42.

127. Ioachim HL: Neoplasms associated with immune deficiencies. Pathol Annu 1987; 22:177-222.

128. Iwatsuki S, Geis WP, Molnar Z, et al: Systemic lymphoblastic response to antithymocyte globulin in renal allograft recipients. An initial report. $J$ Surg Res 1978; 24:428--434.

129. Jamieson NV, Thiru S, Calne RY, et al: Brief communication: Gastric lymphomas arising in two patients with renal allografts. Transplantation 1981; 31:224-225.

130. Jondal M, Klein G: Surface markers on human B and T lymphocytes. II. Presence of Epstein-Barr virus receptors on B lymphocytes. J Exp Med 1973; 38:1365-1377.

131. Jones JF, Ray CG, Minnich LL, et al: Evidence for active Epstein-Barr virus infection in patients with persistent, unexplained illness: Elevated antiearly antigen antibodies. Ann Intern Med 1985; 102:1-7.

132. Jones JF, Shurin S, Abramowsky C, et al: T-cell lymphomas in patients with chronic EBV infection, in Levine PH, Ablashi DV, Nonoyama M, et al (eds): Epstein-Barr Virus and Human Disease. Clifton NJ, Humana Press, 1987, pp 97-100.

133. Kaplan J, Shope TC: Natural killer cells inhibit outgrowth of autologous Epstein-Barr virus-infected B lymphocytes. Nat Immun Cell Growth Regul $1985 ; 4: 40-47$.

134. Kaslikova J, Kocandrle V, Zastava V, et al: Brief communication: Multiple immunoblastic sarcoma of the small intestine following renal transplantation. Transplantation 1981; 31:481-482.

135. Keown PA, Stiller CR: Control of rejection of transplanted organs. Adv Intern Med 1987; 31:17-46.

136. Kersey JH, Spector BD, Good RA: Primary immunodeficiency diseases and cancer: The Immunodeficiency-Cancer Registry. Int $J$ Cancer 1973; 12:333347. 
137. Kinlen LJ, Hoover RN: Short communication: Lymphomas in renal transplant recipients: A search for clustering. $B r J$ Cancer 1979; 40:798-801.

138. Kinlen LJ, Webster ADB, Bird AG, et al: Prospective study of cancer in patients with hypogammaglobulinemia. Lancet 1985; 1:263-266.

139. Kishimoto T: Factors affecting B-cell growth and differentiation. Ann Rev Immunol 1985; 3:133-157.

140. Klein E, Ernberg I, Masucci MG, et al: T-cell response to B-cell and Epstein-Barr virus antigens in infectious mononucleosis. Cancer Res 1981; 41:4210-4215.

141. Klein E: Host response to Epstein-Barr virus infected B cells, in Reif AE, Mitchell MS (eds): Immunity to Cancer. Orlando, Academic Press, 1985, pp 133-148.

142. Klein G, Klein E: Immune surveillance against virus-induced tumors and nonrejectability of spontaneous tumors: Contrasting consequences of host versus tumor evolution. Proc Natl Acad Sci USA 1977; 74:2121-2125.

143. Klein G, Klein E: The evolution of independence from specific growth stimulation and inhibition in mammalian tumour-cell populations. Symp Soc Exp Biol 1957; 11:305-328.

144. Klein G, Klein E: Evolution of tumours and the impact of molecular oncology. Nature 1985; 315:190-195.

145. Klein G: Tumor Immunology. Transplant Proc 1973; 5:31-41.

146. Klein G: Herpesviruses and oncogenesis. Proc Natl Acad Sci USA 1972; 69:1056-1064.

147. Klein G: Preface, in Adv Viral Oncol 1987; 6.v-viii.

148. Klein G: Immunological surveillance against neoplasia. Harvey Lect 19734; 69:71-102.

149. Klintmalm LO, Ringden O: Mutual interaction between Prednisolone and Cyclosporine in renal transplant patients. Transplant Proc 1985; 17:12521255.

149a. Kohler G, Milstein C: Continuous cultures of fused cells secreting antibody of predefined specificity. Nature $1975 ; 256: 495-497$.

150. Korsmeyer SJ, Waldmann TA: Immunoglobulin gene rearrangement and translocation in human lymphoid malignancy. $J$ Clin Immunol 1984; 4:111.

151. Krikorian JG, Portlock CS, Cooney DP, et al: Spontaneous regression of non-Hodgkin's lymphoma: A report of nine cases. Cancer 1980; 46:20932099.

152. Krueger TC, Tallent MB, Richie RE, et al: Neoplasia in immunosuppressed renal transplant patients: A 20-year experience. South Med J 1985; 78:501506.

153. Kunkl A, Klaus GGB: Selective effects of cyclosporine A on functional B cell subsets in the mouse. $J$ Immunol 1980; 125:2526-2531.

154. Lange B, Henle W, Meyers JD, et al: Epstein-Barr virus-related serology in marrow transplant recipients. Int $J$ Cancer 1980; 26:151-157.

155. Laroye GJ: Immunologic surveillance against cancer: Critique of an established hypothesis Med Hypotheses 1975; 1:43-50.

156. Lenoir GM, Bornkamm GW: Burkitt's lymphoma, a human cancer model for the study of the multistep development of cancer: Proposal for a new scenario. Adv Viral Oncol 1987; 7:173-206.

157. Levine AM, Meyer PR, Begandy MK, et al: Development of B-cell lymphoma in homosexual men. Clinical and immunologic findings. Ann Intern Med 1984; 100:7-13.

158. Levy N, Nelson J, Meyer $P$, et al: Reactive lymphoid hyperplasia with single 
class (monoclonal) surface immunoglobulins. Am J Clin Pathol 1983; 80:300-308.

159. Levy R, Meeker T, Lowder J, et al: The immunobiology of B cell lymphoma: Clonal heterogeneity as revealed by anti-idiotype antibodies and immunoglobulin gene probes, Symposium on Fundamental Cancer Research, vol 38. Houston, University of Texas System Cancer Center, 1986, pp 261-268.

160. Linder J, Purtilo DT: Infectious mononucleosis and complications, in Epstein MA, Achong BG: The Epstein-Barr Virus: Recent Advances. New York, John Wiley and Sons, 1986; pp 11-35.

161. Lipinski M, Tetaud C, Talbot M, et al: Detection in normal tonsils of a novel B-lymphocyte population with a Burkitt-like phenotype, in Levine PH, Ablashi DV, Nonoyama M, et al (eds): Epstein-Barr Virus and Human Disease. Clifton NJ, Humana Press, 1987, pp 121-122.

162. Lippman SM, Grogan TM, Carry $P$, et al: Post-transplantation T cell lymphoblastic lymphoma. Am J Med 1987; 82:814-816.

163. Lombardi L, Newcomb EW, Dalla-Favera R: Pathogenesis of Burkitt's lymphoma: Expression of an activated c-myc oncogene causes the tumorigenic conversion of EBV-infected human B lymphoblasts. Cell 1987; 49:161-170.

164. Lukes RJ, Tindle BH, Parker JW: Reed-Sternberg-like cells in infectious mononucleosis. Letter. Lancet 1969; 2:1003-1004.

165. Makowka L, Nalesnik MA, Stieber A, et al: Control of post-transplant lymphoproliferative disorders and Kaposi's sarcoma by modulation of immunosuppression, in Good RA (ed): Nature, Cellular and Biochemical Bases, and Management of Immunodeficiency. Stuttgart-New York, FK Schattauer Verlag, in press.

166. Manolov G, Manolova Y: Marker band in one chromosome 14 from Burkitt lymphomas. Nature 1972; 237:33-34.

167. Marker SC, Ascher NL, Kalis JM, et al: Epstein-Barr virus antibody responses and clinical illness in renal transplant recipients. Surgery 1979; $85: 433-440$.

168. Martin PJ, Shulman HM, Schubach WH, et al: Fatal Epstein-Barr virusassociated proliferation of donor B cells after treatment of acute graftversus-host disease with a murine anti-T-cell antibody. Ann Intern Med $1984 ; 101: 310-315$.

169. Matas AJ, Hertel BF, Rosai J, et al: Post-transplant malignant lymphoma: Distinctive morphologic features related to its pathogenesis. Am J Med 1976; 61:716-720.

170. Matas AJ, Simmons RL, Najarian JS: Hypothesis: Chronic antigenic stimulation, herpesvirus infection, and cancer in transplant recipients. Lancet 1975; 1:1277-1279.

171. Matsuo T, Heller M, Petti L, et al: Persistence of the entire Epstein-Barr virus genome integrated into human lymphocyte DNA. Science 1984; 226:1322-1325.

172. McMahon NJ, Gordon HW, Rosen RB: Reed-Sternberg cells in infectious mononucleosis. Report of a case. Am J Dis Child 1970; 120:148-150

173. Mellstedt H, Holm G, Bjorkholm M: Multiple myeloma, Waldenstrorn's macroglobulinemia, and benign monoclonal gammopathy: Characteristics of the B cell clone, immunoregulatory cell populations and clinical implications. Adv Cancer Res 1984; 41:257-289.

173a. Miller G: Epstein-Barr virus in, Fields BN et al (eds): Virology. New York, Raven Press, 1985, pp 563-587. 
174. Möller G, Möller E: The concept of immunologic surveillance against neoplasia. Foreword. Transplant Rev 1976; 28:3-16.

175. Moller P, Hermann B, Moldenhaur G, et al: Defective expression of MHC Class I antigens is frequent in B-cell lymphomas of high-grade malignancy. Int $J$ Cancer 1987; 40:32-39.

176. Morgan EL, Weigle WO: Biological activities residing in the Fc region of immunoglobulin. Adv Immunol 1987; 40:61-134.

177. Nagington J, Gray J: Cyclosporin A immunosuppression, Epstein-Barr antibody and lymphoma. Letter. Lancet 1980; 1:536-537.

178. Nalesnik MA, Starzl TE, Porter KA, et al: Genotypic analyses of Cyclosporine-associated lymphoproliferations. Transplantation 1987; 43:592-593.

179. Nemerow GR, Wolfert $R$, McNaughton ME, et al: Identification and characterization of the Epstein-Barr virus receptor on human B lymphocytes and its relationship to the C3d complement receptor (CR2). J Virol 1985; $55: 347-351$

180. Nilsson K, Giovanella BC, Stehlin JS, et al: Tumorigenicity of human hematopoietic cell lines in athymic nude mice. Int $J$ Cancer 1977; 19:337344.

181. Palutke M, Schnitzer B, Mirchandani I, et al: Increased numbers of lymphocytes with single class surface immunoglobulins in reactive hyperplasia of lymphoid tissue. Am J Clin Pathol 1982; 8:316-323.

182. Pelicci P-G, Knowles DM II, Arlin ZA, et al: Multiple monoclonal B cell expansions and c-myc oncogene rearrangements in acquired immunodeficiency syndrome-related lymphoproliferative disorders. Implications for lymphomagenesis. J Exp Med 1986; 164:2049-2076.

183. Pelicci PG, Knowles DM, Dalla Favera R: Lymphoid tumors displaying rearrangements of both immunoglobulin and T cell receptor genes. $J$ Exp Med 1985; 162:1015-1024.

184. Pelloquin F, Lamelin J-P, Lenoir GM: Human B lymphocytes immortalization by Epstein-Barr virus in the presence of Cyclosporin A. In Vitro Cell Dev Biol 1986; 22:689-694.

185. Penn I, First MR: Development and incidence of cancer following cyclosporine therapy. Transplant Proc 1986; 18(Suppl 1):210-213.

186. Penn I, Hammond W, Brettschneider L, et al: Malignant lymphomas in transplantation patients. Transplant Proc 1969; 1:106-112.

187. Penn I: Malignant lymphomas in organ transplant recipients. Transplant Proc 1981; 13:736-738.

188. Penn I: Depressed immunity and the development of cancer. Clin Exp Immunol 1981; 46:459-474.

189. Penn I: The price of immunotherapy. Curr Prob Surg 1981; 18:682-751.

190. Penn I: Brief Communication: Host origin of lymphomas in organ transplant recipients. Transplantation 1979; 27:214.

191. Penn I: Tumors arising in organ transplant recipients. Adv Cancer Res 1978; 28:31-61.

192. Penn I: Malignancies associated with immunosuppressive or cytotoxic therapy. Surgery 1978; 83:492-502.

193. Penn I: Cancers following Cyclosporine therapy. Transplantation 1987 43:32-35.

194. Perry GS, Spector BD, Shuman LM, et al: The Wiskott-Aldrich syndrome in the United States and Canada (1892-1979). J Pediatr 1980; 97:72-78.

195. Prehn RT: The immune reaction as a stimulator of tumor growth. Science $1972 ; 176: 170-171$ 
196. Prehn RT: Immunosurveillance, regeneration and oncogenesis. Prog Exp Tumor Res 1971; 14:1-24.

197. Purtilo D, Harada S, Bechtold T, et al: Immunoregulatory defects and Epstein-Barr virus-associated lymphoid disorders, in Magrath IT, O'Conor GT, Ramot B (eds): Pathogenesis of Leukemias and Lymphomas: Environmental Influences, New York, Raven Press, 1984; pp 235-257.

198. Purtilo DT, Tatsumi E, Manolov G, et al: Epstein-Barr virus as an etiological agent in the pathogenesis of lymphoproliferative and aproliferative diseases in immune deficient patients. Int Rev Exp Pathol 1985; 27:113183.

199. Purtilo DT, Cassel C, Yang JPS, et al: X-linked recessive progressive combined variable immunodeficiency (Duncan's disease). Lancet 1975; 1:935940.

200. Purtilo DT: Biology of disease. Defective immune surveillance in viral carcinogenesis. Lab Invest 1984; 51:373-385.

201. Purtilo DT: Opportunistic cancers in patients with immunodeficiency syndromes. Arch Pathol Lab Med 1987; 111:1123-1129.

202. Purtilo DT: Epstein-Barr virus-associated lymphoid and epithelial lesions occurring in the life cycle, in Levine PH, Ablashi DV, Nonoyama M, et al (eds): Epstein-Barr Virus and Human Disease. Clifton NK, Humana Press, 1987, pp 3-11.

203. Purtilo DT: Epstein-Barr virus-induced oncogenesis in immunodeficient individuals. Lancet 1980; 1:300-303.

204. Purtilo DT: Malignant lymphoproliferative disease induced by EpsteinBarr virus in immunodeficient patients including X-linked, cytogenetic and familial syndromes. Cancer Genet Cytogenet 1981; 4:251-268.

205. Raab-Traub N, Flynn K: The structure of the termini of the Epstein-Barr virus as a marker of clonal cellular proliferation. Cell 1986; 47:883-889.

206. Raab-Traub N, Flynn K, Klein G: EBV DNA structure and oncogene expression in EBV-associated malignancies, in Levine PH, Ablashi DV, Nonoyama $\mathrm{M}$, et al (eds): Epstein-Barr Virus and Human Disease Clifton NJ, Humana Press, 1987, pp 423-428.

207. Reinherz EL, Haynes BF, Nadler LM, et al: Leukocyte Typing II. New York, Springer-Verlag, 1985.

208. Reinherz EL, Royer HD, Campen TJ, et al: The structure and function of the T3-Ti molecular complex on Human T lymphocytes, in Symposium on Fundamental Cancer Research, vol 38. Houston, The University of Texas System Cancer Center, 1986, pp 3-30.

209. Diffuse, undifferentiated non-Hodgkin's lymphomas among homosexual males-United States. Morbid Mortal Weekly Rep 1982; 31:277-279.

210. Rickinson $A B$ : Cellular immunologic response to the virus infection, in Epstein MA, Achong BG (eds): The Epstein-Barr Virus: Recent Advances. New York, John Wiley and Sons, 1986, pp 75-125.

211. Roberts WM, Wotherspoon R, Herrod HG, et al: Morbidity of Epstein-Barr virus infection in children, in Levine PH, Ablashi DV, Nonoyama $\mathrm{M}$ (eds): Epstein-Barr Virus and Human Disease Cilfton NJ, Humana Press, 1987, pp 101-104.

212. Robinson JE, Brown N, Andiman W, et al: Diffuse polyclonal B-cell lymphoma during primary infection with Epstein-Barr virus. $N$ Engl $J$ Med 1980; 302:1293-1297.

213. Robinson JE, Smith D, Niederman J: Plasmacytic differentiation of circulating Epstein-Barr virus-infected B lymphocytes during acute infectious mononucleosis. J Exp Med 1981; 153:235-244. 
214. Rosen A, Gergely $P$, Jondal $M$, et al: Polyclonal Ig production after EpsteinBarr virus infection of human lymphocytes in vitro. Nature 1977; 267:5254.

215. Rosenthal JT, Iwatsuki S, Starzl TE, et al: Histiocytic lymphoma in renal transplant patients receiving Cyclosporine. Transplant Proc 1983; 15:28052807.

216. Rous $P$, Beard JW: The progression to carcinoma of virus-induced rabbit papillomas (Shope). J Exp Med 1935; 62:523-548.

217. Rous P, Kidd JG: Conditional neoplasms and subthreshold neoplastic states. A study of the tar tumors of rabbits. J Exp Med 1941; 73:365-390.

218. Saemundsen AK, Purtilo DT, Sakamoto K, et al: Documentation of EpsteinBarr virus infection in immunodeficient patients with life-threatening lymphoproliferative diseases by Epstein-Barr virus complementary RNA DNA and viral DNA/DNA hybridization. Cancer Res 1981; 41:4237-4242.

219. Salvador AH, Harrison EG, Kyle RA: Lymphadenopathy due to infectious mononucleosis: Its confusion with malignant lymphoma. Cancer 1971; 27:1029-1040.

220. Sarid J, Halazonetis TD, Murphy W, et al: Evolutionarily conserved regions of the human c-myc protein can be uncoupled from transforming activity. Proc Natl Acad Sci USA 1987; 84:170-173.

221. Sato T: Acute Epstein-Barr infection and diffuse large-cell lymphoma. $J$ Infect 1985; 10:265-267.

222. Schubach $W H$, Hackman $R$, Nieman PE, et al: A monoclonal immunoblastic sarcoma in donor cells bearing Epstein-Barr virus genomes following allogeneic marrow grafting for acute lymphoblastic leukemia. Blood 1982; 60:180-187.

223. Schwartz RS: Another look at immunologic surveillance. $N$ Engl $J$ Med 1975; 293:181-184.

224. Sedmak DD, Deodhar SD, Tubbs RR: Post-transplantation reticulum cell sarcoma reclassified as B-cell lymphoma. Letter. $N$ Engl $J$ Med 1985; 312:1329-1330.

225. Shackney SE, Levine AM, Fisher RI, et al: The biology of tumor growth in the non-Hodgkin's lymphomas. A dual parameter flow cytometry study of 220 cases. $J$ Clin Invest 1984; 73:1201-1214.

226. Shapiro RS, McClain K, Blazar B, et al: EBV-related B-cell lymphoproliferative disease after $\mathrm{T}$ depleted mismatched bone marrow transplantation, in Levine PH, Ablashi DV, Nonoyama M, et al (eds): Epstein-Barr Virus and Human Disease. Clifton NJ, Humana Press, 1987, pp 91-95.

227. Shearer WT, Ritz J, Finegold MJ, et al: Epstein-Barr virus-associated B-cell proliferations of diverse clonal origins after bone marrow transplantation in a 12 year patient with severe combined immunodeficiency. $N$ Engl $J$ Med 1985; 312:1151-1159.

228. Sheibani K, Wu A, Ben-Ezra J, et al: Rapid communication: Rearrangement of $\mathrm{k}$-chain and T-cell receptor $\beta$-chain genes in malignant lymphomas of "T-cell" phenotype. Am J Pathol 1987; 129:201-207.

229. Sheil AGR, Flavel S, Disney APS, et al: Cancer in dialysis and transplant patients. Transplant Proc 1985; 17:195-198.

230. Sheil AGR, Flavel S, Disney APS, et al: Cancer development in patients progressing to dialysis and renal transplantation. Transplant Proc 1985; 17:1685-1688.

231. Sheil AGR: Cancer after transplantation. World J Surg 1986; 10:389-396.

232. Shevach EM: The effects of Cyclosporine A on the immune system. Ann Rev Immunol 1985; 3:397-423. 
233. Shope T, Dechairo D, Miller G: Malignant lymphoma in cottontop marmosets after inoculation with Epstein-Barr virus. Proc Natl Acad Sci USA 1973; 70:2487-2491.

234. Siegelman MH, Cleary ML, Warnke R, et al: Frequent biclonality and Ig gene alterations among $\mathrm{B}$ cell lymphomas that show multiple histologic forms. J Exp Med 1985; 161:850-863.

235. Simmons RL, Najarian JS: reply to Starzl TE, Penn I, Halgrimson CG: Immunosuppression and malignant neoplasms (letter). $N$ Engl $J$ Med 1970: 283:934-935.

236. Sixbey JW, Nedrud JG, Raab-Traub N, et al: Epstein-Barr virus replication in oropharyngeal epithelial cells. $N$ Engl $J$ Med 1984; 310:1225-1230.

237. Sixbey JW, Pagano JS: Epstein-Barr virus transformation of human B-lymphocytes despite inhibition of viral polymerase. J Virol 1985; 53:299-301.

238. Skare JC, Milunsky A, Byron KS, et al: Mapping the X-linked lymphoproliferative syndrome. Proc Natl Acad Sci USA 1987; 84:2015-2018.

238a. Smith HO, Wilcox KW: A restriction enzyme from Hemophilus influenza. 1. Purification and general properties. J Mol Biol 1970; 51:379-391.

239. Snider WD, Simpson DM, Aronyk KE, et al: Primary lymphoma of the nervous system associated with acquired immunodeficiency syndrome. Letter. $N$ Engl $J$ Med 1983; 308:45.

240. Southern EM: Detection of specific sequences among DNA fragments separated by gel electrophoresis. $J$ Mol Biol 1975; 98:503-517.

241. Spector BD, Filipovich AH, Perry GS, et al: Epidemiology of cancer in ataxia-telangiectasia, in BA Bridges, DG Harnden (eds): Ataxia-Telangiectasia-A Cellular and Molecular Link Between Cancer, Neuropathology, and Immune Deficiency, New York, John Wiley and Sons, 1982, pp 103-138.

242. Srigley J, Barlogie B, Butler JJ, et al: Heterogeneity of non-Hodgkin's lymphoma probed by nucleic acid cytometry. Blood 1985; 65:1090-1096.

243. Starzl TE, Klintmalm GBG, Weil R III, et al: Cyclosporine A and steroid therapy in sixty-six cadaver kidney recipients. Surg Gynecol Obstet 1981 153:486-494.

244. Starzl TE, Nalesnik MA, Porter KA, et al: Reversibility of lymphomas and lymphoproliferative lesions developing under Cyclosporine-steroid therapy. Lancet $1984 ; 1: 583-587$.

245. Starzl TE, Penn I, Halgrimson CG: Immunosuppression and malignant neoplasms. Letter. N Engl J Med 1970; 283:934.

246. Starzl TE: Discussion of Murray JE, Wilson RE, Tilney NL, et al: Five years' experience in renal transplantation with immunosuppressive drugs: Survival, function, complications and the role of lymphocyte depletion by thoracic duct fistula. Ann Surg 1968; 168:416-435.

247. Strauch B, Slegel N, Andrews L, et al: Oropharyngeal excretion of EpsteinBarr virus by renal transplant recipients and other patients treated with immunosuppressive drugs. Lancet 1974; 1:234-237.

248. Straus SE, Tosato G, Armstrong G, et al: Persisting illness and fatigue in adults with evidence of Epstein-Barr virus infection. Ann Intern Med 1985; 102:7-16.

249. Strum SB, Park JK, Rappaport H: Observation of cells resembling Sternberg-Reed cells in conditions other than Hodgkin's disease. Cancer 1970; 26:176-190.

250. Stutman O: Immunologic surveillance revisited, in AE Reif, MS Mitchell (eds): Immunity to Cancer. New York, Academic Press, 1985, pp 323-345.

251. Stutman $O$ : The Immunologic surveillance hypothesis, in RB Herberman 
(ed): Basic and Clinical Tumor Immunology. Boston, Martinus Nijhoff, 1983, pp 1-83.

252. Sullivan JL, Woda BA, Herrod HG, et al: Epstein-Barr virus-associated hemophagocytic syndrome: Virological and immunopathological studies. Blood 1985; 65:1097-1104.

253. Svedmyr E, Ernberg I, Seeley J, et al: Virologic, immunologic, and clinical observations on a patient during the incubation, acute and convalescent phases of infectious mononucleosis. Clin Immunol Immunopathol 1984; 30:437-450.

254. Tanaka A, Donovan J, Smith M, et al: NIH 3T3 cell transformation by DNAs from lymphoma cells and from Epstein-Barr virus-immortalized human lymphocytes, in Levine PH, Ablashi DV, Nonoyama M, et al (eds): EpsteinBarr Virus and Human Disease Clifton, NJ, Humana Press, 1987, pp 429433.

255. Thiru S, Calne RY, Nagington J: Lymphoma in renal allograft patients treated with Cyclosporin-A as one of the immunosuppressive agents. Transplant Proc 1981; 13:359-364.

255a. Thomas L: Discussion of paper by PB Medawar (1957). Reactions to homologous tissue antigens in relation to hypersensitivity, in Lawrence HS (ed): Cellular and Humoral aspects of the Hypersensitive States New York, Paul Hoeber Inc, 1959, p 530.

256. Thomson AW, Whiting PH, Blair JT, et al: Pathological changes developing in the rat during a 3-week course of high dosage cyclosporin A and their reversal following drug withdrawal. Transplantation 1981; 32:271-277.

257. Thomson AW, Whiting PH, Cameron ID, et al: A toxicological study in rats receiving immunotherapeutic doses of cyclosporine A. Transplantation 1981; 31:121-124.

258. Tosato G, Steinberg AD, Yarchoan R, et al: Abnormally elevated frequency of Epstein-Barr virus-infected B cells in the blood of patients with rheumatoid arthritis. $J$ Clin Invest 1984; 73:1789-1795.

259. Touraine JL, Bosi E, El Yafi MS, et al: The infectious lymphoproliferative syndrome in transplant patients under immunosuppressive therapy. Transplant Proc 1985; 17:96-98.

260. Touraine JL, El Yafi S, Bosi E, et al: Immunoglobulin abnormalities and infectious lymphoproliferative syndrome (ILPS) in Cyclosporine-treated transplant patients. Transplant Proc 1983; 15(Suppl 1):2798-2804.

261. Toyonaga B, Yoshikai Y, Vadasz V, et al: Organization and sequences of the diversity, joining, and constant region genes of the human T-cell receptor $\beta$ chain. Proc Natl Acad Sci USA 1985; 82:8624-8628.

262. Tsujimoto $Y$, Erikson J, Nowell PC, et al: Molecular genetics of human $B$ cell neoplasia, in Symposium on Fundamental Cancer Research, vol 38. Houston, University of Texas System Cancer Center, 1986, pp 79-93.

263. Tubman DE, Frick MP, Hanto DW: Lymphoma after organ transplantation: Radiologic manifestations in the central nervous system, thorax and abdomen. Radiology 1983; 149:625-631.

263a. Uhr JW: The 1984 Nobel Prize in Medicine. Science 1984; 226:1025-1028.

264. Waldmann TA, Korsmeyer SJ, Greene WC: The arrangement of immunoglobulin, $\mathrm{T}$ cell antigen receptor, and interleukin 2 receptor genes in human lymphoid neoplasms, in Symposium on Fundamental Cancer Research, vol 38. Houston, University of Texas System Cancer Center. 1986; pp 63-78.

265. Williams ME, Innes DJ, Borowitz MJ, et al: Immunoglobulin and $\mathrm{T}$ cell 
receptor gene rearrangements in human lymphoma and leukemia. Blood 1987; 69:79-86.

266. Wilson RE, Hager EB, Hampers CL, et al: Immunologic rejection of human cancer transplanted with a renal allograft. $N$ Engl J Med 1968; 278:479-483.

267. Wolf $H$, Haus M, Wilmes E: Persistence of Epstein-Barr virus in the parotid gland. $J$ Virol 1984; 51:795-798.

268. Yao QY, Rickinson $A B$, Epstein MA: A re-examination of the Epstein-Barr virus carrier state in healthy seropositive individuals. Int $J$ Cancer 1985; $35: 35-42$.

269. Yao QY, Rickinson $\mathrm{AB}$, Gaston JSH, et al: In vitro analysis of the EpsteinBarr virus: Host balance in long-term renal allograft recipients. Int $J$ Cancer 1985; 35:43-49.

270. Yunis JJ: Chromosome and oncogene rearrangements in leukemia and lymphoma, in Fenoglio-Preiser CM, Weinstein RS, Kaufman N, (ed): New Concepts in Neoplasia as Applied to Diagnostic Pathology. IAP Monograph 27. Baltimore, Williams and Wilkins, 1986, pp 91-96.

271. Ziegler JL, Levy JA: Acquired immunodeficiency syndrome and cancer. Adv Viral Oncol 1985; 5:239-255.

272. Ziegler JL, Beckstead JA, Volberding PA, et al: Non-Hodgkin's lymphoma in 90 homosexual men. Relation to generalized lymphadenopathy and the acquired immunodeficiency syndrome. $N$ Engl J Med 1984; 311:565-570.

273. Zinkernagel R, Doherty P: MHC-restricted cytotoxic T cells: Studies on the biological role of polymorphic major transplantation antigens determining $\mathrm{T}$ cell restriction-specificity, function and responsiveness. Adv Immunol 1979; 27:51-177.

274. Zukoski CF, Killen DA, Ginn E, et al: Transplanted carcinoma in an immunosuppressed patient. Transplantation 1970; 9:71-74. 